\title{
Networks in Production: Asset Pricing Implications *
}

\author{
Bernard Herskovic ${ }^{\dagger}$ \\ Job Market Paper $\ddagger$
}

January 22, 2015

\begin{abstract}
This paper studies asset pricing in a multisector model in which sectors are connected to each other through an input-output network. Changes in the structure of the network are sources of systematic risk reflected in equilibrium asset prices. There are two key characteristics of the network that matter for asset prices: network concentration and network sparsity. Network concentration measures the degree to which equilibrium output is dominated by few large sectors while network sparsity measures the average input specialization of the economy. Furthermore, these two productionbased asset pricing factors are determined by the structure of the network of production and can be computed from input-output data. By sorting stocks based on their exposure to the network factors, I find a return spread of $6 \%$ per year on portfolios sorted on sparsity-beta and $-4 \%$ per year on portfolios sorted on concentration-beta. These return gaps cannot be explained by standard asset pricing models such as the CAPM or the Fama French three-factor model. A calibrated model matches the network factor betas and return spreads alongside other asset pricing moments.
\end{abstract}

Keywords: Networks, Input-Output, Systematic Risk.

JEL Codes: G12, G11, E13, E16.

*I am extremely grateful to Stijn van Nieuwerburgh for his invaluable support and input to this project. I also want to thank Alberto Bisin and Boyan Jovanovic for their numerous comments and suggestions. I would like to thank Edouard Schaal, Gianluca Violante, Jaroslav Borovička, Katarína Borovička, Alireza TahbazSalehi, Stanley E. Zin, David Backus, Jess Benhabib, Viral Acharya, Xavier Gabaix, Cecilia Parlatore, Alexi Savov Itamar Drechsler, Theresa Kuchler, Johannes Stroebel, Eduardo Davila, João Ramos, Joseph Briggs, Vadim Elenev, Ross Doppelt, Elliot Lipnowski, and participants at the NYU Stern Macro Lunch Seminar, NYU Macro Student Lunch Seminar, NYU Financial Economics Workshop, and NYU Stern Finance Job Market Workshop.

${ }^{\dagger}$ Email: herskovic@nyu.edu.

†Updates: https://files.nyu.edu/bh922/public/BernardHerskovicJMP.pdf 


\section{Introduction}

Firms use a variety of inputs to build their own products, collectively spending trillions of dollars and constituting a network of input-output linkages. As technology evolves, industries may use different inputs to produce their final output. For example, since the 1970s, plastics have become a more suitable substitute for wood and metal materials, reshaping the production process for manufacturing and construction. Changes in the input-output network have implications for the overall economy as they alter sectoral linkages. In this paper, I investigate the implications of changes in the input-output network for asset prices and aggregate quantities such as consumption and GDP. I show that changes in the network are a source of systematic risk that is priced in equilibrium. To the best of my knowledge, this paper is the first to explore the asset pricing implications of a sectoral network model.

The main result of this paper is that there are two key network factors that matter for asset prices: network concentration and network sparsity. The network factors are characteristics that describe specific attributes of sectoral linkages. I demonstrate that concentration and sparsity constitute sufficient statistics for aggregate risk. Although the entire inputoutput linkage network is multidimensional, we may focus on these two characteristics when assessing systematic risk. I derive the network factors from a general equilibrium model, and these factors determine the dynamics of aggregate output and consumption. Moreover, innovations in concentration and sparsity may be computed from the data and empirically tested as new asset pricing factors. Return data shows that exposure to these network factors is reflected in average returns as predicted by my model.

Network concentration measures how concentrated sectors' output shares are in equilibrium. Sectors' equilibrium output shares represent how important the output of a particular sector is to all other sectors as a source of input. If the output of a sector is widely used as input by other sectors, then it has high output share in equilibrium. Whether a sector has high or low output share depends on the network and therefore concentration is an attribute of the network.

Network sparsity is a characteristic of the sectoral linkages distribution. Sectoral linkages are directly related to how important each input is to a particular sector, and sparsity measures the degree of input specialization of the economy and how crowded or dense these linkages are in the network. A network with high sparsity has fewer linkages, but these linkages are stronger and, on average, firms rely on fewer sources of input.

Empirically, input-output data from the BEA provides a picture of the production net-

work of the U.S. economy. Figure 1 plots the network representation of the input-output linkages, where nodes (circles) represent different sectors and edges (arrows) represent input 
Figure 1: Input-Output Network Representation at the Sector Level

This picture contains the Network representation of the BEA Input-Output data for 2012 at the sector level (two-digit NAICS). An arrow from sector $j$ to sector $i$ means that $j$ is selling to $i$; the intensity of the arrow (transparency and width) captures how much $i$ is buying from $j$ relative to other suppliers. The label in each node is the 2-digit NAICS sector. The size of a node (sector) represents the output share; the diameter of a node is proportional to the output share.

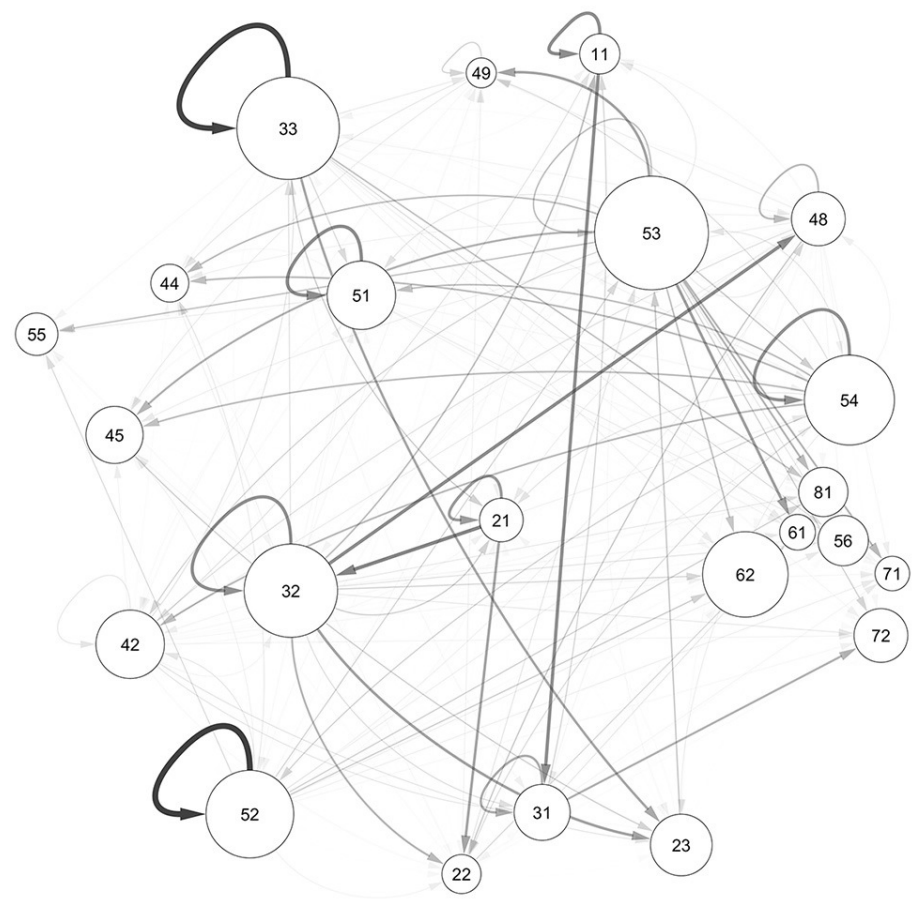

flow between sectors. An arrow from sector $j$ to sector $i$ illustrates the input flow from sector $j$ to sector $i$. The size of a node represents the sector's output share, and the thickness of an edge represents the input expenditure share. Concentration captures the degree to which output is dominated by few sectors, and it is measured by the concentration over node sizes. If there are a few large nodes (sectors with large output share) as the graph illustrates to be the case for the U.S. economy, then concentration is greater than in a economy in which nodes had the same size. Sparsity captures the degree of input specialization and thus measures how thick and scarce network edges are. An economy with high sparsity and therefore high input specialization has fewer edges, but these edges are thicker. Hence, concentration is a characteristic of the nodes' size distribution whereas sparsity is a characteristic of the edges' thickness distribution.

When production is subject to diminishing returns, an economy with high concentration has few large sectors with lower return to investments. The lower productivity of large sectors affects other sectors through equilibrium prices. As a result, high concentration leads to lower aggregate consumption and higher marginal utility. Thus innovations in concentration 
carry a negative price of risk. Assets that have high returns when concentration increases, i.e. assets with high concentration-beta, are hedges against drops in aggregate consumption, and they should have lower expected returns. A portfolio that goes long high concentration-beta stocks and short low concentration-beta stocks should have negative average returns.

Sparsity is directly related to productivity gains due to sectors' connectivity. In my model, firms have a Cobb-Douglas production technology. They use each others' inputs in order to produce their own final output, and the network specifies the importance of each input to the final output! For each sector, the network defines the elasticity of its output with respect to each input as well as the marginal product of inputs. Therefore, the network defines the shape of the production function.

When sparsity increases, firms reoptimize inputs based on the marginal product, substituting inputs that had their marginal product decreased for inputs that had their marginal product increased. The updated input allocation has two immediate implications for the final output of the firms. On the one hand, firms gain efficiency from using more inputs with higher marginal product and increase final output. On the other hand, firms substitute inputs at their relative spot market prices, changing input combinations and marginal cost of production. After sparsity increases, a particular firm may use inputs that are relatively more (less) expensive, causing the marginal cost of production to increase (decrease) and its final output to decrease (increase). Therefore, changes in the marginal cost may have positive or negative effect on output depending both on the spot market prices and on the specific changes in the network. The efficiency gain, however, always increases output. The aggregate effect from an increase in sparsity on the output of the firm depends on which effect dominates.

When concentration is kept constant, then changes in marginal cost due to different input combinations aggregate to zero. This is because some firms use inputs that are relatively more expensive and others use inputs that are relatively less expensive. Thus, aggregate output and consumption increase when the sparsity factor increases. When sparsity increases, the input-output linkages are rearranged, increasing aggregate consumption and decreasing marginal utility. Innovations in network sparsity carry positive price of risk. Assets that have high returns when network sparsity increases, i.e. asset with high sparsity-beta, are risky assets and their expected returns should be higher to compensate the investor for this risk. A portfolio that goes long high sparsity-beta stocks and short low sparsity-beta stocks should have positive average returns.

In order to illustrate the difference between the two network factors, Figure 2 plots

\footnotetext{
${ }^{1}$ For the remainder of the paper, the words 'firm' and 'sector' are used interchangeably. In my model, each sector features a representative firm.
} 
Figure 2: Changes in Network Sparsity and Network Concentration

These are are three simulated networks with 23 sectors. Panel (a) presents a network with low network sparsity factor and low network concentration, panel (b) presents a network with low network concentration, but high network sparsity. Panel (c) present a network with high sparsity and high concentration.

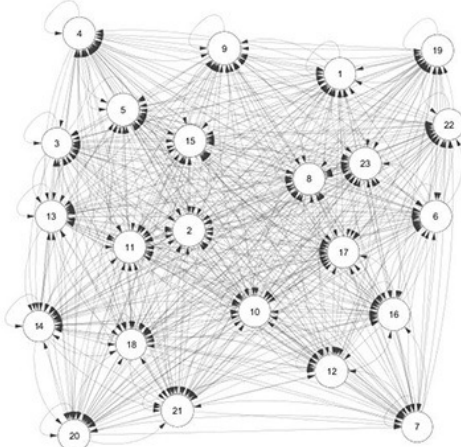

(a) Low Sparsity and Low Concentration

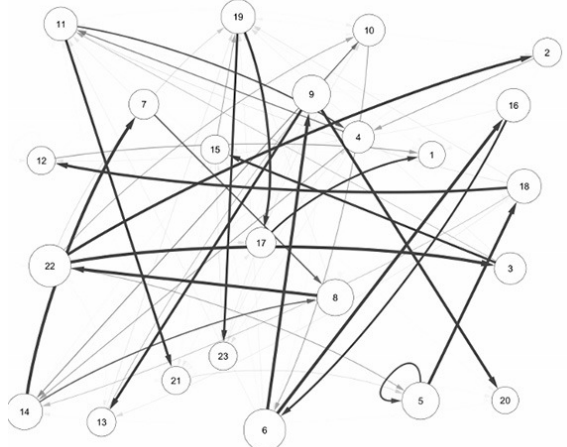

(b) High Sparsity and Low Concentration

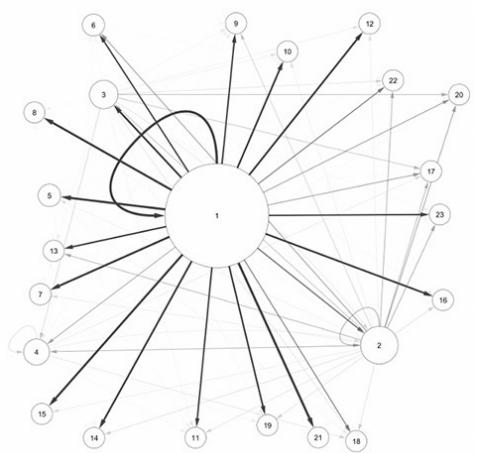

(c) High Sparsity and High Concentration

three simulated networks with different network moments in panels (a), (b) and (c). The network in panel (a) has uniform edges meaning that sectors' input expenditures are evenly distributed across inputs. Similarly, the nodes are of similar size meaning that output shares are roughly the same. This network has low sparsity and low concentration factors. The network in panel (b) has fewer edges, but thicker ones. Each sector has its input expenditure concentrated on a few sectors. As a result, the network in panel (b) has higher sparsity than the network in panel (a). Although the network in panel (b) is more sparse than in (a), concentration is the same in both networks. The network in panel (c) presents an increase in the concentration factor. The input expenditure of all other sectors is highly concentrated on sector 1 , which results in a higher output share for sector 1 and a lower share for the other sectors in equilibrium. As a result, the network in (c) has a higher concentration factor than (a) and (b). However, the edges of networks in panels (b) and (c) are just as scattered and the degree of input specialization is the same, meaning that sparsity is the same in both networks.

In addition to a time-varying network, the model features an aggregate productivity factor, a common feature of production-based models. However, in the model, this productivity factor arises endogenously from aggregating sector-specific productivity shocks. The network structure governs the extent to which these productivity shocks are diversifiable and how they generate systematic risk. Therefore, the general equilibrium model boils down to a three factor model: aggregate productivity, network concentration, and network sparsity. These three factors fully determine the dynamics of aggregate output and consumption in 
equilibrium, and innovations in concentration and sparsity represent two new candidate asset pricing factors I take to the data.

I test empirically whether high sparsity-beta assets have higher expected returns than those with low sparsity-beta, and whether high concentration-beta assets have lower expected returns than assets with low concentration-beta. The network factors are computed from Compustat, and CRSP stocks are sorted into portfolios based on their exposures to the innovations in the network factors. I sort portfolios and find that the high sparsity-beta portfolio has higher returns than the low sparsity-beta portfolio with a return difference of $6 \%$ per year. Furthermore, the high concentration-beta portfolio has lower returns than the low concentration-beta portfolio with a spread of $4 \%$ per year. These return spreads are economically meaningful and statistically significant. Moreover, neither the CAPM nor the Fama French three-factor model can explain these returns differences.

In addition to verifying beta-sorted portfolios return spreads, I show that factor-mimicking portfolios for the network sparsity and concentration factors help price other sets of equity in a Fama MacBeth analysis. Long-short sparsity-beta and long-short concentration-beta portfolios help price portfolios sorted by book-to-market ratio, by industry, and by idiosyncratic volatility level.

Finally, I investigate whether the empirical return spreads are quantitatively consistent with my model. The model is calibrated to match the return betas estimated from the data as well as other asset pricing moments, including the equity risk premium on the market portfolio, the market return volatility, and the risk-free rate of return. Importantly, the calibration respects the observed time series properties of the network factors. The calibrated model is successful in terms of replicating the average excess return of the sorted portfolios as well as their return volatility.

The rest of paper is organized as follows. The next section discusses the related literature. Section 2 presents the model and discusses the network factors. Section 3 discusses the empirical evidence, and Section 4 shows the calibrated model. Section 5 concludes.

\section{Related Literature}

The literature that applies network theory to macroeconomics and finance has mostly focused on documenting stylized facts, and building micro foundation for business cycles, financial contagion, and other macroeconomic phenomena. $2^{2}$ Asset pricing implications of sectoral

\footnotetext{
${ }^{2}$ There are several recent papers on networks and finance. The main contributions include Hou and Robinson (2006), Ahern and Harford (2010). Cohen, Frazzini, and Malloy (2008), Cohen and Frazzini (2008), Carvalho (2010), Acemoglu, Ozdaglar, and Tahbaz-Salehi (2013), Aobdia, Caskey, and Ozel (2013), Babus (2013), Biggio and La'O (2013), Carvalho and Gabaix (2013), Carvalho and Grassi (2014), Carvalho
} 
linkages, however, have been largely neglected. This paper contributes to a recent but growing literature that studies firms' customer-supplier linkages and asset prices. I extend this literature by providing new asset pricing factors constructed from the input-output network.

Using input-output data, Ahern (2012) shows that industries occupying a more central position in the network earn higher returns on average. Centrality of a particular industry is a property of a node (sector) in the network as opposed to the property of the entire network. In my model, sparsity and concentration factors are properties of the whole network. Another related paper is Kelly, Lustig, and Van Nieuwerburgh (2013) who investigate the relation between firm size distribution and firm-level volatility through the lens of a customer-supplier network model. However, they do not investigate the asset pricing implications of customer-supplier linkages. Herskovic, Kelly, Lustig, and Van Nieuwerburgh (2014) document a common factor structure in the idiosyncratic firm-level return volatility and show that the common idiosyncratic volatility factor is priced. Unlike these papers, my model derives network factors from a general equilibrium model where these factors originate from sectoral linkages and are source of systematic risk. An interesting question for future research is what the connection is between my network factors and innovations in the firm size dispersion.

This paper is also closely related to the literature that studies the importance of sectoral shocks for economic aggregates. The multisector model developed in this paper is based on Long and Plosser (1983). Their model generates comovement of sectors' output, because each sector relies on the output of other sectors as sources of inputs. My model, however, does not have the same degree of comovement, because the production technology represented by the network changes over time and therefore the sectoral shares also change over time. My model is also closely related to the work of Acemoglu, Carvalho, Ozdaglar, and Tahbaz-Salehi (2012). They show that aggregate fluctuations can be generated from sectoral idiosyncratic shocks when sectors are connected by input-output linkages $3^{3}$ The network in my model, however, changes over time, while theirs is static. Therefore, sparsity and concentration factors are absent in their analysis. Also, their paper focuses on the origins of aggregate fluctuations, while I am interested in identifying priced sources of systematic risk from changes in the network. 4

and Voigtlander (2014), Carvalho (2014), Farboodi (2014), Malamud and Rostek (2014), Finally, Allen and Babus (2008) present a detailed review of network models applied to finance.

${ }^{3}$ The idea of having aggregate shocks originate from idiosyncratic shock is also discussed by Jovanovic (1987), Bak, Chen, Scheinkman, and Woodford (1993), and Gabaix (2011).

${ }^{4}$ Carvalho (2010) presents a dynamic version of the model in Acemoglu, Carvalho, Ozdaglar, and TahbazSalehi (2012), but the network itself is fixed over time. 
This paper also contributes to the production based asset pricing literature by providing explicit asset pricing factors computed directly from the input-output network.5 The closest papers in this literature are Papanikolaou (2011), who studies how investment shocks are priced, and Loualiche (2012), who investigate aggregate entry cost as a priced risk factor. Although my model has neither entry cost nor investment shocks; both concentration and sparsity factors are related to changes in how much firms are producing in the aggregate. Changes in the network reflect not only changes in the sectoral relations, but also changes in investment opportunities. However, changes in the network factors are due to technological rearrangements that reshape the input-output network, which is different from changes in the cost producing new capital. This paper relates to a line of research that studies how technological innovation is priced. Kung and Schmid (2011) study asset pricing in a general equilibrium framework with endogenous technological growth. In my model, changes in sparsity and concentration can be interpreted as reflecting technological innovation and therefore my network factors capture two distinct risk factors resulted from changes in technology.

This paper also sheds some light on the literature on network formation. Oberfield (2013) develops an input-output network formation model. In his model, firms choose from whom they buy their inputs and the network is endogenous ${ }^{6}$ In my model, the network formation is exogenous, and the network evolves stochastically over time. An interesting question for future research is how my network factors behave in a endogenous network formation model.

\section{Multisector Network Model}

\subsection{Setup}

Time is discrete and indexed by $t=1,2, \ldots$. There are $n$ distinct goods and $n$ sectors. Each sector has one representative firm producing the good of that particular sector. For example, firm $i$ buys inputs from other sectors, and these inputs combined are transformed into the final output of sector $i$. Firms buy inputs and produce at the same time, that is, firm $i$ buys

\footnotetext{
${ }^{5}$ Related work in production-based asset pricing includes Jermann (1998, 2010, 2013) Yogo (2006), van Binsbergen (2007), Gomes, Kogan, and Yogo (2009), Kuehn (2009), Lochstoer (2009), Belo (2010), Gomes and Schmid (2010), Kuehn and Schmid (2011), Kogan, Papanikolaou, and Stoffman (2013).

${ }^{6}$ One interesting result is the existence of "star suppliers," i.e. suppliers who are simultaneously used by many other firms, as an endogenous outcome of his model. There is a recent set of studies in which endogenous network formation results in a network with a core-periphery structure when agents choose their connections unilaterally (Bala and Goyal 2000, Galeotti and Goyal 2010). Herskovic and Ramos (2014) show that, under general conditions, a hierarchical network structure emerges endogenously in a network formation game.
} 
inputs from other sectors at period $t$ and produces at period period $t$ as well: $]^{7}$ The model also features a representative household with Epstein-Zin recursive preference that owns all firms and lives off their dividends. Next, I describe the problem of the firms and how they connect to each other through input-output linkages. Then, I present the representative household problem as well as all market clearing conditions.

Firms Let's consider the maximization problem of firm $i$, and let the input bought from firm $j$ at period $t$ be denoted by $y_{i j, t}$. All inputs acquired from other firms are combined and transformed into a single investment variable given by

$$
I_{i, t}=\left[\sum_{j=1}^{n} w_{i j, t} y_{i j, t}^{1-1 / \nu}\right]^{\frac{1}{1-1 / \nu}}
$$

where $\nu$ is the elasticity of substitution between inputs and $w_{i j, t}$ the weight on, or the importance of, input $j$. The weights $w_{i j, t}$ are non-negative and sum to one, that is,

$$
w_{i j, t} \geq 0 \quad \text { and } \quad \sum_{j=1}^{n} w_{i j, t}=1 .
$$

The investment variable $I_{i, t}$ is further transformed into the final output of sector $i$ according to

$$
Y_{i, t}=\varepsilon_{i, t} I_{i, t}^{\eta}
$$

where $\eta<1$ captures decreasing returns to input investments, and $\varepsilon_{i, t}$ represents sectorspecific productivity level $:^{8}$

Although firms maximize all future discounted dividends, their optimization problem is time-separable, and it is sufficient to maximize per-period profits. Firm $i$ chooses how much to investment and which inputs to acquire in order to maximize profits, taking both the spot market prices and the input weights as given:

$$
D_{i, t}=\max _{\left\{y_{i j, t}\right\}_{j}, I_{i, t}} P_{i, t} Y_{i, t}-\sum_{j=1}^{n} P_{j, t} y_{i j, t}
$$

\footnotetext{
${ }^{7}$ The production side is based on Long and Plosser (1983), but the time dimension is collapsed: firms buy inputs and produce at the same time. Same modeling approach is used by Acemoglu, Carvalho, Ozdaglar, and Tahbaz-Salehi (2012).

${ }^{8}$ The decreasing returns to scale is interpreted as return to scale to capital, $I_{i, t}$, and each sector faces an inelastic labor (or land) supply, $L_{i, t}=1$ for every $i$ and $t$. Thus, the output function could be stated as $Y_{i, t}=\varepsilon_{i, t} I_{i, t}^{\eta} L_{i, t}^{1-\eta}=\varepsilon_{i, t} I_{i, t}^{\eta} 1^{1-\eta}$. Under this interpretation, the profit of the firms are exactly equal to the wage (rent) payment to the representative household who owns the entire labor (land) supply.
} 
subject to equations 1 and 2 , where $P_{i, t}$ is the spot market price of good $i$.

The cum-dividend value of firm $i$, denoted by $V_{i, t}$, is defined recursively by

$$
V_{i, t}=D_{i, t}+\mathbb{E}_{t}\left[M_{t+1} V_{i, t+1}\right]
$$

where $M_{t+1}$ is the stochastic discount factor that prices all assets in the economy.

Network The network consists of all weights $w_{i j, t}$, which are taken as given by the firms max maximizing profits. Formally, the network is characterized by the following $n \times n$ matrix:

$$
W_{t} \equiv\left(\begin{array}{ccc}
w_{11, t} & \ldots & w_{1 n, t} \\
\vdots & \ddots & \vdots \\
w_{n 1, t} & \ldots & w_{n n, t}
\end{array}\right)
$$

The network represents how firms' production is interconnected. It informs how much a firm my influence or be influenced by other firms. Furthermore, the network defines the production technology through equation 1. When $\nu=1$, the investment equation 1 becomes a Cobb-Douglas function, and a network weight $w_{i j, t}$ becomes the elasticity of the investment of sector $i$ with respect to input $j$. Therefore, $w_{i j, t}$ is informative about the responsiveness of output $i$ regarding changes in the amount of input $j$ used. The network and the productivity shocks evolve over time according to an stochastic process known to all agents.

Representative household The representative household has Epstein-Zin recursive references with respect to a consumption aggregator:

$$
U_{t}=\left[(1-\beta) \mathcal{C}_{t}^{1-\rho}+\beta\left(\mathbb{E}_{t}\left(U_{t+1}^{1-\gamma}\right)\right)^{\frac{1-\rho}{1-\gamma}}\right]^{\frac{1}{1-\rho}},
$$

where $\gamma$ is risk aversion, $\rho$ is the inverse of the elasticity of intertemporal substitution and $\mathcal{C}_{t}$ is a consumption aggregator.

The consumption aggregator is Cobb-Douglas and given by

$$
\mathcal{C}_{t}=\prod_{i=1}^{n} c_{i, t}^{\alpha_{i}}
$$

where $c_{i, t}$ is the consumption of good $i$ at period period $t$, and $\alpha_{i}$ is the preference weight on good $i$. The preferences weights are assumed to be constant over time and they sum to one. 
The household budget constraint is given by

$$
\sum_{i=1}^{n} P_{i, t} c_{i, t}+\sum_{i=1}^{n}\left(V_{i, t}-D_{i, t}\right) \varphi_{i, t+1}=\sum_{i=1}^{n} V_{i, t} \varphi_{i, t}
$$

where $V_{i, t}$ is the cum-dividend value of firm $i$ at period $t, \varphi_{i, t}$ is the ownership of firm $i$ at period $t$, and $D_{i, t}$ is the dividend paid at period $t$ by firm $i$. In the budget constraint, total expenditure in consumption goods and firms' shares net of dividends (left-hand side) must equal shares' value (right-hand side).

In each period, the representative agent chooses how much to consume of each good, $\left\{c_{i, t}\right\}_{i}$, and next period firms' ownership, $\left\{\varphi_{i, t+1}\right\}_{i}$ in order to maximize the recursive utility given by equation 3. The household cannot store goods from one period to another and therefore cannot save. One could assume that there is a risk-free asset in zero net supply, but in equilibrium the household has to have a zero net position to satisfy clearing conditions, which wouldn't change his consumption allocation rule.

The household problem may be stated as:

$$
J_{t}\left(P_{t}, \varphi_{t}, Q_{t}, h_{t}\right)=\max _{\left\{c_{i, t} \varphi_{i, t+1}\right\}_{i}}\left[(1-\beta) \mathcal{C}_{t}^{1-\rho}+\beta\left(\mathbb{E}_{t}\left(J_{t+1}^{1-\gamma}\right)\right)^{\frac{1-\rho}{1-\gamma}}\right]^{\frac{1}{1-\rho}}
$$

subject to equation 4 .

Market clearing There are two sets of market clearing conditions. First, all good markets clear,

$$
c_{i, t}+\sum_{j=1}^{n} y_{j i, t}=Y_{i, t} \quad \forall i, t
$$

where $c_{i, t}$ is the household consumption of $\operatorname{good} i, \sum_{j=1}^{n} y_{j i, t}$ is the total demand for good $i$ as source of input in the economy, and $Y_{i, t}$ is the total supply of good $i$.

Second, all asset markets clear,

$$
\varphi_{i, t}=1 \quad \forall i, t
$$

and the household owns all firms. The household is a representative shareholder as well.

\subsection{Competitive equilibrium}

Definition. A competitive equilibrium consists of spot market prices $\left(P_{1, t}, \cdots, P_{n, t}\right)$, consumption bundle $\left(c_{1, t}, \cdots, c_{n, t}\right)$, shares holdings $\left(\varphi_{1, t}, \cdots, \varphi_{n, t}\right)$ and inputs bundles $\left(y_{i j, t}\right)_{i j}$ 
such that, for every period $t$, (i) household and firms optimize, taking the network and spot market prices as given, and (ii) market clearing conditions 5 and 6 hold.

In order to solve the multisector model for the competitive equilibrium, we have to define the agents' optimality conditions. On the production side, the first order conditions of firm $i$ are given by

$$
\begin{aligned}
y_{i j, t} & =\mu_{i, t}^{\nu} \frac{w_{i j}^{\nu} I_{i, t}}{P_{j, t}^{\nu}} \\
I_{i, t} & =\left(\frac{\eta P_{i, t} \varepsilon_{i, t}}{\mu_{i, t}}\right)^{\frac{1}{1-\eta}}, \\
\mu_{i, t} & =\left[\sum_{j=1}^{n} w_{i j}^{\nu} P_{j, t}^{1-\nu}\right]^{\frac{1}{1-\nu}},
\end{aligned}
$$

where $\mu_{i, t}$ is a network-weighted average of spot market prices and is the shadow price of investment $-\mu_{i, t}$ is the Lagrange multiplier on the $I_{i, t}$ constraint 1. Equation 7 specifies the optimal input allocation for a given investment and equation 8 pins down the investment level itself. Detailed derivations are provided in Appendix $\mathrm{A}$.

For the household, the intra-period consumption rule is given by

$$
c_{i, t}=\alpha_{i} \frac{\sum_{j=1}^{n} D_{j, t}}{P_{i, t}},
$$

which is a direct implication of the Cobb-Douglas consumption aggregator which implies that the household spends a share $\alpha_{i}$ of her income on good $i$. The first order condition for the inter-temporal consumption allocation problem yields

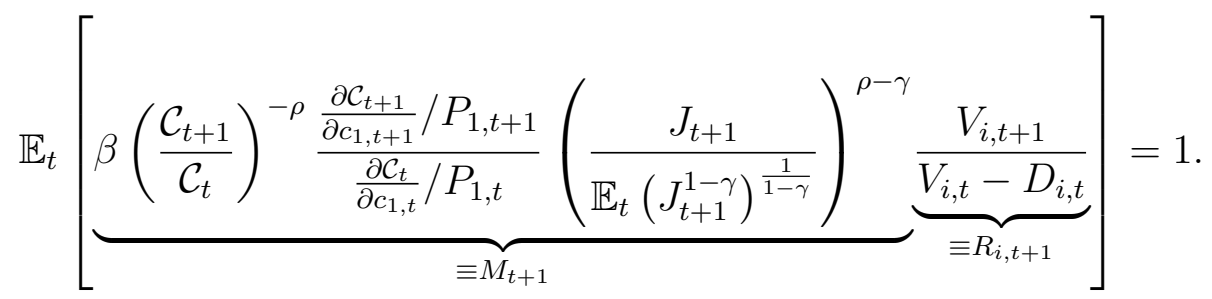

This is the Epstein-Zin first order condition for investing in firm $i$, where $M_{t+1}$ is the stochastic discount factor and $R_{i, t+1}$ is the one-period return of holding firm $i$ 's share from $t$ to $t+1$. The household choose assets' holdings, $\left\{\varphi_{i, t+1}\right\}_{i}$, such that equation 11 holds for every asset $i$. 
Therefore, the competitive equilibrium is fully determined by the optimally conditions of the firms (equations 7, 8 and 9), the household first order conditions (equations 10 and 11), and all market clearing conditions (equations 5 and 60. In addition, spot market prices may be normalized.

When spot market prices satisfy

$$
\prod_{j=1}^{n} P_{j, t}^{\alpha_{j}}=\prod_{j=1}^{n} \alpha_{j}^{\alpha_{j}} \forall t
$$

the consumption aggregator becomes the numeraire of the economy, and the utility aggregator equals the household consumption expenditure, $\mathcal{C}_{t}=\sum_{i=1}^{n} P_{i, t} c_{i, t}{ }^{9}$ The price normalization is not only useful to interpret the numeraire of the economy, but also to simplify the pricing kernel of the assets. Under this price normalization, the marginal aggregator term in the stochastic discount factor equals 1 , that is, $\frac{1}{P_{1, t}} \partial \mathcal{C}_{t} / \partial c_{1, t}=1$ for every $t$. Thus, the normalization considerably simplifies the expression for the stochastic discount factor. Lemma 1 shows that it may be written in terms of the consumption expenditure growth and the return on total wealth. This is a standard result of Epstein-Zin preferences with a slight generalization for a consumption aggregator whenever homogeneous of degree one. The detailed proof of the lemma is provided in Appendix C.

Lemma 1. If the consumption aggregator is homogeneous of degree one, then the SDF can be written as

$$
M_{t+1}=\beta^{\theta}\left(\frac{\frac{1}{P_{1, t+1}} \partial \mathcal{C}_{t+1} / \partial c_{1, t+1}}{\frac{1}{P_{1, t}} \partial \mathcal{C}_{t} / \partial c_{1, t}}\right)^{1-\gamma}\left(\frac{\omega_{t+1}}{\omega_{t}}\right)^{-\rho \theta}\left(R_{t+1}^{\mathcal{W}}\right)^{\theta-1}
$$

where $R_{t+1}^{\mathcal{W}}=\frac{\mathcal{W}_{t+1}}{\mathcal{W}_{t}-\omega_{t}}$ is return on total wealth, $\omega_{t}=\sum_{i=1}^{n} P_{i, t} c_{i, t}$ is period t total expenditure on consumption goods, and $\theta=\frac{1-\gamma}{1-\rho}$. Furthermore, when the price normalization from equation 12 holds, then

$$
M_{t+1}=\beta^{\theta}\left(\frac{\mathcal{C}_{t+1}}{\mathcal{C}_{t}}\right)^{-\rho \theta}\left(R_{t+1}^{\mathcal{W}}\right)^{\theta-1}
$$

\subsection{Closed-form expressions}

In this section, I develop closed-form expressions for output shares and consumption expenditure growth 10

\footnotetext{
${ }^{9}$ See Appendix $\mathrm{B}$ for detailed discussion and derivation.

${ }^{10}$ The detailed derivations are in Appendix D.
} 
Output shares The solution to the system of market clearing conditions 5 determines equilibrium output shares as a function of the network and preferences parameters alone, whenever the elasticity of substitution between inputs, $\nu$, equals one.

When $\nu \neq 1$, the equilibrium output shares are given by

$$
\delta_{t}=(1-\eta)\left[\mathbb{I}-\eta \tilde{W}_{t}^{\prime}\right]^{-1} \alpha,
$$

where $\delta_{t}=\left(\delta_{1, t}, \ldots, \delta_{n, t}\right)^{\prime}$ is a $n \times 1$ vector of output shares, $\tilde{W}_{t}^{\prime}$ is a $n \times n$ matrix with $(i, j)$ entry given by $\tilde{w}_{i j, t}=\frac{w_{i j, t}^{\nu} P_{j, t}^{1-\nu}}{\sum_{s} w_{i s}^{\nu} P_{s}^{1-\nu}}$, and $\alpha=\left(\alpha_{1}, \ldots, \alpha_{n}\right)^{\prime}$ is a $n \times 1$ vector of preference weights 11

When $\nu=1$, we have that $\tilde{w}_{i j, t}=w_{i j, t}$, and the output shares is completely determined by the network and household preferences. In fact, for the Cobb-Douglas case, the output shares are equal to the network centrality of the firm, a measure developed by Katz (1953). The Katz centrality quantifies the relative importance of each node in a network, that is, the relative importance of each firm to the aggregate economy. Furthermore, this measure captures indirect effects that each sector has on each other,

$$
\delta_{t}=(1-\eta)\left[\mathrm{I}-\eta W_{t}^{\prime}\right]^{-1} \alpha=(1-\eta)\left[\mathrm{I}+\eta W_{t}^{\prime}+\eta^{2} W_{t}^{\prime 2}+\eta^{3} W_{t}^{\prime 3}+\ldots\right] \alpha,
$$

where the return to scale parameter is the decaying rate of these feedback effects.

The output share of firm $j$ may be defined recursively and decomposed in two parts, a preference component and a network component:

$$
\delta_{j, t}=\underbrace{(1-\eta) \alpha_{j}}_{\text {preference component }}+\underbrace{\eta \sum_{i=1}^{n} w_{i j, t} \delta_{i, t}}_{\text {network component }}
$$

The preference component represents the household demand for goods from sector $j$ directly, and the network component captures the demand for good $j$ when used as input. The specific contribution of sector $i$ to $j$ 's output share depends on sector $i$ own share, $\delta_{i, t}$, and on the network weight connecting both sectors, $w_{i j, t}$.

We may iterate the recursive expression of output shares in order to obtain an represen-

\footnotetext{
${ }^{11}$ The output share derivation is similar to the one in Acemoglu, Carvalho, Ozdaglar, and Tahbaz-Salehi (2012). My derivation, however, is for a general CES production function and for a consumption aggregator with different preference weights.
} 
tation capturing all indirect effects along the network linkages:

$$
\delta_{j, t}=\underbrace{(1-\eta) \alpha_{j}}_{\text {preference component }}+\underbrace{\eta\left[\sum_{i=1}^{n} \alpha_{i} w_{i j, t}+\eta \sum_{i=1}^{n} \sum_{k=1}^{n} \alpha_{i} w_{i k, t} w_{k j, t}+\eta^{2} \sum_{i=1}^{n} \sum_{k=1}^{n} \sum_{s=1}^{n} \alpha_{i} w_{i k, t} w_{k s, t} w_{s, j, t}+\ldots\right]}_{\text {network component }} .
$$

The first term of the network component captures the importance of firm $j$ to its immediate customers, firms directly connected to $j$. The second term captures the indirect importance of $j$ through firms that buy inputs from $j$ own customers, that is to say, the customers of the costumers of firm $j$. The third term captures the importance of $j$ through customers that are even further away, two customers away from $j$ to be precise. All these indirect effects decay at the rate given by the returns to scale $\eta$. As firms increase production the marginal product decreases and the demand of a particular customer has a decaying effect along the production chain.

Consumption growth The stochastic discount factor, however, depends on changes in the $\log$ consumption aggregator, $\log \left(\frac{\mathcal{C}_{t+1}}{\mathcal{C}_{t}}\right)$, according to lemma 1 . Changes in the $\log$ consumption aggregator are identical to changes in the log aggragate output:

$$
\log \left(\frac{\mathcal{C}_{t+1}}{\mathcal{C}_{t}}\right)=\log \left(\frac{z_{t+1}}{z_{t}}\right)
$$

where $z_{t}=\sum_{i=1}^{n} P_{i, t} Y_{i, t}$ is aggregate output. The above equality holds because the consumption aggregator is proportional to the the aggregate output:

$$
\mathcal{C}_{t}=\sum_{j} P_{i, t} c_{i, t}=\sum_{j} D_{j, t}=(1-\eta) \sum_{j} P_{j, t} Y_{j, t}=(1-\eta) z_{t}
$$

The first equality holds as the consumption aggregator equals consumption expenditure when price normalization 12 is satisfied. The second equality comes from the budget constraint and the clearing conditions combined. The third one is based on the firms' optimality conditions, and the last uses the aggregate output definition.

The aggregate output is part of the solution of all market clearing conditions and agents' first order condition. The household consumption has already been solved in closed form by equation 10, and the output shares given by expression 14 satisfy the market the clearing conditions 5. To solve the model for the aggregate output, we have to solve firms' optimality 
conditions. Their first order conditions may be simplified to

$$
\left(\delta_{i, t} z_{t}\right)^{1-\eta}=\mu_{i, t}^{-\eta} P_{i, t} \varepsilon_{i, t} \eta^{\eta} \quad \forall i, t
$$

which along with price normalization in equation 12 are sufficient to pin down the equilibrium spot market prices and output. Therefore, equations 12 and 15 combined result in a system of $n+1$ equations and $n+1$ unknowns for every period $t$ that fully characterizes the equilibrium solution of the model. The following result shows that, under the Cobb-Douglas case, i.e. $\nu=1$, this system of equation may be solve analytically.12

Theorem. When $\nu=1$, the equilibrium consumption expenditure growth is given by:

$$
\log \mathcal{C}_{t+1}-\log \mathcal{C}_{t}=\frac{1}{1-\eta}\left[\eta \Delta \mathcal{N}_{t+1}^{\mathcal{S}}-(1-\eta) \Delta \mathcal{N}_{t+1}^{\mathcal{C}}+\Delta e_{t+1}\right]
$$

where $\Delta \mathcal{N}_{t+1}^{\mathcal{S}}=\mathcal{N}_{t+1}^{\mathcal{S}}-\mathcal{N}_{t}^{\mathcal{S}}, \Delta \mathcal{N}_{t+1}^{\mathcal{C}}=\mathcal{N}_{t+1}^{\mathcal{C}}-\mathcal{N}_{t}^{\mathcal{C}}, \Delta e_{t+1}=e_{t+1}-e_{t}$, and

$$
\begin{aligned}
\mathcal{N}_{t}^{\mathcal{S}} & =\sum_{i} \delta_{i, t} \sum_{j} w_{i j, t} \log w_{i j, t} \\
\mathcal{N}_{t}^{\mathcal{C}} & =\sum_{i} \delta_{i, t} \log \delta_{i, t} \\
e_{t} & =\sum_{i} \delta_{i, t} \log \varepsilon_{i, t} .
\end{aligned}
$$

This is the main result of the general equilibrium model. Equation 16 shows that the consumption expenditure growth rate may be decomposed into three distinct factors: innovations in network sparsity $\left(\Delta \mathcal{N}_{t+1}^{\mathcal{S}}\right)$, network concentration $\left(\Delta \mathcal{N}_{t+1}^{\mathcal{C}}\right)$, and residual TFP $\left(\Delta e_{t+1}\right)$. According to equation 16 , changes in sparsity and residual TFP increase consumption and output growth, while changes in concentration have the opposite effect. In the next section, the relation between these factors and consumption growth is discussed in details.

\footnotetext{
${ }^{12}$ Atalay (2014) estimate the elasticity of substitution between inputs finding that the elasticity of substitution between inputs should be less than one (their point estimate is 0.034) when these inputs are not used to accumulate capital, however the elasticity of substitution between inputs used to investment and build capital should be greater than 1 (2.87). This means that firms' input are more substitutable when they are used to building capital than when they are used as raw materials. In my model, there is no capital accumulation, firms buy inputs from each other and these input are immediately transformed into effective investment or capital $\left(I_{i, t}\right)$ which is then used to produce the final output. Thus, neither of the two elasticities estimated by Atalay (2014) fully represent the elasticity parameter $\nu$, intuitively $\nu$ should be somewhat between both estimates. In the appendix D, I solve a first-order approximation of the model around $\nu=1$.
} 


\subsection{Network Factors}

Residual TFP Firms' productivity is combined into one aggregate variable given by

$$
e_{t} \equiv \sum_{i=1}^{n} \delta_{i, t} \log \varepsilon_{i, t}
$$

which is a weighted average of sector-specific productivities and the weights are given by firms' output share.

Since, the model doesn't have labor market nor capital accumulation, output growth is net of capital and labor utilization, which is exactly what econometricians estimate as TFP in the data. Therefore, the residual TFP, $e_{t}$, is TFP net of network factors. Innovations in the residual TFP, $\Delta e_{t+1}$, positively affects consumption growth, because firms become more productive on average.

Network Concentration The network concentration factor is given by

$$
\mathcal{N}_{t}^{\mathcal{C}} \equiv \sum_{i=1}^{n} \delta_{i, t} \log \delta_{i, t}
$$

This is the average of firms' log output share weighted by their own output share. This factor is exactly the negative entropy of the output share distribution and captures output share concentration. In equilibrium, sectoral shares depend primarily on the input-output network and the dynamics of concentration depends only on the input-output network dynamics. As discussed earlier, the output shares in equilibrium are equal to firms' centrality in the network; and, therefore, the network concentration factor measures the concentration of nodes' centrality which is equivalent to the concentration over the size of network nodes.

From equation 16, changes in concentration negatively affect consumption growth. An economy with high concentration has few large sectors with lower return to input investment due to decreasing returns to scale. These large sectors' lower productivity spreads across sectors through equilibrium prices, and, as a result, aggregate consumption and output decrease. Thus, high concentration leads to lower aggregate consumption.

Network Sparsity The network sparsity factor is given by

$$
\mathcal{N}_{t}^{\mathcal{S}} \equiv \sum_{i=1}^{n} \delta_{i, t} \underbrace{\sum_{j=1}^{n} w_{i j_{t}} \log w_{i j, t}}_{\equiv \mathcal{N}_{i, t}^{\mathcal{S}}} .
$$


Sparsity measures how thick and scarce network linkages are. Similar to the concentration factor, the term $\mathcal{N}_{i, t}^{\mathcal{S}}=\sum_{j=1}^{n} w_{i j_{t}} \log w_{i j, t}$ measures the concentration of $\left\{w_{i j, t}\right\}_{j}$, that is, the concentration of row $i$ of the network:

$$
W_{t} \equiv\left(\begin{array}{ccc}
w_{11, t} & \ldots & w_{1 n, t} \\
\vdots & \ddots & \vdots \\
w_{n 1, t} & \ldots & w_{n n, t}
\end{array}\right)
$$

Sparsity is the average, weighted by sectoral shares, of the concentration over input expenditures. High network sparsity factor implies that the input shares are concentrated and the network is sparse. Graphically, a network with low sparsity is represented by the network in panel (a) of Figure 2, while a network with high network sparsity are represented by the network in panels (b) and (c) of the same Figure.

When holding both the residual TFP and the concentration factors fixed, changes in sparsity positively affect consumption growth based on equation 16. The intuition behind these results is explained in three steps. First, I discuss the implications of changes in sparsity for firms' production function. Second, I discuss how the firms' optimality conditions change, and, third, I show that sparsity increases consumption growth when concentration is kept constant.

First, let's consider the effects of changes in sparsity on firms' production function. Firm $i$ 's output results from a combination of inputs acquired from other sectors, specifically its output is given by $Y_{i, t}=\varepsilon_{i, t} I_{i, t}^{\eta}$, where $I_{i, t}=\prod_{j=1}^{n} y_{i j, t}^{w_{i j, t}}$ is $i$ 's investment and $y_{i j, t}$ is the input from sector $j$ (see equations 1 and 2 when $\nu=1$ ). When sparsity increases, the shape of the investment function changes and the network weights become more concentrated, affecting the marginal product of each input. Hence, input allocations may become more or less productive, depending on specific changes in the network.

Figure 3 plots 2 different isoquants when there are only two inputs and sparsity increases. For firm 1, the weight on input 1 decreases from 0.5 to 0.1 , and its isoquant pivots clockwise (left panel). For firm 2, the weight on input 1 increases from 0.5 to 0.9 , and its isoquant pivots counter-clockwise (right panel). The isoquant pivots at the $45^{\circ}$ degree line and the firm becomes more productive if it uses more inputs from the sector whose weight was increased: firm 1 is more productive in the area above the $45^{\circ}$ degree line and firm 2 is more productive in the area below the line. To provide further intuition why this is the case, let's consider firm 1. When the weight on input 1 decreased from 0.5 to 0.1 , the weight on input 2 increased from 0.5 to 0.9 , and, as a result, firm 1 becomes better at coverting input 2 into final output. If firm 1 has more of input 2, then it's able to produce more after the increase 
Figure 3: Sparsity and Isoquants

This Figure plots output isoquants for 2 goods, i.e. $I_{i}=y_{1}^{w_{i, 1}} y_{2}^{w_{i, 2}}$, and 2 different sparsity levels: low sparsity (solid line) and high sparsity (dashed line). The Figure also plots a $45^{\circ}$ degree line as well as an isocost with slope of -1 .
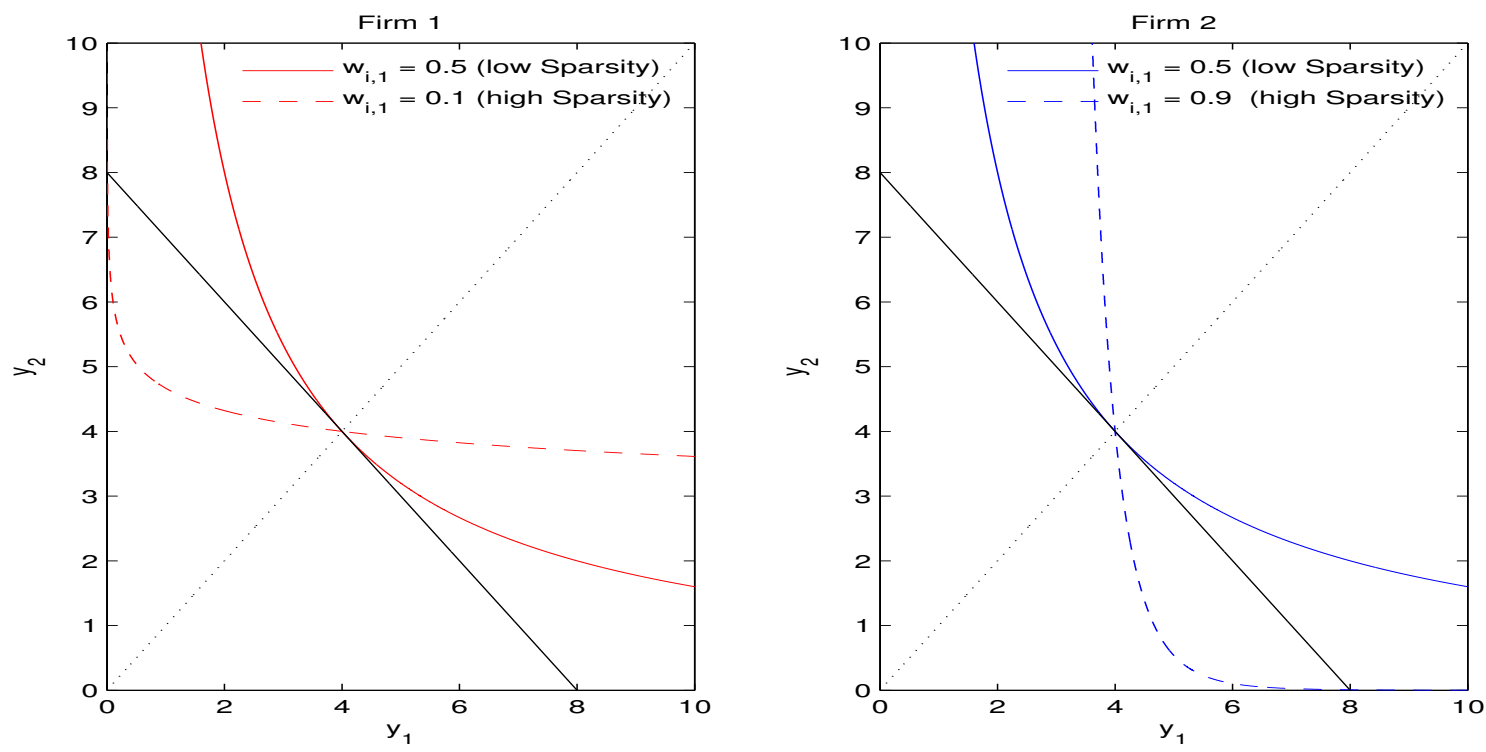

in sparsity, that is, all input combinations above the $45^{\circ}$ degree line result in a higher final output for firm 1. For a given input allocation of firm $i$, a higher weight $w_{i j, t}$ increases the marginal product of input $j$. Thus, more concentrated weights, $\left\{w_{i j, t}\right\}_{j}$, increase the final output if firm $i$ has more inputs from sectors whose weights were increased. Therefore, changes in sparsity affect the productivity of the firms depending on their input allocations.

The second step to understand why sparsity increases consumption is to understand how sparsity affects firms' optimality conditions. As a result of profit maximization, firms spend less on inputs from sectors whose network weights were decreased and more on inputs from sectors whose weights were increased. In the example, firm 1 spends less on input 1 and more on input 2, and firm 2 spends more on input 1 and less on input 2. Thus, an increase in firm $i$ sparsity makes firms' input allocation to head towards a more productive input allocation. If firm $i$ becomes more or less productive depends both on the change in sparsity and on the spot market prices, because firms substitute inputs at the relative spot market prices.

Solving firm $i$ 's first order condition for the total output yields

$$
P_{i, t} Y_{i, t}=\frac{\varepsilon_{i, t}^{\frac{1}{1-\eta}} \eta^{\frac{\eta}{1-\eta}} P_{i, t}^{\frac{1}{1-\eta}}}{\left(\prod P_{j, t}^{w_{i j, t}}\right)^{\frac{\eta}{1-\eta}}} \exp \left\{\frac{\eta}{1-\eta} \mathcal{N}_{i, t}^{\mathcal{S}}\right\}
$$

Therefore, changes in the network weights $\left\{w_{i j, t}\right\}$ towards more concentrated input shares 
has two immediate effects. On the one hand, it increases total output as it directly increases the last term in expression 17. The intuition behind this effect is that firm $i$ substitutes inputs towards a more productive input allocation. This is the same intuition of firm 1 using more good 2 as input and being above the $45^{\circ}$ degree line (Figure 3, left panel).

On the other hand, firms substitute inputs at their relative spot market prices, and changes in input combination affects the marginal cost of production. After sparsity increases, a particular firm may use inputs that are relatively more (less) expensive, causing marginal cost of production to increase (decrease) and final output to decrease (increase). Therefore, changes in marginal cost may have a positive or negative effect on output of the firms, depending both on the spot market prices and on the specific changes in the network. This is captured by changes in the denominator term $\prod P_{j, t}^{w_{i j, t}}$ in equation 17 . The aggregate effect on the output of the firms depends on which effect dominates.

The third step to understand why sparsity increases consumption is to consider the aggregate effect when we keep concentration constant. When concentration is kept constant, the cost effect is averaged out to zero and it has no aggregate effect in equilibrium. The intuition may be obtained through a partial equilibrium exercise in which sparsity factor increases, but sectors' shares and productivity are kept constant. Changes in the final output can be approximated by

$$
\log z_{t+1}-\log z_{t} \approx \sum_{i=1}^{n} \delta_{i, t}\left[\log \left(P_{i, t+1} Y_{i, t+1}\right)-\log \left(P_{i, t+1} Y_{i, t+1}\right)\right]
$$

Using the above approximation, we may substitute in the final output of each firm from equation 17. Moreover, keeping both prices and output shares constant, the total output growth may be approximated by

$$
\begin{aligned}
\log z_{t+1}-\log z_{t} & \approx \frac{\eta}{1-\eta}\left(\sum_{i=1}^{n} \delta_{i, t+1} \mathcal{N}_{i, t+1}^{\mathcal{S}}-\sum_{i=1}^{n} \delta_{i, t} \mathcal{N}_{i, t}^{\mathcal{S}}\right)+\frac{\eta}{1-\eta}\left(\sum_{i=1}^{n} \delta_{i, t} \sum_{j=1}^{n}\left(w_{i j, t+1}-w_{i j, t}\right) \log P_{j, t}\right) \\
& =\frac{\eta}{1-\eta}\left(\mathcal{N}_{t+1}^{\mathcal{S}}-\mathcal{N}_{t}^{\mathcal{S}}\right)+\frac{\eta}{1-\eta}(\sum_{j=1}^{n} \log P_{j, t} \underbrace{\sum_{i=1}^{n} \delta_{i, t}\left(w_{i j, t+1}-w_{i j, t}\right)}_{=0}) \\
& =\frac{\eta}{1-\eta}\left(\mathcal{N}_{t+1}^{\mathcal{S}}-\mathcal{N}_{t}^{\mathcal{S}}\right)
\end{aligned}
$$

In the second line, the term $\sum_{i=1}^{n} \delta_{i, t}\left(w_{i j, t+1}-w_{i j, t}\right)$ is zero based on the market clearing 
conditions and on the assumption that output shares are constant ${ }^{13}$ This means that the cost effect is averaged out to zero. Some firms use more inputs that are relatively more expensive, while others use more inputs that are relatively less expensive, but the aggregate effect is zero.

At the macro level, the efficiency effect dominates the cost effect and aggregate output increases as sparsity increases. Thus, when sparsity is high, the input-output linkages change causing aggregate consumption to increase, and changes in sparsity positively affects consumption growth as described by equation 16 .

\subsection{Examples}

Concentration and sparsity represents distinct attributes of a network. In this section, I provide an example of networks with the same concentration, but different sparsity level, and another example in which concentration varies while keeping sparsity constant. In addition, one may ask whether it is possible to recover the entire network based on concentration and sparsity alone. I show this is not possible, by discussing a third example of two distinct networks that have exactly the same network factors.

Example I: change in network sparsity. Let's consider an example of an economy with 2 sectors. To keep the example simple, let's assume that the household weights on each good is the same.

Let's consider two distinct networks. In the first one, all entries in the network are equal:

$$
W_{1}=\left(\begin{array}{cc}
0.5 & 0.5 \\
0.5 & 0.5
\end{array}\right)
$$

In this case, the network is completely symmetric and both sectors have the same output share in equilibrium: $\delta_{1}^{\text {Network } 1}=\delta_{2}^{\text {Network } 1}=0.50$. Furthermore, concentration and sparsity are both -0.69 . This is represented graphically in panel (a) of Figure 4.

In the second network, sector 1 spends $90 \%$ of its input investments on inputs from sector 2 and only $10 \%$ on inputs from sector 1 , and sector 2 does exactly the opposite:

$$
W_{2}=\left(\begin{array}{ll}
0.1 & 0.9 \\
0.9 & 0.1
\end{array}\right)
$$

Sector 1 isoquant pivots counter-clockwise while sector 2 isoquants pivots clockwise (see

\footnotetext{
${ }^{13}$ This is an immediate implication of the equilibrium output shares defined in equation 14
} 
Figure 4: Network Factors and Network Representation

This picture contains the representation of 3 different networks:

$$
W_{1}=\left(\begin{array}{cc}
0.5 & 0.5 \\
0.5 & 0.5
\end{array}\right), W_{2}=\left(\begin{array}{cc}
0.1 & 0.9 \\
0.9 & 0.1
\end{array}\right), \text { and } W_{3}=\left(\begin{array}{cc}
0.9 & 0.1 \\
0.9 & 0.1
\end{array}\right)
$$

The edge arrow represents the input flow and its width represents the network weight. Networks 1 and 2 have the same concentration factor, but different sparsity factor with network 2 being more sparse than network 1 . Networks 2 and 3 have the sparsity, but different concentration factors - network 3 is more concentrated.

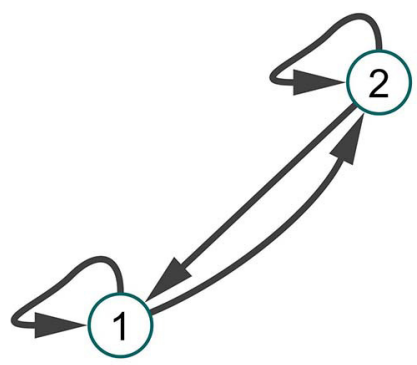

(a) Network 1

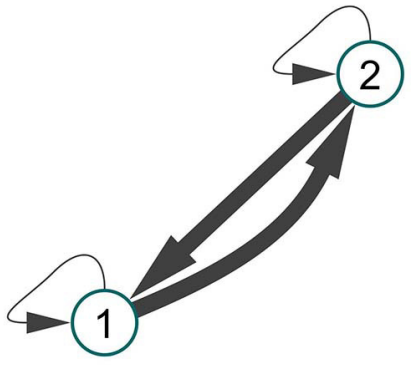

(b) Network 2

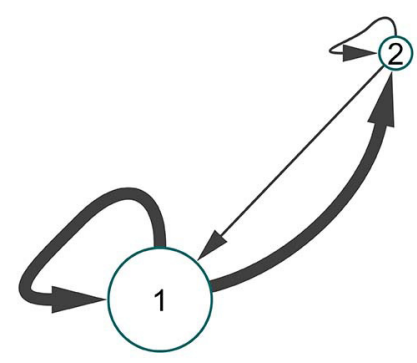

(c) Network 3

Figure 3). In equilibrium, sector 2 chooses input combination below the $45^{\circ}$ degree line and is to produce more output. Similarly, sector 1 input combination is above the $45^{\circ}$ degree line and is able to produce more output as well. Moreover, the network is symmetric and output shares are the same: $\delta_{1}^{\text {Network } 2}=\delta_{2}^{\text {Network } 2}=0.50$, which results in a concentration factor of -0.69 , same as in the first network. However, there is more input concentration and network sparsity is -0.33 . This means that on average firms are more productive through an increase in sparsity without affecting concentration.

Example II: change in network concentration. Keeping the structure of example 1, let's consider a third network similar to network 2 , but firm 1 will have the same network weights as firm 2:

$$
W_{3}=\left(\begin{array}{ll}
0.9 & 0.1 \\
0.9 & 0.1
\end{array}\right) .
$$

This network is represented by panel (c) in Figure 4. In this case, both sectors are using less inputs from sector 2 and more inputs from sector 1. Sectors 1 and 2 spend 90\% of their input investment in goods from sector 1 and only $10 \%$ in goods from sector 2 . As a result, sector 1 is larger than sector $2: \delta_{1}^{\text {Network } 3}=0.66$ and $\delta_{2}^{\text {Network } 3}=0.34$. In the Figure, sectoral shares are represented by nodes' size. Network concentration is -0.64 , which is greater than the concentration of both first and second networks, but sparsity factor is the same of the second network at -0.33 . Both sectors rely more on sector 1 , and, in equilibrium, sector 1 
Figure 5: Different Networks with same Factors

This Figure plots two distinct network that have the same network factors. The two networks are given by

$$
W_{4}=\left(\begin{array}{ccc}
0.4 & 0.4 & 0.2 \\
0.4 & 0.4 & 0.2 \\
0.4 & 0.4 & 0.2
\end{array}\right), \text { and } W_{5}=\left(\begin{array}{ccc}
0.48 & 0.26 & 0.26 \\
0.48 & 0.26 & 0.26 \\
0.48 & 0.26 & 0.26
\end{array}\right)
$$

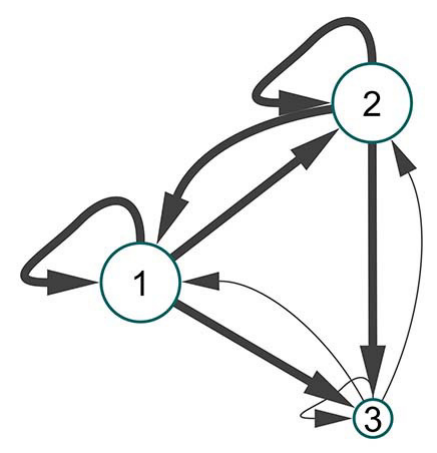

(a) Network 4

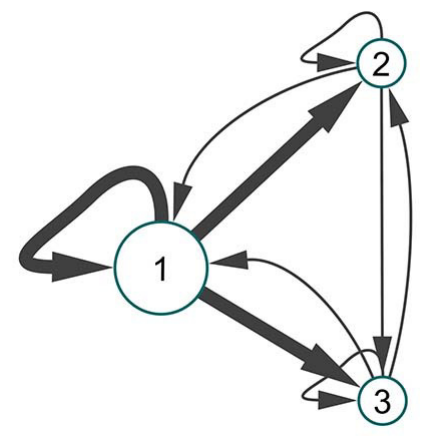

(b) Network 5

is twice as large as sector 2 . As a result, sector 1 has lower return to capital (investment) decreasing aggregate consumption in equilibrium.

Example III: same network factors, but different networks. Finally, given the two network factors, it's not possible to recover the entire network. Figure 5 shows two distinct networks that have the same network factors. In the fourth network, sectors 1 and 2 are the largest ones with output share of 0.4 each, the network concentration factor is -1.09, and network sparsity factor is -1.05 . In the fifth network, sector 1 is the largest sector with 0.48 output share and sectors 2 and 3 split the remaining output share, 0.26 each. Although network 5 is different from network 4 , they have the same network factors.

\section{Evidence from the data}

The multisector network model predicts that consumption growth depends positively on sparsity and negatively on concentration (equation 16) 14 A positive shock to sparsity is associated with higher consumption and lower marginal utility, while a positive shock to

\footnotetext{
${ }^{14}$ Changes in the network factors are correlated with consumption growth of the share holder in a lower frequency. Using shareholder consumption data from Annette Vissing-Jørgensen's website and regressing consumption growth on my network factors, I show that sparsity is associated with higher consumption growth and concentration with lower growth rates, over the next three to five years. (Malloy, Moskowitz, and Vissing-Jørgensen 2009)
} 
concentration is associated with lower consumption and higher marginal utility. Therefore, the model has a clear-cut prediction regarding how innovations in the network factors should be priced: innovations in sparsity carry a positive price of risk and innovations in concentration carry a negative price of risk. In this section, I verify this prediction of the model by building sorted portfolios based on stocks' exposure to the innovations in the network factors. In addition, I construct factor-mimicking portfolio to verify, by estimating prices of risk, that concentration and sparsity are also priced in other portfolios such as industry, book to market, 25 Fama French, earnings price and volatility sorted portfolios.

\section{$3.1 \quad$ Data}

Network data The concentration factor may be computed directly from sectors' output shares, but sparsity requires the entire input-output network matrix. The main input-output data sources are the BEA Input-Output tables, however this data set is available on an annual basis only from 1997 to 2012. Due to the short BEA sample, I use an alternative data set to compute an estimate of the Input-Output table using the compustat segment customer data which is available from 1979 to 2013. If a customer represents more than $10 \%$ of the sellers' revenue, then the customer's name is reported in the compustat customer segment data as well as the sales amount to that particular customer 15 Combing this information with the total sales available in compustat, it's possible to reconstruct the network for each year and computed a time series for the network factors ${ }^{16}$ Firms are aggregated at the sector level (2-digit naics), and the detailed construction of the data is provided in Appendix F.

The two network factors are plotted in Figure 6. The correlation between the two in level is $-34 \%$ ( $\mathrm{p}$-value of 0.04 ). The factors' innovations are computed as the difference from one year to another, and the correlation between the innovations is $6 \%$ (p-value of 0.72). This suggests that factors' innovations are not correlated with each other, which means that innovations in sparsity and concentration represent two distinct sources of risk. Table 1 reports mean, standard deviation, and autocorrelation of the network factor, both in level and innovations. The factors are autocorrelated in levels, but not in the innovations.

Return data I consider all CRSP stocks with share codes 10, 11 and 12. Penny stocks are removed from the sample and delisting returns are taken into account. I also restrict the sample to stocks with network data in order to keep the network data consistent with

\footnotetext{
${ }^{15}$ Regulation SFAS No. 14 and SFAS No. 131.

${ }^{16}$ Cohen and Frazzini (2008) located the CRSP permanent number, PERMNO, of the customer until the year of 2009. I updated their data set by locating the customer identification number up to 2013.
} 
Figure 6: Network Factors

This Figure plots the time series of concentration (solid line) and sparsity (dashed line) computed from Compustat. The first panel plots both factors in level, and the second one plots the innovations (one period difference).
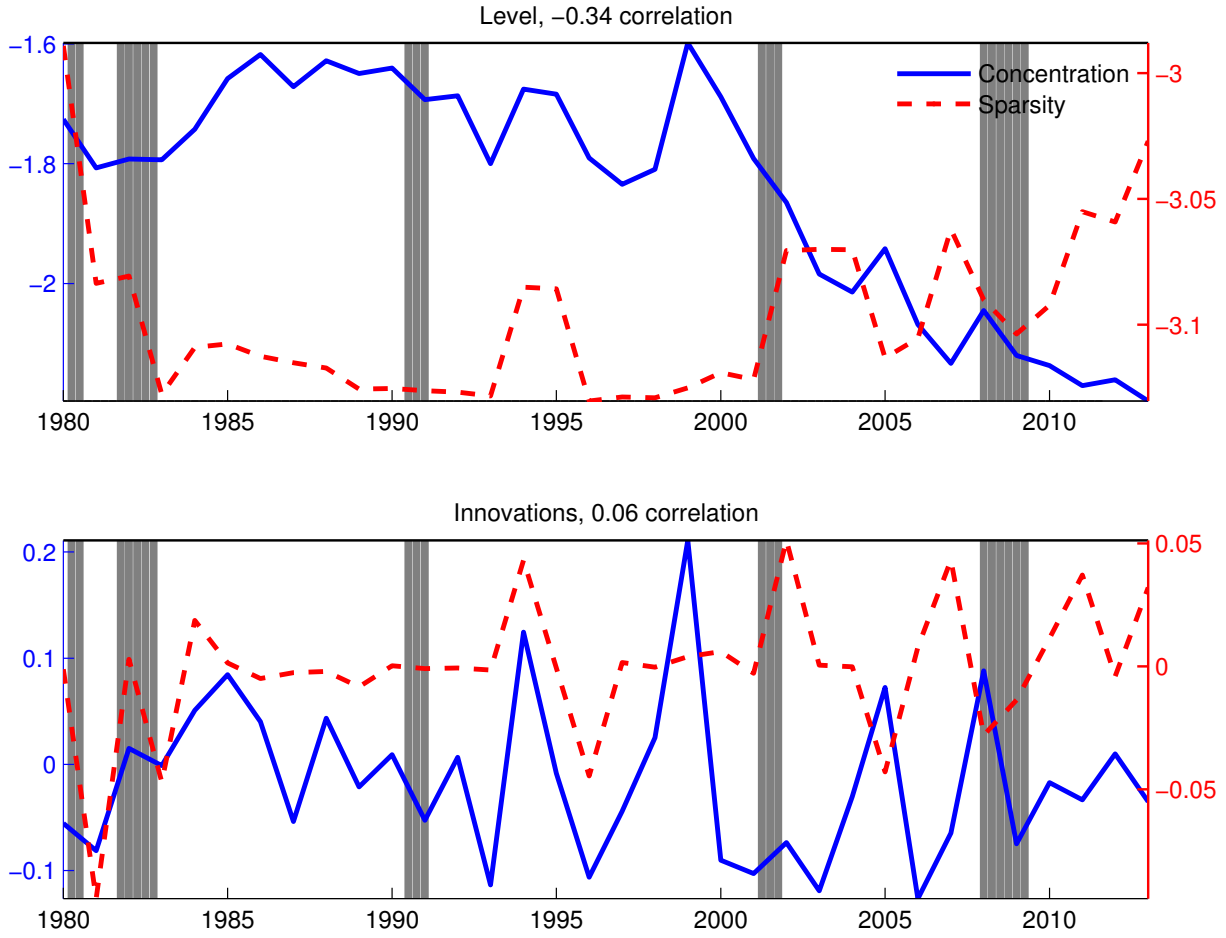

the return data. The annual risk-free rate and the annual market return are both from Ken French's website.

\subsection{Sorted Portfolios and Trading Strategy}

In order to verify the positive price of risk for sparsity innovations and negative price of risk for concentration innovations, I sort stocks based on their exposure to these innovations and form portfolios by terciles in a trailing window.

For every stock $i$, we can regress its excess return on a constant and on the innovation in the network factors controlling for changes in the residual TFP as well as network factors in level from the previous period: 17

$$
r_{t}^{i}=\alpha^{i}+\beta_{\mathcal{N}^{\mathcal{S}}}^{i} \Delta \mathcal{N}_{t}^{\mathcal{S}}+\beta_{\mathcal{N}^{\mathcal{c}}}^{i} \Delta \mathcal{N}_{t}^{\mathcal{C}}+\text { Controls }+\xi_{t}^{i},
$$

17 Changes in the residual TFP factor, i.e. $\Delta e_{t+1}$, are computed as the residual of regressing TFP growth on factor's innovations The TFP used is from the San Francisco FED (Fernald and Matoba 2009, Fernald 2012, Basu, Fernald, and Kimball 2006). The controls used in the benchmark estimation are not crucial for the sorted portfolio results as discussed in the robustness section 3.3 
Table 1: Network Factors Statistics

This Table reports statistics for the network factor in level and changes (Innov.). $\mathrm{AC}(j)$ stands for the $j^{\text {th }}$ auto correlation.

\begin{tabular}{lrr|rr} 
& \multicolumn{2}{c}{ Sparsity } & \multicolumn{2}{c}{ concentration } \\
& Level & Innov. & Level & Innov. \\
\hline Mean & -3.10 & -0.00 & -1.83 & -0.02 \\
Standard Deviation & 0.0360 & 0.0277 & 0.1817 & 0.0757 \\
\hline $\mathrm{AC}(1)$ & 0.67 & -0.09 & 0.91 & -0.07 \\
$\mathrm{AC}(2)$ & 0.25 & 0.02 & 0.82 & -0.20 \\
$\mathrm{AC}(3)$ & 0.09 & -0.12 & 0.77 & -0.06 \\
$\mathrm{AC}(4)$ & 0.04 & 0.14 & 0.73 & -0.01 \\
$\mathrm{AC}(5)$ & 0.08 & 0.08 & 0.70 & 0.06 \\
\hline
\end{tabular}

where coefficients $\beta_{\mathcal{N}^{\mathcal{S}}}^{i}$ and $\beta_{\mathcal{N}^{\mathcal{C}}}^{i}$ measure the exposure of stock $i$ to factors' innovations.

If sparsity carries a positive price of risk, then stocks with high sparsity-beta, i.e. high $\beta_{\mathcal{N}_{\mathcal{S}}}^{i}$, are risky assets and should have higher expected returns. Similarly, high concentrationbeta stocks should have lower expected return. For every year $t$, I compute stocks' exposure to innovations in the network factors from regression 18 over a 15-year year window from $t-14$ to $t$. Then, stocks are sorted based on each beta separately (one-way sort). Given that stocks are properly sorted, valued-weighted portfolios are formed over the subsequent year $(t+1)$. This procedure repeats as a trailing window until the last year of the sample.

The average returns of the one-way sorted portfolios are reported in Table 2 as well as return volatilities, post-sample CAPM and Fama-French alphas and other portfolio characteristics. Panel A reports these moments when portfolios are sorted based on stocks' exposure to network sparsity innovations. The high sparsity-beta portfolio earned $6.01 \%$ higher return than the low sparsity-beta portfolio. which is consistent with a positive price of risk for sparsity innovations. Panel B reports portfolios sorted by their exposure to concentration innovations, the return spread is $-4.04 \%$ and statistically significant. Moreover, the spread sign is consistent with a negative price of price as predicted by the model. Interestingly, neither return gaps can be explained by the CAPM or the Fama French three-factor model. The second and third rows of each panel report the post-sample alphas and the alpha spread is statistically significant. This suggests that innovations in the network factors represent a source of risk that is not captured by the market return (CAPM) or by the Fama French factors. For each trailing window, we can compute the correlation between the sparsity and concentration betas estimated from regression 18 . The average correlation is $-14 \%$, which means that betas are not very correlated and the two factors represent distinct sources of risk. 
Table 2: One Way Sorted Portfolios

\begin{tabular}{lccccc}
\hline \multicolumn{5}{c}{ Panel A: Sparsity } \\
& $(1)$ & $(2)$ & $(3)$ & $(3)-(1)$ & t-stat \\
Avg. Exc. Returns (\%) & 5.24 & 8.61 & 11.25 & 6.01 & 2.26 \\
$\alpha_{C A P M}$ & -3.15 & 2.29 & 4.78 & 7.92 & 3.11 \\
$\alpha_{F F}$ & -3.21 & 1.47 & 3.84 & 7.04 & 2.91 \\
Volatility (\%) & 17.60 & 13.78 & 15.13 & 11.60 & - \\
Book/Market & 0.76 & 0.67 & 0.70 & - & - \\
Avg. Market Value (\$bn) & 1.53 & 2.18 & 1.23 & & \\
\hline \multicolumn{5}{c}{ Panel } & B: Concentration \\
Avg. Exc. Returns (\%) & $(1)$ & $(2)$ & $(3)$ & $(3)-(1)$ & t-stat \\
$\alpha_{C A P M}$ & 10.23 & 8.51 & 6.19 & -4.04 & -2.19 \\
$\alpha_{F F}$ & 2.62 & 2.43 & -1.60 & -4.21 & -2.26 \\
Volatility (\%) & 2.00 & 1.64 & -2.00 & -4.01 & -2.12 \\
Book/Market & 16.18 & 13.60 & 16.27 & 8.05 & - \\
Avg. Market Value (\$bn) & 0.74 & 0.69 & 0.70 & - & - \\
\hline \hline
\end{tabular}

The sorted portfolios are roughly similar to each other. The first tercile for both sparsitybeta and concentration-beta sorted portfolios have slightly higher book to market ratio (0.76 and 0.74 ) than the other two terciles (roughly 0.7 each). The average market value of stocks is reported in the last row of each panel.

Altogether, there is compelling evidence that innovations in concentration and sparsity factors constitute priced sources of risk. Moreover, the two factors represent distinct sources of risk that cannot be explained by standard asset pricing model such the CAPM or the Fama French three-factor model.

Market betas For the market portfolio, we can compute the factor betas from regression 18 using the market return instead of individual stock returns. The market sparsity beta is estimated at 0.40 and the market concentration beta at -0.08 . The betas are not significant, but their signs are consistent with the model. If aggregate consumption growth depends positively on sparsity innovations, then the market sparsity-beta should be positive. Similarly, the market concentration-beta should be negative.

\subsection{Robustness}

My results are robust to different specifications of my estimation procedure. I build doublesorted portfolios based on both network factors, remove network factors in level from the 
control variables, change the CRSP sample considered, change the residual TFP calculations, and change the trailing window length up to 20 years. In addition, I double sort stocks on my network factors and on other risk factors and stock characteristics documents by the asset pricing literature, including market value, book to market ratio, total and idiosyncratic volatility, volume and turnover. Tables 6, 7, 8, 9, and 10 have robustness estimations and are reported in appendix $\mathrm{F}$

Double sort In this robustness exercise, I sort stocks on both factors simultaneously and build double-sorted portfolios. Table 6 reports returns, and CAPM as well as Fama French alphas for the double sorted portfolios. The return spread on sparsity-beta sorted portfolios is $11.26 \%$ per year among stocks with high concentration betas, however for lower concentration betas the return spread is lower and not significant. The return on the concentration-beta sorted portfolio loses significance for all sparsity betas terciles. Thus, the sparsity factor seems to survive double sorting.

Exposure estimation I consider an alternative set of controls in regression 18 by removing the network factors in level. In this case, the control variables only include the changes in the residual TFP factor. According to the model, this shouldn't affect the estimation of the exposure coefficients (betas). Row 2 in panel (a) of Table 7 reports the one way sorted portfolio returns. Panel (b) and (c) report the CAPM and Fama French post sample alphas. The return spread decreases for both sparsity and concentration. The return spread as well as the CAPM and Fama-French alphas are significant for the sparsity-beta sorted portfolios. However, the return spread of the concentration-beta sorted portfolios loses significance, even though the CAPM alpha spread is significant.

CRSP sample In the benchmark estimation, I considered only CRSP stocks with network data available. Considering all CRSP stocks, the one-way sorted portfolio results still hold and return spread are statistically significant and of the same magnitude (row 3 in Table 7) 18

As an out of sample test, I consider only stocks without network data. Interestingly, in the out of sample test (row 4 in Table 7), the sparsity-beta sorted portfolio have a return spread of nearly zero and not significant, while the return spread of the concentration-beta sorted portfolio is significant at $10 \%$. Thus, innovations in the concentration factor seem to be a priced source of risk even among stocks without network data.

\footnotetext{
${ }^{18}$ The sample is still restricted to stocks with share codes of 10,11 or 12 , no penny stocks and at least 16 years of data in order to compute the network factors betas.
} 
Residual TFP In the benchmark estimation, changes in the residual TFP factor are computed as the residual of regressing TFP growth on changes in the network factors. I consider four alternative specifications as well. I use aggregate consumption growth rather than TFP growth (row 5), exclude residual TFP factor from controls in regression 18 when estimating network betas (row 6), use equation 16 to directly compute the residual TFP, but using aggregate consumption growth rather than TFP growth (row 7), and use equation 16 to directly compute the residual TFP using reasonable returns to scale $\eta$ (row 8).19

The results on the sparsity-beta sorted portfolios remain roughly unchanged. For the concentration-beta sorted portfolios, the results are unchanged only for (i) and (ii). However, (iii) and (iv) induce significant correlation between the estimated betas. Table 8 reports the time series average of the cross-section correlation between the estimated betas. In the benchmark estimation, the average correlation between the sparsity and concentration betas is -0.14 (row 1 ), but it is roughly -0.80 when the residual TFP factor is computed directly from equation 16 (rows 7 and 8).

Trailing window In the benchmark estimation, the trailing window is 15 years. The quantitative results are robust to using trailing windows of 16 years and up to 20 years (rows 9 to 13 in Table 7 ).

Double sort on other factors Table 9 reports portfolios double sorted on sparsity and 7 other factors: market value, book to market ratio, total volatility (over 1 year of daily data), idiosyncratic volatility from CAPM model (over 1 year of daily data), idiosyncratic volatility from Fama French three-factor model (over 1 year of daily data), volume and turnover (volume divided by market value). Table 10 reports portfolios double sorted on concentration and on the same 7 other factors. Both the sparsity and concentration factors are strong among stocks with high market value, low book to market (growth firms), low volatility, high volume and low turnover.

\subsection{Fama MacBeth Regressions}

In the previous section, I show that innovations in concentration and sparsity are priced such that high sparsity-beta stock earn higher average returns than stock with low sparsitybeta, and high concentration-beta stock earn lower average returns than stock with low concentration-beta. In addition, we may verify whether the network factors are priced in

\footnotetext{
${ }^{19} \eta$ represent the returns to scale to capital, thus I use $\eta=0.35$. The quantitative results don't change much for $\eta$ between 0.2 and 0.5 .
} 
Table 3: Fama-MacBeth Regressions

This Table reports the prices of risk estimated from Fama MacBeth regression and t-stat are adjusted for heteroscedasticity. I consider two sets of asset pricing factors: (1) market excess return which is the CAPM model, and (2) the factor-mimicking portfolios along with the market excess returns. In terms of test assets, I consider 8 different test assets: (a) 10 sparsitybeta sorted portfolios, (b) 10 concentration-beta sorted portfolios, (c) 10 sparsity-beta sorted and 10 concentration-beta sorted portfolios, (d) 30 industry portfolios (French's website), (e) 10 book to market portfolios (French's website), (f) 10 idiosyncratic volatility sorted portfolios build from monthly data using residual from CAPM model (Ang, Hodrick, Xing, and Zhang 2006), and $(\mathrm{g})$ all the previous portfolio combined, and $(\mathrm{h})$ same as previous column but adding 10 market equity and 10 momentum sorted portfolios. The sample is from January 1995 to December 2013 on monthly frequency, and all coefficients and root mean square errors (RMSE) are annualized.

(a) 10 Sparsity (b) 10 Concentration (c) 10 Sparsity and 10 Concentration
Constant

t-stat

Mkt-rf

t-stat

Sparsity

t-stat

Concentration

t-stat

$R^{2}$

RMSE

\section{(1)}

19.27

7.73

$-12.24$

$-4.95$

$-$

-
$-\quad 9.61$

$\begin{array}{ll}-\quad & -8.45\end{array}$

$\begin{array}{ll}- & -4.79\end{array}$

0.55

1.71
(2)

7.21

1.86

0.95

0.23

4.58

$-4.79$

1.81

\section{(1)}

4.48

3.07

4.46

3.84

$-$

$-\quad-2.25$

$\begin{array}{ll}- & -0.89\end{array}$

$\begin{array}{ll}- & -2.26\end{array}$

$\begin{array}{ll}- & -2.21 \\ 0.12 & 0.29\end{array}$

0.12

1.95
(2)

6.66

1.88

2.29

0.65

0.29
(1) (2)

$11.31 \quad 3.15$

$\begin{array}{ll}3.36 & 0.77\end{array}$

$\begin{array}{ll}-3.16 & 5.19\end{array}$

$-0.81 \quad 1.16$

$\begin{array}{ll}- & 3.50\end{array}$

$-\quad 3.32$
$-\quad 3.50$

$\begin{array}{ll}- & -2.09 \\ -\end{array}$

$\begin{array}{ll}- & -1.26\end{array}$

0.05

2.28 (e)

10 Book to Market

(1)

Constant

t-stat

Mkt-rf

t-stat

Sparsity

t-stat

Concentration

t-stat

$R^{2}$

RMSE
(2)
2.58
0.65
8.80
2.24
7.13
3.32
$-5.29$
0.53
0.63

9.13

1.84

3.15

0.57

$-5.02$ (f)

10 Idiosyncratic

Volatility (g)

All

0.37

1.85 (d)

30 Industry

(1) (2)

$\begin{array}{ll}11.89 & 10.46\end{array}$

$8.80 \quad 7.28$

$\begin{array}{ll}0.40 & 0.70\end{array}$

$\begin{array}{ll}0.27 & 0.52\end{array}$

0.50
$-\quad 4.50$

$-\quad 2.18$
$-\quad-7.07$

$\begin{array}{ll}- & -7.07\end{array}$

$\begin{array}{ll}- & -2.64\end{array}$

$\begin{array}{ll}0.00 & 0.32\end{array}$

$\begin{array}{ll}3.18 & 2.63\end{array}$

(h)

All + 10 Size and 10 momentum

(1) (2)

$13.29 \quad 10.37$

$\begin{array}{ll}11.21 & 6.42\end{array}$

$\begin{array}{lllll}4.06 & -2.42 & 1.25 & -2.37 & 0.01 \\ 2.19 & -1.99 & 0.89 & -1.96 & 0.01\end{array}$

\begin{tabular}{l|cc|cc}
2.19 & -1.99 & 0.89 & -1.96 & 0.01 \\
9.34 & - & 5.65 & - & 4.44
\end{tabular}

$\begin{array}{llllll}3.83 & - & 3.64 & - & 2.92\end{array}$

$\begin{array}{llllll}-3.92 & - & -3.87 & - & -5.47\end{array}$

$\begin{array}{lllllll}-1.38 & - & -1.91 & - & -2.71\end{array}$

\begin{tabular}{l|llll}
0.74 & 0.06 & 0.34 & 0.05 & 0.28
\end{tabular}

\begin{tabular}{l|lllll}
0.95 & 3.17 & 2.66 & 3.05 & 2.66
\end{tabular}

other portfolios as well. We may build factor-mimicking portfolios by going long on the high beta portfolio (3rd tercile) and short on the low beta portfolio (1st tercile), then we may use the factor-mimicking portfolios and run Fama MacBeth regressions. We can do this exercise for any set of portfolios verifying whether innovations in sparsity and concentration are priced.

The Fama MacBeth regression is a two-stage procedure. For a given set of test assets and a given set of asset pricing factors, we first regress the time-series excess returns on the factors considered, one regression for each asset. Then, in the second stage, we regress the 
time-series average of the excess returns on the estimated coefficients from the first stage. The coefficients of the second stage are the prices of risk of the asset pricing factors.

I consider two sets of asset pricing factors: (1) market excess return which is the CAPM model, and (2) the factor-mimicking portfolios along with the market excess returns. In terms of test assets, I consider 8 different test assets: (a) 10 sparsity-beta sorted portfolios, (b) 10 concentration-beta sorted portfolios, (c) 10 sparsity-beta sorted and 10 concentration-beta sorted portfolios, (d) 30 industry portfolios (French's website), (e) 10 book to market portfolios (French's website), (f) 10 idiosyncratic volatility sorted portfolios build from monthly data using residual from CAPM model (Ang, Hodrick, Xing, and Zhang 2006), and (g) all the previous portfolio combined, and (h) same as previous one but adding 10 market equity and 10 momentum sorted portfolios. The results are displayed in Table 3.

Innovations in either sparsity or concentration are priced in all test asset assets considered. For example, both factor-mimicking portfolios are priced in the 30 industry portfolio (panel d). The 30 industry portfolios are considered, because it's close to the number of sectors used in the construction of the network factors. Both factors are priced in the 10 portfolios sorted on book-to-market (panel e). In the 10 portfolios sorted by idiosyncratic volatility (panel g), only sparsity is priced. I also consider a larger set of portfolios including the sorted portfolio considered so far and in this large set of portfolios, both sparsity and concentration are priced (panel f). Sparsity and concentration are not priced in portfolios sorted by size and momentum, but when we add them to the set of test assets considered both sparsity and concentration are priced as predicted by my model.

\section{Calibration}

In this section, I verify whether the multisector network model is quantitatively consistent with the empirical evidence on return spreads. The model is calibrated to replicate the sorted portfolios from Table 2 as well as other asset pricing moments. First, I add more structure to model in order to solve for the stochastic discount factor and then I discuss the calibration process precisely.

\subsection{Setup}

The general equilibrium model specifies how innovations in network factors affect aggregate consumption (equation 16). However, the consumption claim in the model does not have leverage, and, in order to calibrate the model, both the levered and unlevered consumption 
processes have to be specified. First, let's specify the unlevered consumption claim by

$$
\begin{aligned}
\log z_{t+1}-\log z_{t} & =\phi_{\mathcal{N}^{c}} \Delta \mathcal{N}_{t+1}^{\mathcal{C}}+\phi_{\mathcal{N}^{\mathcal{s}}} \Delta \mathcal{N}_{t+1}^{\mathcal{S}}+\phi_{e} \Delta e_{t+1}+x_{t} \\
\Delta \mathcal{N}_{t+1}^{\mathcal{C}} & =\sigma_{\mathcal{N}^{c}} \varepsilon_{\mathcal{N}^{c}, t+1} \\
\Delta \mathcal{N}_{t+1}^{\mathcal{S}} & =\sigma_{\mathcal{N}^{\mathcal{S}} \varepsilon_{\mathcal{N}^{\mathcal{S}}, t+1}} \\
\Delta e_{t+1} & =\sigma_{e} \varepsilon_{e, t+1} \\
x_{t+1} & =\rho_{x} x_{t}+\sigma_{x} \varepsilon_{x, t+1}
\end{aligned}
$$

where $\varepsilon_{e, t+1}, \varepsilon_{\mathcal{N}^{c}, t+1}, \varepsilon_{\mathcal{N}^{s}, t+1}$ and $\varepsilon_{x, t+1}$ are i.i.d. standard normal random variables. This specification assumes that innovation in both network factors are i.i.d. shocks, which is consistent with the data. The expression for unlevered consumption has an extra term $x_{t}$ that is the long run risk factor based on Bansal and Yaron (2004). The long-run risk term is interpreted as a persistent component of expected consumption growth and is included to the unlevered consumption claim to generate equity risk premium consistent with the data. The long-run risk factor $x_{t}$ is quantitatively irrelevant to generate the return spread in the sorted portfolios. It's included to the model only to generate reasonable asset pricing moments for the market portfolio. In the model, $\log z_{t+1}-\log z_{t}$ is the growth rate of the unlevered consumption claim and loadings are given by

$$
\phi_{\mathcal{N C}}=-\frac{1}{1-\eta}, \quad \phi_{\mathcal{N} s}=\frac{\eta}{1-\eta} \quad \text { and } \quad \phi_{e}=\frac{1}{1-\eta},
$$

where $\eta$ is the return to scale.

Return on total wealth The first order approximation of the return on total wealth is given by

$$
r_{t+1}^{W}=\kappa_{0}^{c}+\Delta \log z_{t+1}+w c_{t+1}-\kappa_{1}^{c} w c_{t}
$$

where $\kappa_{0}^{c}=\log \left(\exp \left(\mu_{w c}\right)-1\right)+\frac{\exp \left(\mu_{w c}\right)}{\exp \left(\mu_{w c}\right)-1} \mu_{w c}, \kappa_{1}^{c}=\frac{\exp \left(\mu_{w c}\right)}{\exp \left(\mu_{w c}\right)-1}>1$ and $\mu_{w c}$ is the unconditional average of the wealth consumption expenditure ratio. One can guess that the wealth-consumption expenditure ratio is linear on the long run risk term:

$$
w c_{t}=\mu_{w c}+A x_{t}
$$

where $A=(1-\rho) \frac{\phi_{e} \phi_{x}}{\kappa_{1}^{c}-\rho_{x}}$ is a constant pinned down from the representative household Euler equation, and the approximation of the return on total wealth becomes 


$$
r_{t+1}^{W}=r_{0}^{c}+\beta_{x} x_{t}+\beta_{\varepsilon e} \sigma_{e} \varepsilon_{e, t+1}+\beta_{\varepsilon x} \sigma_{x} \varepsilon_{x, t+1}+\beta_{\varepsilon \mathcal{N}^{c}} \sigma_{\mathcal{N}^{c}} \varepsilon_{\mathcal{N}^{c}, t+1}+\beta_{\varepsilon \mathcal{N}^{\mathcal{S}}} \sigma_{\mathcal{N}^{\mathcal{S}}} \varepsilon_{\mathcal{N}^{\mathcal{S}}, t+1}
$$

where $r_{0}^{c}=\kappa_{0}^{c}-\mu_{w c}\left(\kappa_{1}^{c}-1\right), \beta_{x}=\rho, \beta_{\varepsilon e}=\phi_{e}, \beta_{\varepsilon x}=A, \beta_{\varepsilon \mathcal{N}^{c}}=\phi_{\mathcal{N}^{c}}$ and $\beta_{\varepsilon \mathcal{N}^{\mathcal{S}}}=\phi_{\mathcal{N}^{\mathcal{S}}}$. This is the return to unlevered consumption claim, not the market dividend itself. All detailed derivations and expressions are in Appendix E.

Stochastic discount factor The SDF is derived based on unlevered consumption claim and it's given by

$$
m_{t+1}=\mu_{s}+\lambda_{x} x_{t}-\lambda_{\varepsilon e} \sigma_{e} \varepsilon_{e, t+1}-\lambda_{\varepsilon x} \sigma_{x} \varepsilon_{x, t+1}-\lambda_{\varepsilon \mathcal{N}^{c}} \sigma_{\mathcal{N}^{c}} \varepsilon_{\mathcal{N}^{c}, t+1}-\lambda_{\varepsilon \mathcal{N}^{\mathcal{S}}} \sigma_{\mathcal{N}^{\mathcal{S}}} \varepsilon_{\mathcal{N}^{\mathcal{S}}, t+1}
$$

where

$$
\begin{aligned}
\mu_{s} & =\theta \log \beta+(\theta-1)\left(\kappa_{0}^{c}-\mu_{w c}\left(\kappa_{1}^{c}-1\right)\right) \\
\lambda_{x} & =-\gamma \phi_{e} \phi_{x}-(\theta-1) A\left(\kappa_{1}^{c}-\rho_{x}\right)=-\rho \phi_{e} \phi_{x} \\
\lambda_{\varepsilon e} & =\gamma \phi_{e} \\
\lambda_{\varepsilon x} & =-(\theta-1) A=(\gamma-\rho) \frac{\phi_{e} \phi_{x}}{\kappa_{1}^{c}-\rho_{x}} \\
\lambda_{\varepsilon \mathcal{N}^{\mathcal{C}}} & =\gamma \phi_{\mathcal{N}^{c}} \\
\lambda_{\varepsilon \mathcal{N}^{\mathcal{S}}} & =\gamma \phi_{\mathcal{N}^{\mathcal{S}}}
\end{aligned}
$$

The network factors' price of risk depends directly on $\phi_{\mathcal{N}^{c}}<0$ and $\phi_{\mathcal{N}^{\mathcal{S}}}>0$. Thus, innovations in network sparsity carries a positive price of risk while innovations in network concentration carries a negative price of risk.

Market Dividend The levered consumption claim (i.e. market dividend), however, may have loadings different from the unlevered claim. In a more general expression, let the growth rate of the levered consumption claim be given by

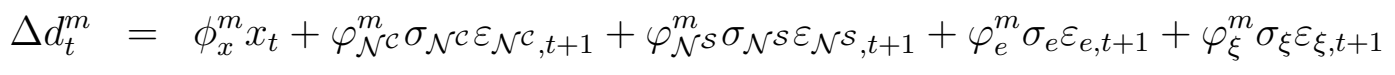

where the coefficients $\phi_{x}^{m}, \varphi_{V}^{m}, \varphi_{E}^{m}, \varphi_{e}^{m}$ and $\varphi_{\xi}^{m}$ are calibrated to match market return properties such as market betas, equity risk premium and market volatility. The process of leveraging consumption claim affects the exposure to the asset pricing factors: levered consumption may have betas different from the unlevered claim and the coefficients of the market 
dividend are post-leverage coefficients.

Portfolio Dividends Let the portfolio $i$ dividend growth process be described by

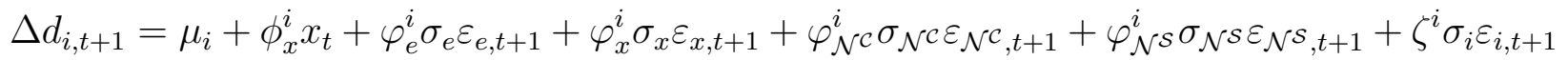

which is the projection of stock $i$ dividend onto the asset pricing factors. Similarly to the market portfolio, the coefficients of the dividends growth process are calibrated to match asset pricing moments of the portfolios.

Market and Portfolio Returns To compute the return to the market portfolio, or any other portfolio, we guess and verify that the $\log$ price-dividend ratio for portfolio $i$ is linear on the long-run risk term:

$$
p d_{i, t}=\mu_{i, p d}+A_{i} x_{t}
$$

where $A_{i}=\frac{\phi_{x}^{i}+\lambda_{x}}{1-\kappa_{1}^{i} \rho_{x}}$ is a constant pinned down from the euler equation.

The return of portfolio $i$ is approximated by:

$$
r_{i, t+1}=\Delta d_{i, t+1}+\kappa_{0}^{i}+\kappa_{1}^{i} p d_{i, t+1}-p d_{i, t}
$$

where $\kappa_{1}^{i}=\frac{\exp \left(\mu_{i, p d}\right)}{1+\exp \left(\mu_{i, p d}\right)}$ and $\kappa_{0}^{i}=\log \left(1+\exp \left(\mu_{i, p d}\right)\right)-\kappa_{1}^{i} \mu_{i, p d}$ are the approximation constants. Substituting the price-dividend expression into the return approximation yields

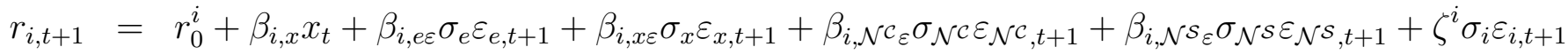

where $r_{0}^{i}=\mu_{i}+\mu_{i, p d}\left(\kappa_{1}^{i}-1\right)+\kappa_{0}^{i}, \beta_{i, x}=\rho \phi_{e} \phi_{x}, \beta_{i, e \varepsilon}=\varphi_{e}^{i}, \beta_{i, x \varepsilon}=\varphi_{x}^{i}+\kappa_{1}^{i} A_{i}, \beta_{i, \mathcal{N}_{\varepsilon} \varepsilon}=\varphi_{\mathcal{N}^{c}}^{i}$ and $\beta_{i, \mathcal{N}^{\mathcal{S}} \varepsilon}=\varphi_{\mathcal{N}^{\mathcal{S}}}^{i}$. All detailed derivations and expressions are in Appendix E.

\subsection{Parameters}

The model is calibrated at monthly frequency, and calibrated parameters are in Table 4. Next, I describe the calibration of the unlevered consumption and preferences, the market portfolio and the sorted portfolios.

Unlevered Consumption Claim and Preferences The long run risk term and preference calibration is based on Bansal and Yaron (2004). The long run term $x_{t}$ is very persistent $\left(\rho_{x}=0.979\right)$ and its innovations are not too volatile $\left(\sigma_{x}=0.0044 \times 0.0078 \approx 3.43 \times 10^{-4}\right)$. Risk aversion is $\gamma=10$ and intertemporal elasticity of substitution is above one $\left(\frac{1}{\rho}=1.5\right)$. 
Table 4: Parameters

\begin{tabular}{|c|c|c|c|}
\hline & Parameters & Value & Target \\
\hline \multicolumn{4}{|c|}{ Factors' Annual Volatility } \\
\hline$\sigma_{e}$ & TFP innovation std. & 0.0124 & Consumption std: $2.5 \%$ \\
\hline$\sigma_{\mathcal{N}^{c}}$ & Concentration innovation std. & 0.0138 & BEA: 0.0055 , Compustat: 0.0757 \\
\hline$\sigma_{\mathcal{N}^{\mathcal{S}}}$ & Sparsity innovation std. & 0.0277 & BEA: 0.0139 , Compustat: 0.0277 \\
\hline \multicolumn{4}{|c|}{ Preferences } \\
\hline$\gamma$ & Risk Aversion & 10 & BY \\
\hline$\frac{1}{0}$ & Elasticity of Intertemporal Substitution & 1.50 & BY \\
\hline$\beta$ & Discount (monthly) & 0.996 & Average Risk-free rate \\
\hline \multicolumn{4}{|c|}{ Technology } \\
\hline$\eta$ & Returns to scale & 0.35 & literature \\
\hline
\end{tabular}

The discount $\beta$ is calibrated to match the average risk-free rate of return at $1.38 \%$ per year observed in the data.

The volatility of the network factors $\left(\sigma_{\mathcal{N}^{c}}\right.$ and $\left.\sigma_{\mathcal{N}^{s}}\right)$ are calibrated to match the standard deviations of the network factors. We can match the volatility of sparsity innovation exactly. The volatility of the innovation in network concentration based on the compustat data is too high (0.0277) and would generate a very volatile consumption process. Using BEA data, volatility of the innovation in network concentration is considerably lower $(0.0055)$. To reconcile these two sources of data and keep the consumption volatility compatile with the data, I set the standard deviaiton of innovation in the network concentration at an intermediate value, $\sigma_{\mathcal{N} c}=0.0138$ (annualized). Finally $\sigma_{e}$ is calibrated to match the consumption growth rate volatility at $2.85 \%$ (annual) exactly, and the returns to scale to capital is set at $\eta=0.35$ from literature.

Market Portfolio Following Bansal and Yaron (2004), the leverage parameter is calibrated at $\phi_{x}^{m}=3.5$, and the parameters of the idiosyncratic component of the market dividend are calibrated at $\varphi_{\xi}^{m}=4.5$, and $\sigma_{\xi}=0.0078$ to generate reasonable dividend growth volatility, average excess return and return volatility. The loading on factors' innovations $\left(\varphi_{V}^{m}, \varphi_{E}^{m}\right.$ and $\left.\varphi_{e}^{m}\right)$ are calibrated to match factor betas of the market portfolio.

Portfolio Dividends The loading on the long-run term are the same as the market portfolio $\left(\phi_{x}^{i}=\phi_{x}^{m}=3.5\right)$ and the loading on its innovation $\left(\varphi_{x}^{i}\right)$ matches the $\beta_{\varepsilon x}$ of the market portfolio precisely. This calibration strategy makes the contribution of the long run risk to 
Table 5: Model Implied Asset Pricing Moments

\begin{tabular}{ccccc} 
Unlevered & Market & \multicolumn{2}{c}{ Sparsity Sorted } & \multicolumn{2}{c}{ Concentration Sorted } \\
Consumption & & $(1)$ & $(2)$ & $(3)$
\end{tabular}

\begin{tabular}{|c|c|c|c|c|c|c|c|c|}
\hline \multicolumn{9}{|c|}{ Panel A: Risk-free return } \\
\hline Mean rf $(\%)$ & - & 1.38 & - & - & - & - & - & - \\
\hline rf vol $(\%)$ & - & 0.39 & - & - & - & - & - & - \\
\hline \multicolumn{9}{|c|}{ Panel B: ERP decomposition } \\
\hline Data (\%) & - & 7.73 & 5.24 & 8.61 & 11.25 & 10.23 & 8.51 & 6.19 \\
\hline Model (\%) & 1.48 & 6.28 & 4.45 & 6.81 & 9.96 & 9.18 & 6.91 & 5.14 \\
\hline$\varepsilon_{e}$ & 0.37 & 0.68 & 0.84 & 0.89 & 2.36 & 1.15 & 0.94 & 2.00 \\
\hline$\varepsilon_{x}$ & 0.70 & 5.36 & 5.36 & 5.36 & 5.36 & 5.36 & 5.36 & 5.36 \\
\hline$\varepsilon_{\mathcal{N} c}$ & 0.19 & 0.08 & 0.04 & 0.41 & 0.13 & 1.99 & 0.49 & -1.91 \\
\hline$\varepsilon_{\mathcal{N}^{\mathcal{S}}}$ & 0.22 & 0.17 & -1.78 & 0.16 & 2.10 & 0.67 & 0.12 & -0.31 \\
\hline \multicolumn{9}{|c|}{ Panel C: Volatility decomposition } \\
\hline Data (\%) & - & 16.89 & 17.60 & 13.78 & 15.13 & 16.18 & 13.60 & 16.27 \\
\hline Model (\%) & 3.21 & 17.56 & 17.56 & 13.34 & 22.34 & 20.29 & 13.56 & 21.27 \\
\hline$\varepsilon_{e}$ & 0.96 & 2.10 & 2.68 & 3.00 & 6.99 & 3.30 & 3.12 & 5.78 \\
\hline$\varepsilon_{x}$ & 0.80 & 7.20 & 7.40 & 7.78 & 6.84 & 6.62 & 7.68 & 6.69 \\
\hline$\varepsilon_{\mathcal{N} c}$ & 0.70 & 0.36 & 0.17 & 1.89 & 0.54 & 7.91 & 2.28 & 7.66 \\
\hline$\varepsilon_{\mathcal{N}^{\mathcal{S}}}$ & 0.75 & 0.66 & 7.30 & 0.67 & 7.97 & 2.46 & 0.49 & 1.14 \\
\hline$\zeta$ & - & 7.24 & 0.00 & 0.00 & 0.00 & 0.00 & 0.00 & 0.00 \\
\hline \multicolumn{9}{|c|}{ Panel D: betas } \\
\hline$\beta_{e}$ & 1.54 & 2.85 & 3.52 & 3.76 & 9.95 & 4.86 & 3.95 & 8.42 \\
\hline$\beta_{x}$ & 13.30 & 101.78 & 101.78 & 101.78 & 101.78 & 101.78 & 101.78 & 101.78 \\
\hline$\beta_{\mathcal{N}^{c}}$ & -1.00 & -0.44 & -0.20 & -2.13 & -0.69 & -10.47 & -2.60 & 10.04 \\
\hline$\beta_{\mathcal{N S}}$ & 0.54 & 0.40 & -4.31 & 0.38 & 5.09 & 1.62 & 0.28 & -0.74 \\
\hline \multicolumn{9}{|c|}{ Panel E: Dividend parameters } \\
\hline$\mu_{i}(\times 100)$ & - & 0.00 & 0.05 & 0.05 & 0.07 & 0.07 & 0.05 & 0.05 \\
\hline$\phi_{x}^{i}$ & - & 3.50 & 3.50 & 3.50 & 3.50 & 3.50 & 3.50 & 3.50 \\
\hline$\varphi_{x}^{i}$ & - & 0.00 & -7.99 & 1.81 & 5.59 & 4.38 & 1.97 & -8.04 \\
\hline$\varphi_{e}^{i}$ & - & 2.85 & 3.52 & 3.76 & 9.95 & 4.86 & 3.95 & 8.42 \\
\hline$\varphi_{\mathcal{N} C}^{i}$ & - & -0.44 & -0.20 & -2.13 & -0.69 & -10.47 & -2.60 & 10.04 \\
\hline$\varphi_{N \mathcal{S}}^{i}$ & - & 0.40 & -4.31 & 0.38 & 5.09 & 1.62 & 0.28 & -0.74 \\
\hline$\zeta^{i}$ & - & 4.50 & 0.00 & 0.00 & 0.00 & 0.00 & 0.00 & 0.00 \\
\hline
\end{tabular}


the risk premium constant across all calibrated portfolio. Thus, all the equity risk premium spreads are due to the other factors, not the long run risk. This guarantees that the long-run risk is quantitatively irrelevant to generate the return spreads observed in the data.

Most importantly, $\varphi_{V}^{i}, \varphi_{E}^{i}$ and $\varphi_{e}^{i}$ are calibrated to match the betas estimated from the data exactly.

\subsection{Calibrated Model}

Table 5 reports asset pricing moments for the market portfolio as well as for the calibrated portfolios. Panel (a) shows the average and volatility of the risk-free rate of return. The average is exactly the same in the data, but the model generates a risk-free rate of return that is not as volatile as in the data: in model its volatility is $0.39 \%$ while in the data it's between $1 \%$ to $2 \%$ depending on the sample considered. Panels (b) and (c) report the risk premium and return volatility in the model and in the data. Finally, panel (d) reports portfolio factor betas, and panel (e) reports the parameterization for each portfolio.

The model replicates the expected return spread in the calibrated portfolios. The model generates a spread of $5.5 \%$ in the sparsity-beta sorted portfolios, while the spread in the data is $6 \%$. The return spread in the concentration-beta sorted portfolio is matched precisely with spread of $-4.04 \%$ both in the data and in the model. The return volatilities are also replicated, except for the third tercile in which the model generates a volatility greater than what is observed in the data.

The contribution of the long run risk factor to the risk premium is constant across all portfolios, which means that the long run risk does not affect the return spread. All the return spread is originated from having different exposure to the factors. Out of the $5.5 \%$ spread in the sparsity-beta sorted portfolios, $70 \%$ (3.88 percentage points) is due different exposures (betas) to innovations in the network sparsity factor. For the concentration-beta sorted portfolios, 97\% (3.90 percentage points out of a total spread of 4.04\%) is due different exposures (betas) to innovations in the network concentration factor. Thus, the driving force behind the return spread is in fact different exposures to innovations in the network factors.

\section{Concluding Remarks}

I have developed a multisector network model predicting that two key characteristics of the network, sparsity and concentration, matter for asset prices and aggregate quantities such as consumption and GDP. Changes in these two factors constitute an aggregate source of risk that is priced in equilibrium. Using return data, there is consistent evidence that 
innovations in the network factors are priced. By sorting stocks on their exposure to factors' innovations and forming portfolios by terciles, there is significant return gap that cannot be explained by standard asset pricing models such the CAPM or the Fama French three factor model. Specifically, sparsity-beta sorted portfolios have a return spread of $6 \%$ per year and concentration-beta sorted portfolios have a return spread of $-4 \%$ per year. A calibrated model successfully replicates these return gaps.

An important extension to this paper is to focus on a less aggregated level, ideally the firm level, in order to endogenize the network formation process. An immediate implication of endogenous network formation is that the equilibrium may become inefficient as firms form their connections ignoring the effects on other firms (network externality). Moreover, endogenous network formation can potentially amplify the effects of network concentration and sparsity depending on firms' incentives to form their connections.

Another appealing extension is to introduce frictions in the credit market. In this case, the standard Long and Plosser (1983) aggregation result no longer holds and sectors' output

won't comove as much for any given network. This extension would allow us to analyze the relation firm level volatility and network characteristics through the lens of an equilibrium model.

\section{References}

Acemoglu, D., V. M. Carvalho, A. Ozdaglar, and A. Tahbaz-Salehi (2012): "The network origins of aggregate fluctuations," Econometrica, 80(5), 1977-2016.

Acemoglu, D., A. Ozdaglar, and A. Tahbaz-Salehi (2013): "Systemic risk and stability in financial networks," Discussion paper, National Bureau of Economic Research.

Ahern, K. (2012): "Network Centrality and the Cross Section of Stock Returns," Available at SSRN 2197370.

Ahern, K., And J. HARford (2010): "The importance of industry links in merger waves," Ross School of Business Paper.

Allen, F., And A. Babus (2008): "Networks in Finance," .

Ang, A., R. J. Hodrick, Y. Xing, And X. Zhang (2006): "The cross-section of volatility and expected returns," The Journal of Finance, 61(1), 259-299.

Aobdia, D., J. Caskey, and N. B. Ozel (2013): "Inter-Industry Network Structure, Information Transfers, and the Cross-Predictability of Earnings and Stock Returns," . 
Atalay, E. (2014): "How Important Are Sectoral Schocks?," .

Babus, A. (2013): "The Formation of Financial Networks," .

Bak, P., K. Chen, J. Scheinkman, and M. Woodford (1993): "Aggregate fluctuations from independent sectoral shocks: self-organized criticality in a model of production and inventory dynamics," Ricerche Economiche, 47(1), 3-30.

Bala, V., And S. Goyal (2000): "A noncooperative model of network formation," Econometrica, 68(5), 1181-1229.

Bansal, R., And A. YARON (2004): "Risks for the long run: A potential resolution of asset pricing puzzles," The Journal of Finance, 59(4), 1481-1509.

Basu, S., J. Fernald, and M. Kimball (2006): "Are Technology Improvements Contractionary?," American Economic Review, 96 (5), 1418-1448.

BELO, F. (2010): "Production-based measures of risk for asset pricing," Journal of Monetary Economics, 57(2), 146-163.

Biggio, S., And J. LA'O (2013): "Financial Frictions in Production Networks," .

Carvalho, V., and X. Gabaix (2013): "The Great Diversification and Its Undoing," American Economic Review, 103(5), 1697-1727.

Carvalho, V. M. (2010): "Aggregate Fluctuations and the Network Structure of Intersectoral Trade," .

(2014): "From Micro to Macro via Production Networks," Journal of Economic Perspectives, 28 (4).

Carvalho, V. M., and B. Grassi (2014): "Firm Dynamics and the Granular Hypothesis,"

Carvalho, V. M., and N. Voigtlander (2014): "Input Diffusion and the Evolution of Production Networks," .

Cohen, L., And A. Frazzini (2008): "Economic links and predictable returns," The Journal of Finance, 63(4), 1977-2011.

Cohen, L., A. Frazzini, and C. Malloy (2008): "The Small World of Investing: Board Connections and Mutual Fund Returns," Journal of Political Economy, 116(5), 951-979. 
FARBOODI, M. (2014): "Intermediation and Voluntary Exposure to Counterarty Risk," .

Fernald, J. (2012): "A Quarterly, Utilization-Adjusted Series on Total Factor Productivity," FRBSF Working Paper 2012-19.

Fernald, J., And K. Matoba (2009): "Growth Accounting, Potential Output, and the Current Recession," FRBSF Economic Letter 2009-26.

Gabaix, X. (2011): "The granular origins of aggregate fluctuations," Econometrica, 79(3), $733-772$.

Galeotti, A., and S. Goyal (2010): "The law of the few," The American Economic Review, pp. 1468-1492.

Gomes, J., L. Kogan, and M. Yogo (2009): "Durability of Output and Expected Stock Returns," Journal of Political Economy, 117(5), 941-986.

Gomes, J., And L. Schmid (2010): "Equilibrium credit spreads and the macroeconomy," The Wharton School Research Paper, (42).

Herskovic, B., B. T. Kelly, H. Lustig, and S. Van Nieuwerburgh (2014): "The Common Factor in Idiosyncratic Volatility: Quantitative Asset Pricing Implications," Discussion paper, National Bureau of Economic Research.

Herskovic, B., And J. Ramos (2014): "Network Formation and Information Acquisition,"

Hou, K., And D. T. Robinson (2006): "Industry concentration and average stock returns," The Journal of Finance, 61(4), 1927-1956.

Jermann, U. (2013): "A production-based model for the term structure," Discussion paper, National Bureau of Economic Research.

Jermann, U. J. (1998): "Asset pricing in production economies," Journal of Monetary Economics, 41(2), 257-275.

- (2010): "The equity premium implied by production," Journal of Financial Economics, 98(2), 279-296.

Jovanovic, B. (1987): "Micro shocks and aggregate risk," The Quarterly Journal of Economics, 102(2), 395-409. 
KATz, L. (1953): "A new status index derived from sociometric analysis," Psychometrika, 18(1), 39-43.

Kelly, B., H. Lustig, and S. Van Nieuwerburgh (2013): "Firm Volatility in Granular Networks," Discussion paper, National Bureau of Economic Research.

Kogan, L., D. Papanikolaou, and N. Stoffman (2013): "Technological innovation: Winners and losers," Discussion paper, National Bureau of Economic Research.

Kuehn, L.-A. (2009): "Disentangling investment returns and stock returns: The importance of time-to-build," .

Kuehn, L.-A., And L. Schmid (2011): "Investment-based corporate bond pricing," Manuscript, Duke University.

Kung, H., And L. Schmid (2011): "Innovation, growth and asset prices," .

Lochstoer, L. A. (2009): "Expected returns and the business cycle: Heterogeneous goods and time-varying risk aversion," Review of Financial Studies, 22(12), 5251-5294.

Long, J. B., And C. I. Plosser (1983): "Real business cycles," The Journal of Political Economy, pp. 39-69.

Loualiche, E. (2012): “Asset Pricing with Entry and Imperfect Competition Job Market Paper," .

Malamud, S., and M. Rostek (2014): "Decentralized Exchange," .

Malloy, C. J., T. J. Moskowitz, and A. Vissing-Jørgensen (2009): "Long-Run Stockholder Consumption Risk and Asset Returns," The Journal of Finance, 64(6), 24272479 .

OBERFIELD, E. (2013): "Business networks, production chains, and productivity: A theory of input-output architecture," .

Papanikolaou, D. (2011): "Investment Shocks and Asset Prices," Journal of Political Economy, 119 (4), 639-685.

van Binsbergen, J. H. (2007): "Good-specific habit formation and the cross-section of expected returns," in AFA 2009 San Francisco Meetings Paper.

Yogo, M. (2006): "A Consumption-Based Explanation of Expected Stock Returns," The Journal of Finance, 61(2), 539-580. 


\section{Appendix}

\section{A Solving firms and household problems}

Firms Firms maximize per-period dividend:

$$
\begin{aligned}
D_{i, t}=\max _{\left\{y_{i j, t}\right\}_{j}, I_{i, t}} & P_{i, t} \varepsilon_{i, t} I_{i, t}^{\eta}-\sum_{j=1}^{n} P_{j, t} y_{i j, t} \\
\text { s.t. } & {\left[\sum_{j=1}^{n} w_{i j} y_{i j, t}^{1-1 / \nu}\right]^{\frac{1}{1-1 / \nu}}-I_{i, t}=0 \quad\left(\mu_{i, t}\right) }
\end{aligned}
$$

where $\nu$ is the elasticity of substitution between two distinct inputs, $\eta \in(0,1)$ is the returns to scale, and $\mu_{i, t}$ is the Lagrange multiplier.

Hence the FOC are:

$$
\begin{aligned}
y_{i j, t}: \quad-P_{j, t}+\tilde{\mu}_{i, t} I_{i, t}^{1 / \nu} y_{i j, t}^{-1 / \nu} w_{i j}=0 & \Longrightarrow y_{i j, t}=\mu_{i, t}^{\nu} \frac{w_{i j}^{\nu} I_{i, t}}{P_{j, t}^{\nu}} \\
I_{i, t}: \quad-\mu_{i, t}+\eta P_{i, t} \varepsilon_{i, t} I_{i, t}^{\eta-1}=0 & \Longrightarrow I_{i, t}=\left(\frac{\eta P_{i, t} \varepsilon_{i, t}}{\mu_{i, t}}\right)^{\frac{1}{1-\eta}}
\end{aligned}
$$

and the remaining Karush-Kuhn-Tucker condition is:

$$
\left[\sum_{j=1}^{n} w_{i j} y_{i j, t}^{1-1 / \nu}\right]^{\frac{1}{1-1 / \nu}}-I_{i, t}=0
$$

These are the FOC order condition of the firm's problem. Let's simplify them a bit more. First, let's substitution the $y_{i j, t}$ FOC into the $I_{i, t}$ definition: 


$$
\begin{aligned}
I_{i, t} & =\left[\sum_{j=1}^{n} w_{i j} y_{i j, t}^{1-1 / \nu}\right]^{\frac{1}{1-1 / \nu}} \\
I_{i, t} & =\left[\sum_{j=1}^{n} w_{i j}\left(\left(\mu_{i, t}\right)^{\nu} \frac{w_{i j}^{\nu} I_{i, t}}{P_{j, t}^{\nu}}\right)^{1-1 / \nu}\right]^{\frac{1}{1-1 / \nu}} \\
1 & \left.=\left(\mu_{i, t}\right)^{\nu}\left[\sum_{j=1}^{n} w_{i j}\left(\frac{w_{i j}^{\nu}}{P_{j, t}^{\nu}}\right)^{1-1 / \nu}\right]^{\frac{1}{1-1 / \nu}}\right]^{-\frac{1 / \nu}{1-1 / \nu}}=\left[\sum_{j=1}^{n} w_{i j}^{\nu} P_{j, t}^{1-\nu}\right]^{\frac{1}{1-\nu}} \\
\therefore \mu_{i, t} & =\left[\sum_{j=1}^{n} w_{i j}\left(\frac{w_{i j}^{\nu}}{P_{j, t}^{\nu}}\right)^{1-1 / \nu}\right]^{1-1}
\end{aligned}
$$

For $\nu=1$, we have $\mu_{i, t}=\frac{\prod_{j=1}^{n} P_{j, t}^{w_{i j}}}{\prod_{j=1}^{n} w_{i j}^{w_{i j}}} . \mu_{i, t}$ is a network-weighted average of spot market prices and it has to be equal to the market value value of one extra unit of investment, according to the investment FOC. Hence, firms' problem is fully solved by:

$$
\begin{aligned}
y_{i j, t} & =\mu_{i, t}^{\nu} \frac{w_{i j}^{\nu} I_{i, t}}{P_{j, t}^{\nu}} \\
I_{i, t} & =\left(\frac{\eta P_{i, t} \varepsilon_{i, t}}{\mu_{i, t}}\right)^{\frac{1}{1-\eta}} \\
\mu_{i, t} & =\left[\sum_{j=1}^{n} w_{i j}^{\nu} P_{j, t}^{1-\nu}\right]^{\frac{1}{1-\nu}}
\end{aligned}
$$

Household Let $\lambda_{t}$ be the Lagrange multiplier for the period $t$ budget constraint. Then, the FOC for $c_{i, 1}$ is given by

$$
J_{t}^{\rho}(1-\beta) \mathcal{C}_{t}^{-\rho} \frac{\partial \mathcal{C}_{t}}{\partial c_{i, t}}=P_{i, t} \lambda_{t}
$$

which implies

$$
\frac{\frac{\partial \mathcal{C}_{t}}{\partial c_{i, t}}}{\frac{\partial \mathcal{C}_{t}}{\partial c_{j, t}}}=\frac{P_{i, t}}{P_{j, t}}
$$

and it represents the intra-period consumption allocation. For Cobb-Douglas aggregator, the consumption allocation as a function of spot market prices: 


$$
c_{i, t}=\alpha_{i} \frac{\sum_{i=1}^{n} D_{i, t}}{P_{i, t}}
$$

For the inter-temporal consumption allocation, the FOC for $\varphi_{i, t+1}$ is

$$
J_{t}^{\rho} \beta \mathbb{E}_{t}\left[J_{t+1}^{1-\gamma}\right]^{\frac{\gamma-\rho}{1-\gamma}} \mathbb{E}_{t}\left[J_{t+1}^{\gamma} \frac{\partial J_{t+1}}{\partial \varphi_{i, t+1}}\right]=\lambda_{t} Q_{i, t}
$$

Applying the envelope theorem:

$$
\begin{aligned}
\frac{\partial J_{t}}{\partial \varphi_{i, t}} & =\lambda_{t}\left(D_{i, t}+Q_{i, t}\right) \\
& =J_{t}^{\rho}(1-\beta) \mathcal{C}_{t}^{-\rho} \frac{1}{P_{1, t}} \frac{\partial \mathcal{C}_{t}}{\partial c_{1, t}}\left(D_{i, t}+Q_{i, t}\right)
\end{aligned}
$$

Evaluating this equation one period ahead and substituting in the inter-temporal FOC yields

$J_{t}^{\rho} \beta \mathbb{E}_{t}\left[J_{t+1}^{1-\gamma}\right]^{\frac{\gamma-\rho}{1-\gamma}} \mathbb{E}_{t}\left[J_{t+1}^{\gamma} J_{t+1}^{\rho}(1-\beta) \mathcal{C}_{t+1}^{-\rho} \frac{1}{P_{1, t+1}} \frac{\partial \mathcal{C}_{t+1}}{\partial c_{1, t+1}}\left(D_{i, t+1}+Q_{i, t+1}\right)\right]=J_{t}^{\rho}(1-\beta) \mathcal{C}_{t}^{-\rho} \frac{1}{P_{1, t}} \frac{\partial \mathcal{C}_{t}}{\partial c_{1, t}} Q_{i, t}$

which can be further simplified and rearranged as

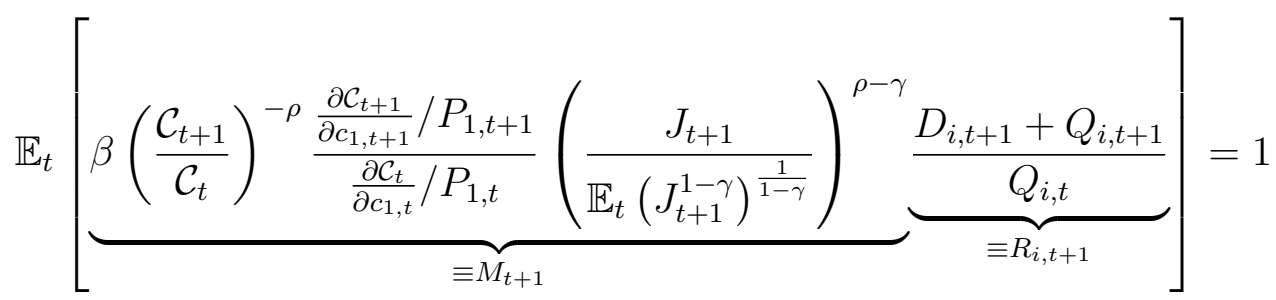

\section{B Price Normalization}

\section{B.1 Simplifying the Stochastic Discount Factor}

I'll choose the price normalization in order to simply the SDF expression. Specifically, prices will be normalized such that the marginal aggregtator term is set to 1 :

$$
\frac{\partial \mathcal{C}_{t}}{\partial c_{i, t}} / P_{i, t}=1 \quad \forall i, t
$$

It turns out that the necessary price normalization has a very intuitive interpretation: it makes the risk-free rate of return the return on a claim to the aggregator utility. To find the necessary price normalization, I'll write marginal aggregtator element of the SDF in terms 
of spot market prices:

$$
\begin{aligned}
\frac{\partial \mathcal{C}_{t}}{\partial c_{i, t}} / P_{i, t} & =\frac{\alpha_{i} \mathcal{C}_{t}}{P_{i, t} c_{i, t}} \\
& =\frac{\alpha_{i}}{P_{i, t}} \prod_{j=1}^{n}\left(\frac{c_{j, t}}{c_{i, t}}\right)^{\alpha_{j}} \\
& =\frac{\alpha_{i}}{P_{i, t}} \prod_{j=1}^{n}\left(\frac{\alpha_{j} / P_{j, t}}{\alpha_{i} / P_{i, t}}\right)^{\alpha_{j}} \\
& =\prod_{j=1}^{n}\left(\frac{\alpha_{j}}{P_{j, t}}\right)^{\alpha_{j}}
\end{aligned}
$$

Therefore, the prices are normalized such that:

$$
\prod_{j=1}^{n} P_{j, t}^{\alpha_{j}}=\prod_{j=1}^{n} \alpha_{j}^{\alpha_{j}}
$$

\section{B.2 Price Normalization and Returns}

The risk-free rate of return is given by

$$
R_{r f, t}=\frac{1}{\mathbb{E}_{t}\left[M_{t+1}\right]}
$$

The risk-free rate of return is sensitive to the price normalization chosen. For example, if good 1 is defined as the numeraire, then the "risk free" rate of return is the return on a claim to one unit of good 1 . Since all relative prices may change next period, this claim is still risky as one unit of good 1 may buy different units of other goods. Hence, when the household buy such claim, its payoff (in terms of aggregator utility) is random, because he will substitute consumption goods in order to maximize utility.

To avoid this issue, I'm going to define the risk-free rate as the return on a "risk-less" bundle, specifically a bundle that cancels this substitution effect keeping the aggregator utility constant. Hence, I'm defining the risk-free return as the return on a claim to the aggregator utility. This bundle has satisfy the intra-period first order condition of the representative agent and its price has to be normalized to 1 . We can define such risk-less claim as

Definition 1 (Risk-less Claim). The risk-less claim is a claim to the following bundle:

$$
\left(\frac{\alpha_{1}}{P_{1, t}}, \ldots, \frac{\alpha_{n}}{P_{n, t}}\right)
$$


The price of such claim is 1 , because $\sum_{j=1}^{n} \alpha_{j}=1$. The risk-less claim satisfies the intraperiod first order condition and is a claim to the consumption aggregator: buying $\kappa$ units of such claim yields the following consumption aggregator amount:

$$
\mathcal{C}_{t}=\kappa \prod_{j=1}^{n}\left(\frac{\alpha_{j}}{P_{j, t}}\right)^{\alpha_{j}}=\kappa
$$

\section{B.3 Consumption expenditure and utility aggregator}

Another useful property of the price normalization chosen is that consumption expenditure and utility aggregator are the same in equilibrium. Evaluating the consumption aggregator in equilibrium at the normalized prices yields

$$
\begin{aligned}
\mathcal{C}\left(c_{t}\right) & =\prod_{j=1}^{n} c_{j, t}^{\alpha_{j}} \\
& =\prod_{j=1}^{n}\left(\frac{\alpha_{j} \omega_{t}}{P_{j, t}}\right)^{\alpha_{j}} \\
& =\omega_{t} \prod_{j=1}^{n}\left(\frac{\alpha_{j}}{P_{j, t}}\right)^{\alpha_{j}} \\
& =\omega_{t}
\end{aligned}
$$

\section{Proof of Lemma 1}

Proof. This is a standard proof of Epstein-Zin SDF. However, it's not exactly the same, because we have to keep track of the extras terms coming from the consumption aggregator.

Total wealth is defined as

$$
\mathcal{W}_{t}=\omega_{t}+\mathbb{E}_{t}\left[M_{t+1} \mathcal{W}_{t+1}\right]
$$

As long as the consumption aggregator $\mathcal{C}(\cdot)$ is homogeneous of degree one, one can guess and verify that total period $t$ wealth $\left(\mathcal{W}_{t}\right)$ in terms of good 1 can be written as

$$
\frac{\mathcal{W}_{t}}{P_{1, t}}=\left(\frac{\partial \log \left(U_{t}\right)}{\partial c_{1, t}}\right)^{-1}=\frac{U_{t}}{\frac{\partial U_{t}}{\partial c_{1, t}}}
$$

where RHS is evaluated at the household solution. Before verifying the guess, some useful expressions will be derived. First, using any aggregator $\mathcal{C}(\cdot)$ homogeneous of degree one and using the fact that the ration of the marginal aggregator has to equal the relative prices, 
we may write the total period $t$ consumption expenditure $\left(\omega_{t}\right)$ in term of the consumption aggregator:

$$
\begin{aligned}
\frac{\frac{\partial \mathcal{C}_{t}}{\partial c_{i, t}}}{\frac{\partial \mathcal{C}_{t}}{\partial c_{1, t}}} & =\frac{P_{i, t}}{P_{1, t}} \\
c_{i, t} \frac{\partial \mathcal{C}_{t}}{\partial c_{i, t}} & =\frac{1}{P_{1, t}} \frac{\partial \mathcal{C}_{t}}{\partial c_{1, t}} c_{i, t} P_{i, t} \\
\sum_{i=1}^{n} c_{i, t} \frac{\partial \mathcal{C}_{t}}{\partial c_{i, t}} & =\frac{1}{P_{1, t}} \frac{\partial \mathcal{C}_{t}}{\partial c_{1, t}} \sum_{i=1}^{n} c_{i, t} P_{i, t} \\
\mathcal{C}_{t} & =\frac{1}{P_{1, t}} \frac{\partial \mathcal{C}_{t}}{\partial c_{1, t}} \sum_{i=1}^{n} c_{i, t} P_{i, t}
\end{aligned}
$$

In the last step, I used that $\mathcal{C}_{t}$ is homogeneous of degree one. Rearranging the above expression, the consumption expenditure in terms of good 1 can be expressed as:

$$
\frac{\omega_{t}}{P_{1, t}} \equiv \frac{\sum_{i=1}^{n} P_{i, t} c_{i, t}}{P_{1, t}}=\frac{\mathcal{C}_{t}}{\partial \mathcal{C}_{t} / \partial c_{1, t}}
$$

Second, from the household FOC, the guess in equation 20 can be written as:

$$
\frac{\mathcal{W}_{t}}{P_{1, t}}=\frac{J_{t}^{1-\rho}}{\mathcal{C}_{t}^{-\rho}(1-\beta) \partial \mathcal{C}_{t} / \partial c_{1, t}}
$$

To verify the initial guess equation, one can start from the total wealth definition evalu- 
ated at the optimal

$$
\begin{aligned}
& \mathcal{W}_{t} \quad=\omega_{t}+\mathbb{E}_{t}\left[M_{t+1} \mathcal{W}_{t+1}\right] \\
& \frac{J_{t}^{1-\rho}}{\mathcal{C}_{t}^{-\rho}(1-\beta) \frac{1}{P_{1, t}} \partial \mathcal{C}_{t} / \partial c_{1, t}}=\frac{\mathcal{C}_{t}}{\frac{1}{P_{1, t}} \partial \mathcal{C}_{t} / \partial c_{1, t}}+\mathbb{E}_{t}\left[M_{t+1} \frac{J_{t+1}^{1-\rho}}{\mathcal{C}_{t+1}^{-\rho}(1-\beta) \frac{1}{P_{1, t+1}} \partial \mathcal{C}_{t+1} / \partial c_{1, t+1}}\right] \\
& \frac{J_{t}^{1-\rho}}{\mathcal{C}_{t}^{-\rho}(1-\beta) \partial \mathcal{C}_{t} / \partial c_{1, t}}=\frac{\mathcal{C}_{t}}{\partial \mathcal{C}_{t} / \partial c_{1, t}}+\mathbb{E}_{t}\left[\beta \frac{\mathcal{C}_{t+1}^{-\rho} \frac{\partial \mathcal{C}_{t+1}}{\partial c_{1, t+1}}}{\mathcal{C}_{t}^{-\rho} \frac{\partial \mathcal{C}_{t}}{\partial c_{1, t}}}\left(\frac{J_{t+1}}{\mathbb{E}_{t}\left(J_{t+1}^{1-\gamma}\right)^{\frac{1}{1-\gamma}}}\right)^{\rho-\gamma} \frac{J_{t+1}^{1-\rho}}{\mathcal{C}_{t+1}^{-\rho}(1-\beta) \partial \mathcal{C}_{t+1} / \partial c_{1, t+1}}\right] \\
& \frac{J_{t}^{1-\rho}}{\mathcal{C}_{t}^{-\rho}(1-\beta) \partial \mathcal{C}_{t} / \partial c_{1, t}}=\frac{\mathcal{C}_{t}}{\partial \mathcal{C}_{t} / \partial c_{1, t}}+\mathbb{E}_{t}\left[\beta \frac{1}{\mathcal{C}_{t}^{-\rho} \frac{\partial \mathcal{C}_{t}}{\partial c_{1, t}}}\left(\frac{J_{t+1}}{\mathbb{E}_{t}\left(J_{t+1}^{1-\gamma}\right)^{1-\gamma}}\right)^{\rho-\gamma} \frac{J_{t+1}^{1-\rho}}{(1-\beta)}\right] \\
& \frac{J_{t}^{1-\rho}}{\mathcal{C}_{t}^{-\rho}(1-\beta)} \quad=\mathcal{C}_{t}+\mathbb{E}_{t}\left[\beta \frac{\mathcal{C}_{t+1}^{-\rho}}{\mathcal{C}_{t}^{-\rho}}\left(\frac{J_{t+1}}{\mathbb{E}_{t}\left(J_{t+1}^{1-\gamma}\right)^{\frac{1}{1-\gamma}}}\right)^{\rho-\gamma} \frac{J_{t+1}^{1-\rho}}{\mathcal{C}_{t+1}^{-\rho}(1-\beta)}\right] \\
& J_{t}^{1-\rho} \quad=(1-\beta) \mathcal{C}_{t}^{1-\rho}+\mathbb{E}_{t}\left[\beta\left(\frac{J_{t+1}}{\mathbb{E}_{t}\left(J_{t+1}^{1-\gamma}\right)^{\frac{1}{1-\gamma}}}\right)^{\rho-\gamma} J_{t+1}^{1-\rho}\right] \\
& J_{t}^{1-\rho} \quad=(1-\beta) \mathcal{C}_{t}^{1-\rho}+\mathbb{E}_{t}\left[\beta \frac{J_{t+1}^{1-\gamma}}{\mathbb{E}_{t}\left(J_{t+1}^{1-\gamma}\right)^{\frac{\rho-\gamma}{1-\gamma}}}\right] \\
& J_{t}^{1-\rho} \quad=(1-\beta) \mathcal{C}_{t}^{1-\rho}+\beta \mathbb{E}_{t}\left(J_{t+1}^{1-\gamma}\right)^{1-\frac{\rho-\gamma}{1-\gamma}} \\
& J_{t} \quad=\left[(1-\beta) \mathcal{C}_{t}^{1-\rho}+\beta \mathbb{E}_{t}\left(J_{t+1}^{1-\gamma}\right)^{\frac{1-\rho}{1-\gamma}}\right]^{\frac{1}{1-\rho}}
\end{aligned}
$$

Using the EZ preference definition, the expected value term in the SDF can written as:

$$
\mathbb{E}_{t}\left(U_{t+1}^{1-\gamma}\right)^{\frac{1}{1-\gamma}}=\beta^{\frac{-1}{1-\rho}}\left[U_{t}^{1-\rho}-(1-\beta)\left(\mathcal{C}_{t}\right)^{1-\rho}\right]^{\frac{1}{1-\rho}}
$$

and the value function itself can be written in terms of wealth:

$$
\frac{\mathcal{W}_{t}}{P_{1, t}}=\frac{J_{t}^{1-\rho}}{\mathcal{C}_{t}^{-\rho}(1-\beta) \partial \mathcal{C}_{t} / \partial c_{1, t}} \Longrightarrow J_{t}=\left[\mathcal{W}_{t} \mathcal{C}_{t}^{-\rho}(1-\beta) \frac{1}{P_{1, t}} \partial \mathcal{C}_{t} / \partial c_{1, t}\right]^{\frac{1}{1-\rho}}
$$


Next, the SDF in terms of wealth return:

$$
\begin{aligned}
& M_{t+1} \equiv \beta \frac{\mathcal{C}_{t+1}^{-\rho} \frac{1}{P_{1, t+1}} \frac{\partial \mathcal{C}_{t+1}}{\partial c_{1, t+1}}}{\mathcal{C}_{t}^{-\rho} \frac{1}{P_{1, t}} \partial \frac{\partial \mathcal{C}_{t}}{\partial c_{1, t}}}\left(\frac{J_{t+1}}{\mathbb{E}_{t}\left(J_{t+1}^{1-\gamma}\right)^{\frac{1}{1-\gamma}}}\right)^{\rho-\gamma} \\
& =\beta \frac{\mathcal{C}_{t+1}^{-\rho} \frac{1}{P_{1, t+1}} \frac{\partial \mathcal{C}_{t+1}}{\partial c_{1, t+1}}}{\mathcal{C}_{t}^{-\rho} \frac{1}{P_{1, t}} \frac{\partial C_{t}}{\partial c_{1, t}}}\left(\frac{J_{t+1}}{\beta^{\frac{-1}{1-\rho}}\left[J_{t}^{1-\rho}-(1-\beta)\left(\mathcal{C}_{t}\right)^{1-\rho}\right]^{\frac{1}{1-\rho}}}\right)^{\rho-\gamma} \\
& =\beta \frac{\mathcal{C}_{t+1}^{-\rho} \frac{1}{P_{1, t+1}} \frac{\partial \mathcal{C}_{t+1}}{\partial \mathcal{C}_{1, t+1}}}{\mathcal{C}_{t}^{-\rho} \frac{1}{P_{1, t}} \partial \frac{\partial \mathcal{C}_{t}}{\partial c_{1, t}}}\left(\frac{\left[\mathcal{W}_{t+1} \mathcal{C}_{t+1}^{-\rho}(1-\beta) \frac{1}{P_{1, t+1}} \partial \mathcal{C}_{t+1} / \partial c_{1, t+1}\right]^{\frac{1}{1-\rho}}}{\beta^{\frac{-1}{1-\rho}}\left[\mathcal{W}_{t} \mathcal{C}_{t}^{-\rho}(1-\beta) \frac{1}{P_{1, t}} \partial \mathcal{C}_{t} / \partial c_{1, t}-(1-\beta)\left(\mathcal{C}_{t}\right)^{1-\rho}\right]^{\frac{1}{1-\rho}}}\right)^{\rho-\gamma} \\
& =\beta \frac{\mathcal{C}_{t+1}^{-\rho} \frac{1}{P_{1, t+1}} \frac{\partial \mathcal{C}_{t+1}}{\partial c_{1, t+1}}}{\mathcal{C}_{t}^{-\rho} \frac{1}{P_{1, t}} \frac{\partial \mathcal{C}_{t}}{\partial c_{1, t}}}\left(\frac{\beta \mathcal{W}_{t+1} \mathcal{C}_{t+1}^{-\rho} \frac{1}{P_{1, t+1}} \partial \mathcal{C}_{t+1} / \partial c_{1, t+1}}{\mathcal{W}_{t} \mathcal{C}_{t}^{-\rho} \frac{1}{P_{1, t}} \partial \mathcal{C}_{t} / \partial c_{1, t}-\left(\mathcal{C}_{t}\right)^{1-\rho}}\right)^{\frac{\rho-\gamma}{1-\rho}} \\
& =\beta^{\frac{1-\gamma}{1-\rho}}\left(\frac{\mathcal{C}_{t+1}}{\mathcal{C}_{t}}\right)^{-\frac{1-\gamma}{1-\rho}}\left(\frac{\frac{1}{P_{1, t+1}} \partial \mathcal{C}_{t+1} / \partial c_{1, t+1}}{\frac{1}{P_{1, t}} \partial \mathcal{C}_{t} / \partial c_{1, t}}\right)^{\frac{1-\gamma}{1-\rho}}\left(\frac{\mathcal{W}_{t+1}}{\mathcal{W}_{t}-\frac{1}{P_{1, t}} \partial \mathcal{C}_{t} / \partial c_{1, t}}\right)^{\frac{\rho-\gamma}{1-\rho}} \\
& =\beta^{\theta}\left(\frac{\mathcal{C}_{t+1}}{\mathcal{C}_{t}}\right)^{-\rho \theta}\left(\frac{\frac{1}{P_{1, t+1}} \partial \mathcal{C}_{t+1} / \partial c_{1, t+1}}{\frac{1}{P_{1, t}} \partial \mathcal{C}_{t} / \partial c_{1, t}}\right)^{\theta}\left(\frac{\mathcal{W}_{t+1}}{\mathcal{W}_{t}-\omega_{t}}\right)^{1-\theta} \\
& =\beta^{\theta}\left(\frac{\mathcal{C}_{t+1}}{\mathcal{C}_{t}}\right)^{-\rho \theta}\left(\frac{\frac{1}{P_{1, t+1}} \partial \mathcal{C}_{t+1} / \partial c_{1, t+1}}{\frac{1}{P_{1, t}} \partial \mathcal{C}_{t} / \partial c_{1, t}}\right)^{\theta}\left(R_{t+1}^{\mathcal{W}}\right)^{1-\theta} \\
& =\beta^{\theta}\left(\frac{\omega_{t+1}}{\omega_{t}}\right)^{-\rho \theta}\left(\frac{\frac{1}{P_{1, t+1}} \partial \mathcal{C}_{t+1} / \partial c_{1, t+1}}{\frac{1}{P_{1, t}} \partial \mathcal{C}_{t} / \partial c_{1, t}}\right)^{1-\gamma}\left(R_{t+1}^{\mathcal{W}}\right)^{1-\theta}
\end{aligned}
$$

where $R_{t+1}^{\mathcal{W}}=\frac{\mathcal{W}_{t+1}}{\mathcal{W}_{t}-\omega_{t}}$ it the return on total wealth and $\theta=\frac{1-\gamma}{1-\rho}$. 


\section{Closed-form expressions: derivations}

\section{D.1 Output share}

Starting form the market clearing condition for good $i$, we have:

$$
\begin{aligned}
c_{i, t}+\sum_{j} y_{j i, t} & =Y_{i, t} \\
c_{i, t}+\sum_{j} \mu_{i, t}^{\nu} \frac{w_{j i, t}^{\nu} I_{j, t}}{P_{i, t}^{\nu}} & =Y_{i, t} \\
P_{i, t} c_{i, t}+\sum_{j} \mu_{j}^{\nu} w_{j i, t}^{\nu} P_{i, t}^{1-\nu} I_{j, t} & =P_{i, t} Y_{i, t} \\
\alpha_{i}(1-\eta) z_{t}+\eta \sum_{j} w_{j i, t}^{\nu}\left(\frac{P_{i, t}}{\mu_{j}}\right)^{1-\nu} P_{j, t} Y_{j, t} & =P_{i, t} Y_{i, t} \\
\alpha_{i}(1-\eta) z_{t}+\eta \sum_{j} \frac{w_{j i, t}^{\nu} P_{i, t}^{1-\nu}}{\sum_{s} w_{j s}^{\nu} P_{s}^{1-\nu}} P_{j, t} Y_{j, t} & =P_{i, t} Y_{i, t} \\
(1-\eta) \alpha_{i} z_{t}+\eta \sum_{j} P_{j, t} Y_{j, t} \tilde{w}_{j i, t} & =P_{i, t} Y_{i, t},
\end{aligned}
$$

where $z_{t}=\sum_{i} P_{i, t} Y_{i, t}$ is the total output of the economy at period $t$ and $\tilde{w}_{j i, t}=\frac{w_{j i, t}^{\nu} P_{i, t}^{1-\nu}}{\sum_{s} w_{j s}^{\nu} P_{s}^{1-\nu}}$.

In matrix notation, the above system becomes:

$$
(1-\eta) \alpha z_{t}+\eta \tilde{W}_{t}^{\prime} \overrightarrow{P Y}_{t}=\overrightarrow{P Y}_{t}
$$

where $\overrightarrow{P Y}_{t}$ is a column vector of sectors' output, $\tilde{W}_{t}$ is a $n \times n$ matrix whose $(\mathrm{i}, \mathrm{j})$ entry is $\tilde{w}_{j i, t}$ and $\alpha$ is a column vector with preference weights.

We can solve system 23 and express the output of each firm as a fraction of the total output.

$$
\overrightarrow{P Y}_{t}=\underbrace{(1-\eta)\left[\mathbb{I}-\eta \tilde{W}_{t}^{\prime}\right]^{-1} \alpha}_{\equiv \delta_{t}} z=\delta_{t} z_{t}
$$

where $\delta_{t}$ is a $n \times 1$ vector of output shares of each sector/firm.

When $\nu=1$, the output shares is completely determined by the network and household preferences:

$$
\delta_{t}=(1-\eta)\left[\mathbb{I}-\eta W_{t}^{\prime}\right]^{-1} \alpha
$$




\section{D.2 Equilibrium Conditions}

The stochastic discount factor, however, depends on changes in the aggregate consump-

tion expenditure, namely $\log \left(\frac{\omega_{t+1}}{\omega_{t}}\right)$. Consumption expenditure is proportional to the total output, as

$$
\omega_{t}=\sum_{j} c_{j, t} P_{j, t}=\sum_{j} D_{j, t}=(1-\eta) \sum_{j} P_{j, t} Y_{j, t}=(1-\eta) z_{t} .
$$

This implies that total output growth is equal to total consumption expenditure growth:

$$
\log \left(\frac{\omega_{t+1}}{\omega_{t}}\right)=\log \left(\frac{z_{t+1}}{z_{t}}\right)
$$

Using the first order condition of firm $i$, we can derive equation 15 as follows:

$$
\begin{aligned}
\delta_{i, t} z_{t} & =P_{i, t} Y_{i, t} \\
\delta_{i, t} z_{t} & =\frac{\mu_{i, t} I_{i, t}}{\eta} \\
\delta_{i, t} z_{t} & =\frac{\mu_{i, t}\left(\frac{\eta P_{i, t} \varepsilon_{i, t}}{\mu_{i, t}}\right)^{\frac{1}{1-\eta}}}{\eta} \\
\delta_{i, t} z_{t} & =\mu_{i, t}^{-\frac{\eta}{1-\eta}} P_{i, t}^{\frac{1}{1-\eta}} \varepsilon_{i, t}^{\frac{1}{1-\eta}} \eta^{\frac{\eta}{1-\eta}} \\
\left(\delta_{i, t} z_{t}\right)^{1-\eta} & =\mu_{i, t}^{-\eta} P_{i, t} \varepsilon_{i, t} \eta^{\eta}
\end{aligned}
$$

\section{D.3 Output growth: Cobb-Doublas case}

Assuming that $\nu=1$, the Langrange multiplier becomes:

$$
\mu_{i, t}=\prod_{j} \frac{P_{j, t}^{w_{i j, t}}}{w_{i j, t}^{w_{i j, t}}}
$$

Substituting the multiplier into (15):

$$
\begin{aligned}
\left(\delta_{i, t} z_{t}\right)^{1-\eta} & =\mu_{i, t}^{-\eta} P_{i, t} \varepsilon_{i, t} \eta^{\eta} \\
(1-\eta) \log \delta_{i, t}+(1-\eta) \log z_{t} & =-\eta \log \mu_{i, t}+\log P_{i, t}+\log \varepsilon_{i, t}+\eta \log \eta \\
(1-\eta) \log \delta_{i, t}+(1-\eta) \log z_{t} & =-\eta \sum_{j} w_{i j, t} \log P_{j, t}+\eta \sum_{j} w_{i j, t} \log w_{i j, t}+\log P_{i, t}+\log \varepsilon_{i, t}+\eta \log \eta
\end{aligned}
$$

Writing the above system in matrix notation and using the price normalization yields: 


$$
\begin{aligned}
(1-\eta) \log \delta_{t}+(1-\eta) \mathbf{1} \log z_{t} & =-\eta W \log P_{t}+\eta \overrightarrow{\mathcal{N}^{\mathcal{S}}}{ }_{t}+\log P_{t}+\log \varepsilon_{t}+\mathbf{1} \eta \log \eta \\
\log P_{t} & =[\mathrm{I}-\eta W]^{-1}\left((1-\eta) \log \delta_{t}+(1-\eta) \mathbf{1} \log z_{t}-\eta \overrightarrow{\mathcal{N}}_{t}-\log \varepsilon_{t}-\mathbf{1} \eta \log \eta\right) \\
\alpha^{\prime} \log P_{t} & =\alpha^{\prime}[\mathrm{I}-\eta W]^{-1}\left((1-\eta) \log \delta_{t}+(1-\eta) \log z_{t}-\eta \overrightarrow{\mathcal{N}^{\mathcal{s}}}{ }_{t}-\log \varepsilon_{t}-\eta \log \eta\right) \\
\alpha^{\prime} \log \alpha & =\delta_{t}^{\prime}\left(\log \delta_{t}+\mathbf{1} \log z_{t}-\frac{\eta}{1-\eta} \overrightarrow{\mathcal{N}}_{t}{ }_{t}-\frac{1}{1-\eta} \log \varepsilon_{t}-\mathbf{1} \frac{\eta}{1-\eta} \log \eta\right) \\
\alpha^{\prime} \log \alpha & =\log z_{t}-\frac{\eta}{1-\eta} \log \eta+\delta_{t}^{\prime}\left(\log \delta_{t}-\frac{\eta}{1-\eta} \overrightarrow{\mathcal{N}}_{t}{ }_{t}-\frac{1}{1-\eta} \log \varepsilon_{t}\right)
\end{aligned}
$$

Thus, the output in equilibrium is given by:

$$
\log z_{t}=\alpha^{\prime} \log \alpha+\frac{\eta}{1-\eta} \log \eta-\delta_{t}^{\prime}\left(\log \delta_{t}-\frac{\eta}{1-\eta} \overrightarrow{\mathcal{N}}^{\mathcal{S}}{ }_{t}-\frac{1}{1-\eta} \log \varepsilon_{t}\right)
$$

where $\overrightarrow{\mathcal{N}}_{t}{ }_{t}$ is a $n \times 1$ vector with the $i^{\text {th }}$ being $\mathcal{N}_{i, t}^{\mathcal{S}}=\sum_{j} w_{i j, t} \log w_{i j, t}$ and $\delta_{t}$ is also a $n \times 1$ vector with the output shares as in equation (25).

Taking one period difference, we have the equilibrium output growth to be:

$$
\begin{aligned}
\log z_{t+1}-\log z_{t}= & -\left(\delta_{t+1}^{\prime} \log \delta_{t+1}-\delta_{t}^{\prime} \log \delta_{t}\right) \\
& +\frac{\eta}{1-\eta}\left(\delta_{t+1}^{\prime} \overrightarrow{\mathcal{N}^{\mathcal{S}}}{ }_{t+1}-\delta_{t}^{\prime} \overrightarrow{\mathcal{N}}^{\mathcal{S}}{ }_{t}\right) \\
& +\frac{1}{1-\eta}\left(\delta_{t+1}^{\prime} \log \varepsilon_{t+1}-\delta_{t}^{\prime} \log \varepsilon_{t}\right) \\
= & -\left(\mathcal{N}_{t+1}^{\mathcal{C}}-\mathcal{N}_{t}^{\mathcal{C}}\right)+\frac{\eta}{1-\eta}\left(\mathcal{N}_{t+1}^{\mathcal{S}}-\mathcal{N}_{t}^{\mathcal{S}}\right)+\frac{1}{1-\eta}\left(e_{t+1}-e_{t}\right)
\end{aligned}
$$

Furthermore, the equilibrium spot market prices are given by:

$$
\begin{aligned}
\log P_{t} & =\left[\mathrm{I}-\eta W_{t}\right]^{-1}\left[(1-\eta) \log \delta_{t}+(1-\eta) \mathbf{1} \log z_{t}-\eta \overrightarrow{\mathcal{N}}_{t}-\log \varepsilon_{t}-\mathbf{1} \eta \log \eta\right] \\
& =\log z_{t}-\frac{\eta}{1-\eta} \log \eta+(1-\eta)\left[\mathrm{I}-\eta W_{t}\right]^{-1}\left[\log \delta_{t}-\eta \overrightarrow{\mathcal{N}}_{t}{ }_{t}-\log \varepsilon_{t}\right]
\end{aligned}
$$




\section{D.4 Output growth: approximation}

Although we don't have a closed-form solution for $\nu \neq 1$, we can approximated the equilibrium solution around $v=1$. The equilibrium is the solution to system of equations 12 and 15, doing a first order approximation of the solution around $v=1$ yields the following output growth rate:

$$
\begin{aligned}
\log z_{t+1}-\log z_{t} \approx & -\left(\mathcal{N}_{t+1}^{\mathcal{C}}-\mathcal{N}_{t}^{\mathcal{C}}\right)+\frac{\eta}{1-\eta}\left(\mathcal{N}_{t+1}^{\mathcal{S}}-\mathcal{N}_{t}^{\mathcal{S}}\right)+\frac{1}{1-\eta}\left(e_{t+1}-e_{t}\right) \\
& +\frac{\eta}{1-\eta}\left(\delta_{t+1}^{\prime} \Psi_{t+1}-\delta_{t}^{\prime} \Psi_{t}\right)(\nu-1)
\end{aligned}
$$

where $\delta_{t}^{\prime} \Psi_{t}=\sum_{i} \delta_{i, t} \Psi_{i, t}, \delta_{i, t}$ is the output share of firm $i$ when $\nu=1$ and $\Psi_{i, t}$ is given by

$$
\Psi_{i, t} \equiv-\left.\frac{\partial}{\partial \nu} \log \mu_{i, t}\right|_{\nu=1}=\frac{1}{2} \sum_{j} w_{i j, t}\left(\log \frac{w_{i j, t}}{\bar{P}_{j, t}}-\sum_{s} w_{i s, t} \log \frac{w_{i s, t}}{\bar{P}_{s, t}}\right)^{2}>0
$$

where $\bar{P}_{i, t}$ is the market price of good $i$ when $\nu=1$ (equation 26) The detailed derivation of the above expression is in the appendix. The negative sign for the derivative is intuitive: if the firm can substitute input more easily (higher $\nu$ ), then it should be less constrained. Moreover, the slope $\Psi_{i, t}$ is proportional to the input variance using the network weight as probability measure:

$$
\Psi_{i, t}=\frac{1}{2} \sum_{j} w_{i j}\left(\log \bar{y}_{i j, t}-\sum_{s} w_{i s} \log \bar{y}_{i s, t}\right)^{2}=\frac{1}{2} \operatorname{Var}_{i}\left(\log \bar{y}_{i j, t}\right)
$$

where $\bar{y}_{i j, t}$ is the input that firm $i$ would have bought from firm $j$ under $\nu=1$ in equilibrium.

This gives us a clear-cut intuition for this additional factor: it's the average input dispersion under $\nu=1$. If the input dispersion increases on average at the unit elasticity equilibrium, then

$$
\delta_{t+1}^{\prime} \Psi_{t+1}-\delta_{t}^{\prime} \Psi_{t}>0 .
$$

If $\nu>1$, then firms become less constraint (multiplier decreases) as they benefit from input substitution and total output increases. Input dispersion is good (increases output and consumption) when firms substitute inputs more efficiently $(\nu>1)$. 
Derivation Let's combine equations 12 and 15 into one system of $n+1$ equation and $n+1$ unknowns:

$$
\begin{gathered}
f_{i}(x, \nu)=(1-\eta) \log \delta_{i, t}+(1-\eta) \log z_{t}+\eta \log \mu_{i, t}-\log P_{i, t}-\log \varepsilon_{i, t}-\eta \log \eta=0 \quad \forall i=1, \ldots, n \\
f_{n+1}(x, \nu)=\sum_{i} \alpha_{i} \log P_{i, t}-\sum_{i} \alpha_{i} \log \alpha_{i, t}=0
\end{gathered}
$$

where $x=\left(\log P_{1, t}, \ldots, \log P_{n, t}, \log z_{t}\right)$. The function $f: \mathbb{R}^{n+2} \rightarrow \mathbb{R}^{n+1}$ specifies the system of equations that fully characterize the equilibrium. Let $x^{*}(\nu)$ be the solution to

$$
f\left(x^{*}, \nu\right)=0 .
$$

Thus, the equilibrium $x^{*}(\nu)$ depends implicitly on $\nu$. Furthermore, we know the solution when $\nu=1$ from the cobb-douglas case derived in the previous section, and we can approximate the equilibrium $x^{*}(\nu)$ around $\nu=1$ :

$$
\left.\log z_{t} \approx \log z_{t}\right|_{\nu=1}+\left.\frac{\partial}{\partial \nu} \log z_{t}\right|_{\nu=1}(\nu-1)
$$

where the derivative term can be computed using the implicit function theorem to system (27).

The term $\left.\frac{\partial}{\partial \nu} \log z_{t}\right|_{\nu=1}$ is the last entry of

$$
\frac{\partial}{\partial \nu} x_{t}^{*}=-\left[\frac{\partial}{\partial x} f(x, \nu)\right]^{-1} \frac{\partial}{\partial \nu} f(x, \nu)
$$

where

$$
\begin{aligned}
\frac{\partial}{\partial x} f(x, \nu) & =\left[\begin{array}{ccc}
\frac{\partial}{\partial x_{1}} f_{1}(x, \nu) & \cdots & \frac{\partial}{\partial x_{n+1}} f_{1}(x, \nu) \\
\vdots & \ddots & \vdots \\
\frac{\partial}{\partial x_{1}} f_{n+1}(x, \nu) & \cdots & \frac{\partial}{\partial x_{n+1}} f_{n+1}(x, \nu)
\end{array}\right]_{n+1 \times n+1} \\
& =\left[\begin{array}{cccc}
\frac{\partial}{\partial \log P_{1, t}} f_{1} & \cdots & \frac{\partial}{\partial \log P_{n, t}} f_{1} & \frac{\partial}{\partial \log z_{t}} f_{1} \\
\vdots & \ddots & \vdots & \vdots \\
\frac{\partial}{\partial \log P_{1, t}} f_{n+1} & \cdots & \frac{\partial}{\partial \log P_{n, t}} f_{n+1} & \frac{\partial}{\partial \log z_{t}} f_{n+1}
\end{array}\right]_{n+1 \times n+1}
\end{aligned}
$$


and

$$
\frac{\partial}{\partial \nu} f(x, \nu)=\left[\begin{array}{c}
\frac{\partial}{\partial \nu} f_{1}(x, \nu) \\
\vdots \\
\frac{\partial}{\partial \nu} f_{n+1}(x, \nu)
\end{array}\right]_{n+1 \times 1}
$$

where all derivatives are evaluated at $\nu=1$.

Next, we have to calculate of entry of the matrices $\frac{\partial}{\partial x} f(x, \nu)$ and $\frac{\partial}{\partial \nu} f(x, \nu)$. Let's start by computing the entries of $\frac{\partial}{\partial x} f(x, \nu)$ and its inverse:

$$
\begin{array}{rlrl}
\left.\frac{\partial}{\partial \log P_{j, t}} f_{i}\right|_{\nu=1} & =\eta w_{i j, t} \quad & \text { for } i, j=1, \ldots, n \text { and } i \neq j \\
\left.\frac{\partial}{\partial \log P_{i, t}} f_{i}\right|_{\nu=1} & =\eta w_{i j, t}-1 & & \text { for } i=1, \ldots, n \\
\left.\frac{\partial}{\partial \log z_{t}} f_{i}\right|_{\nu=1} & =1-\eta & & \text { for } i=1, \ldots, n \\
\left.\frac{\partial}{\partial \log P_{i, t}} f_{n+1}\right|_{\nu=1} & =\alpha_{i} & & \text { for } i=1, \ldots, n \\
\left.\frac{\partial}{\partial \log z_{t}} f_{n+1}\right|_{\nu=1} & =0 & &
\end{array}
$$

Thus,

$$
\frac{\partial}{\partial x} f(x, y)=\left[\begin{array}{ccccc}
\eta w_{11, t}-1 & \eta w_{12, t} & \ldots & \eta w_{1 n, t} & 1-\eta \\
\eta w_{21, t} & \eta w_{22, t}-1 & \ldots & \eta w_{2 n, t} & 1-\eta \\
\vdots & & \ddots & \vdots & \vdots \\
\eta w_{n 1, t} & \eta w_{n 2, t} & \ldots & \eta w_{n n, t}-1 & 1-\eta \\
\alpha_{1} & \alpha_{2} & \ldots & \alpha_{n} & 0
\end{array}\right]_{n+1 \times n+1}=\left[\begin{array}{cc}
-(\mathbf{I}-\eta W) & \mathbf{1}(1-\eta) \\
\alpha^{\prime} & 0
\end{array}\right]
$$

and its inverse is given by

$$
\left[\frac{\partial}{\partial x} f(x, y)\right]^{-1}=\left[\begin{array}{cc}
-(\mathbf{I}-\eta W) & \mathbf{1}(1-\eta) \\
\alpha & 0
\end{array}\right]^{-1}=\left[\begin{array}{cc}
-\left(\mathrm{I}-\mathbf{1} \alpha^{\prime}\right)(\mathbf{I}-\eta W)^{-1} & \mathbf{1} \\
\alpha^{\prime}(\mathbf{I}-\eta W)^{-1} & 1
\end{array}\right]
$$

Next, let's compute the entries of $\frac{\partial}{\partial \nu} f(x, \nu)$ :

$$
\begin{aligned}
\left.\frac{\partial}{\partial \nu} f_{i}\right|_{\nu=1} & =\left.(1-\eta) \frac{\partial}{\partial \nu} \log \delta_{i, t}\right|_{\nu=1}+\left.\eta \frac{\partial}{\partial \nu} \log \mu_{i, t}\right|_{\nu=1} \quad \text { for } i=1, \ldots, n \\
\left.\frac{\partial}{\partial \nu} f_{n+1}\right|_{\nu=1} & =0
\end{aligned}
$$


where

$$
\begin{aligned}
\left.\frac{\partial}{\partial \nu} \log \mu_{i, t}\right|_{\nu=1} & =\left.\frac{\partial}{\partial \nu} \frac{\log \left(\sum_{j} w_{i j, t}^{\nu} P_{j, t}^{1-\nu}\right)}{1-\nu}\right|_{\nu=1} \\
& =\lim _{\nu \rightarrow 1} \frac{\partial}{\partial \nu} \frac{\log \left(\sum_{j} w_{i j, t}^{\nu} P_{j, t}^{1-\nu}\right)}{1-\nu} \\
& =\lim _{\nu \rightarrow 1} \frac{1}{(1-\nu)^{2}}\left[\frac{(1-\nu)\left(\sum_{j} w_{i j, t}^{\nu} P_{j, t}^{1-\nu}\left(\log w_{i j, t}-\log P_{j, t}\right)\right)}{\sum_{j} w_{i j, t}^{\nu} P_{j, t}^{1-\nu}}+\log \left(\sum_{j} w_{i j, t}^{\nu} P_{j, t}^{1-\nu}\right)\right] \\
& =\lim _{\nu \rightarrow 1} \frac{\sum_{j}(1-\nu) \tilde{w}_{i j, t} \log \frac{w_{i j, t}}{P_{j, t}}+\log \left(\sum_{j} w_{i j, t}^{\nu} P_{j, t}^{1-\nu}\right)}{(1-\nu)^{2}}
\end{aligned}
$$

where $\tilde{w}_{i j, t}=\frac{w_{i j, t}^{\nu} P_{j, t}^{1-\nu}}{\sum_{j} w_{i j, t}^{\nu} P_{j, t}^{1-\nu}}$. Using L'Hôspital's rule twice:

$$
\begin{aligned}
\left.\frac{\partial}{\partial \nu} \log \mu_{i, t}\right|_{\nu=1} & =\lim _{\nu \rightarrow 1} \frac{\sum_{j}(1-\nu) \tilde{w}_{i j, t} \log \frac{w_{i j, t}}{P_{j, t}}+\log \left(\sum_{j} w_{i j, t}^{\nu} P_{j, t}^{1-\nu}\right)}{(1-\nu)^{2}} \\
& =\lim _{\nu \rightarrow 1} \frac{\sum_{j}\left((1-\nu) \frac{\partial \tilde{w}_{i j, t}}{\partial \nu}-\tilde{w}_{i j, t}\right) \log \frac{w_{i j, t}}{P_{j, t}}+\frac{\sum_{j} w_{i j, t}^{\nu} t_{j, t}^{1-\nu} \log \frac{w_{i j, t}}{P_{j, t}}}{\sum_{j} w_{i j, t}^{\nu} P_{j, t}^{1-\nu}}}{-2(1-\nu)} \\
& =\lim _{\nu \rightarrow 1} \frac{\sum_{j}\left((1-\nu) \frac{\partial \tilde{w}_{i j, t}}{\partial \nu}-\tilde{w}_{i j, t}\right) \log \frac{w_{i j, t}}{P_{j, t}}+\sum_{j} \tilde{w}_{i j, t} \log \frac{w_{i j, t}}{P_{j, t}}}{-2(1-\nu)} \\
& =\lim _{\nu \rightarrow 1} \frac{\sum_{j}\left((1-\nu) \frac{\partial^{2} \tilde{w}_{i j, t}}{\partial \nu^{2}}-2 \frac{\partial \tilde{w}_{i j, t}}{\partial \nu}\right) \log \frac{w_{i j, t}}{P_{j, t}}+\sum_{j} \frac{\partial \tilde{w}_{i j, t}}{\partial \nu} \log \frac{w_{i j, t}}{P_{j, t}}}{2} \\
& =\lim _{\nu \rightarrow 1} \frac{\sum_{j}\left((1-\nu) \frac{\partial^{2} \tilde{w}_{i j, t}}{\partial \nu^{2}}-\frac{\partial \tilde{w}_{i j, t}}{\partial \nu}\right) \log \frac{w_{i j, t}}{P_{j, t}}}{2} \\
& =\frac{1}{2} \lim _{\nu \rightarrow 1} \sum_{j}\left((1-\nu) \frac{\partial^{2} \tilde{w}_{i j, t}}{\partial \nu^{2}}-\frac{\partial \tilde{w}_{i j, t}}{\partial \nu}\right) \log \frac{w_{i j, t}}{P_{j, t}} \\
& =-\frac{1}{2} \lim _{\nu \rightarrow 1} \sum_{j}\left(\frac{\partial \tilde{w}_{i j, t}}{\partial \nu}\right) \log \frac{w_{i j, t}}{P_{j, t}} \\
& =-\frac{1}{2} \sum_{j} w_{i j, t}\left(\log \frac{w_{i j, t}}{\bar{P}_{j, t}}-\sum_{s} w_{i s, t} \log \frac{w_{i s, t}}{\bar{P}_{s, t}}\right)^{2} \log \frac{w_{i j, t}}{\bar{P}_{j, t}} \\
& =-\frac{1}{2} \sum_{j} w_{i j, t}\left(\log \frac{w_{i j, t}}{\bar{P}_{j, t}}-\sum_{s} w_{i s, t} \log \frac{w_{i s, t}}{\bar{P}_{s, t}}\right)^{2}=-\Psi_{i, t}
\end{aligned}
$$


The term $\left.\frac{\partial}{\partial \nu} \log \delta_{i, t}\right|_{\nu=1}$ has to be computed the implicit function theorem. $\left\{\delta_{i, t}\right\}_{i}$ is the solution to the following system of equation:

$$
g_{i}\left(\delta_{t}, \nu\right)=0 \quad \forall i=1, \ldots, n
$$

where

$$
g_{i}\left(\delta_{t}, \nu\right)=(1-\eta) \alpha_{i}+\eta \sum_{j} \tilde{w}_{j i, t} \delta_{j, t}-\delta_{i, t}
$$

Therefore,

$$
\begin{aligned}
\frac{\partial}{\partial \nu} \delta_{t} & =-\left[\frac{\partial}{\partial \delta_{t}} g\left(\delta_{t}, \nu\right)\right]^{-1} \frac{\partial}{\partial \nu} g\left(\delta_{t}, \nu\right) \\
& =\left[\mathrm{I}-\eta \tilde{W}^{\prime}\right]^{-1}\left[\begin{array}{c}
\eta \sum_{j} \delta_{j, t} \frac{\partial}{\partial \nu} \tilde{w}_{j 1, t} \\
\vdots \\
\eta \sum_{j} \delta_{j, t} \frac{\partial}{\partial \nu} \tilde{w}_{j n, t}
\end{array}\right]
\end{aligned}
$$

where

$$
\left.\frac{\partial}{\partial \nu} \tilde{w}_{i j, t}\right|_{\nu=1}=w_{i j, t}\left(\log \frac{w_{i j, t}}{\bar{P}_{j, t}}-\sum_{s} w_{i s} \log \frac{w_{i s, t}}{\bar{P}_{s, t}}\right) \equiv w_{i j, t}^{-}
$$

Notice that $\sum_{j} \bar{w}_{i j}=0$ and $\left.\sum_{j} \frac{\partial}{\partial \nu} \delta_{j, t}\right|_{\nu=1}=0$. Let $\bar{W}_{t}$ be a $n \times n$ matrix whose element $(i, j)$ is $\bar{w}_{i j, t}$. Thus,

$$
\begin{aligned}
\left.\frac{\partial}{\partial \nu} \delta_{t}\right|_{\nu=1} & =\left[\mathrm{I}-\eta W_{t}^{\prime}\right]^{-1} \bar{W}_{t}^{\prime} \bar{\delta}_{t} \\
\left.\frac{\partial}{\partial \nu} \log \delta_{i, t}\right|_{\nu=1} & =\left.\frac{1}{\bar{\delta}_{i, t}} \frac{\partial}{\partial \nu} \delta_{i, t}\right|_{\nu=1}
\end{aligned}
$$

and

$$
\frac{\partial}{\partial \nu} f(x, \nu)=\left[\begin{array}{c}
\left.(1-\eta) \frac{1}{\delta_{1, t}} \frac{\partial}{\partial \nu} \delta_{1, t}\right|_{\nu=1}-\eta \Psi_{1, t} \\
\vdots \\
\left.(1-\eta) \frac{1}{\delta_{n, t}} \frac{\partial}{\partial \nu} \delta_{n, t}\right|_{\nu=1}-\eta \Psi_{n, t} \\
0
\end{array}\right]
$$

Altogether yields: 


$$
\begin{aligned}
\frac{\partial}{\partial \nu} x_{t}^{*} & =-\left[\frac{\partial}{\partial x} f(x, \nu)\right]^{-1} \frac{\partial}{\partial \nu} f(x, \nu) \\
& =-\left[\begin{array}{cc}
-\left(\mathbf{I}-\mathbf{1} \alpha^{\prime}\right)(\mathbf{I}-\eta W)^{-1} & \mathbf{1} \\
\alpha^{\prime}(\mathbf{I}-\eta W)^{-1} & 1
\end{array}\right]\left[\begin{array}{c}
\left.(1-\eta) \frac{1}{\delta_{1, t}} \frac{\partial}{\partial \nu} \delta_{1, t}\right|_{\nu=1}-\eta \Psi_{1, t} \\
\vdots \\
\left.(1-\eta) \frac{1}{\delta_{n, t}} \frac{\partial}{\partial \nu} \delta_{n, t}\right|_{\nu=1}-\eta \Psi_{n, t} \\
0
\end{array}\right]
\end{aligned}
$$

and, since $\frac{\partial}{\partial \nu} \log z_{t}$ is the last entry of the vector above,

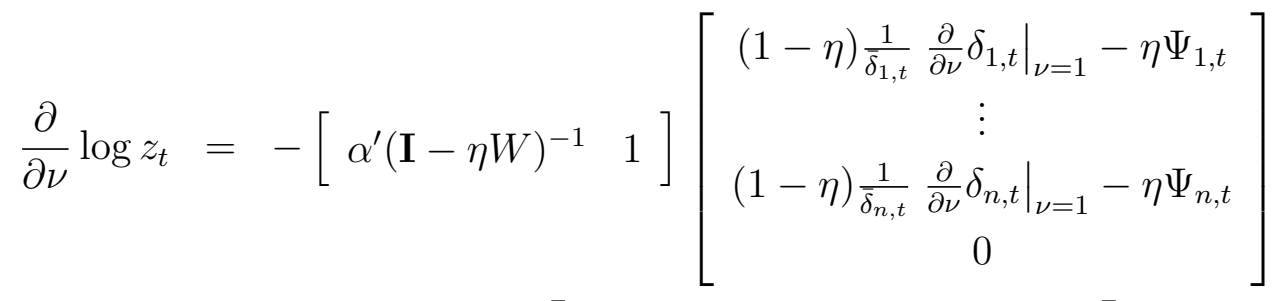

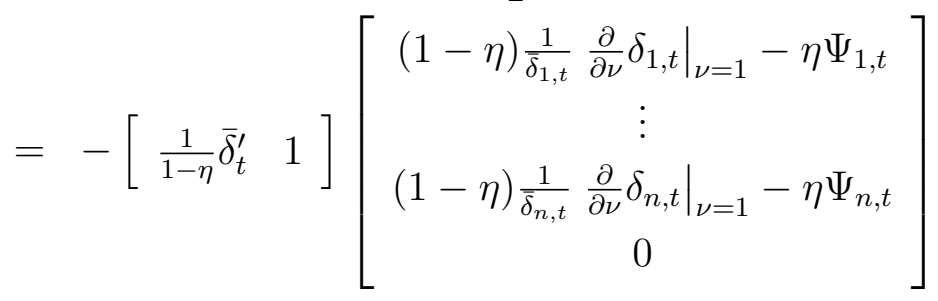

$$
\begin{aligned}
& =-\sum_{j} \frac{1}{1-\eta} \bar{\delta}_{j, t}\left(\left.(1-\eta) \frac{1}{\bar{\delta}_{j, t}} \frac{\partial}{\partial \nu} \delta_{j, t}\right|_{\nu=1}-\eta \Psi_{j, t}\right) \\
& =-\sum_{j} \frac{1}{1-\eta} \bar{\delta}_{j, t}\left(\left.(1-\eta) \frac{1}{\bar{\delta}_{j, t}} \frac{\partial}{\partial \nu} \delta_{j, t}\right|_{\nu=1}-\eta \Psi_{j, t}\right) \\
& =-\left.\sum_{j} \frac{\partial}{\partial \nu} \delta_{j, t}\right|_{\nu=1}+\frac{\eta}{1-\eta} \sum_{j} \bar{\delta}_{j, t} \Psi_{j, t} \\
& =\frac{\eta}{1-\eta} \sum_{j} \bar{\delta}_{j, t} \Psi_{j, t} \\
& =\frac{\eta}{1-\eta} \bar{\delta}_{t}^{\prime} \Psi_{t}
\end{aligned}
$$


Hence, the output can be approximated by

$$
\begin{aligned}
\log z_{t} & \left.\approx \log z_{t}\right|_{\nu=1}+\left.\frac{\partial}{\partial \nu} \log z_{t}\right|_{\nu=1}(\nu-1) \\
& =\left.\log z_{t}\right|_{\nu=1}+\frac{\eta}{1-\eta} \bar{\delta}_{t}^{\prime} \Psi_{t}(\nu-1) \\
& =\alpha^{\prime} \log \alpha+\frac{\eta}{1-\eta} \log \eta-\bar{\delta}_{t}^{\prime}\left(\log \bar{\delta}_{t}-\frac{\eta}{1-\eta} \overrightarrow{\mathcal{N}}_{t}+\frac{1}{1-\eta} \log \varepsilon_{t}\right)+\frac{\eta}{1-\eta} \bar{\delta}_{t}^{\prime} \Psi_{t}(\nu-1)
\end{aligned}
$$

and the approximation for the output growth is given by

$$
\begin{aligned}
\log z_{t+1}-\log z_{t} \approx & -\left(\bar{\delta}_{t+1}^{\prime} \log \bar{\delta}_{t+1}-\bar{\delta}_{t}^{\prime} \log \bar{\delta}_{t}\right)+\frac{\eta}{1-\eta}\left(\bar{\delta}_{t+1}^{\prime} \overrightarrow{\mathcal{N}}_{t+1}^{\mathcal{S}}-\bar{\delta}_{t}^{\prime} \overrightarrow{\mathcal{N}}_{t}^{\mathcal{S}}\right) \\
& +\frac{1}{1-\eta}\left(\bar{\delta}_{t+1}^{\prime} \log \varepsilon_{t+1}-\bar{\delta}_{t}^{\prime} \log \varepsilon_{t}\right)+\frac{\eta}{1-\eta}\left(\bar{\delta}_{t+1}^{\prime} \Psi_{t+1}-\bar{\delta}_{t}^{\prime} \Psi_{t}\right)(\nu-1) \\
= & -\left(\mathcal{N}_{t+1}^{\mathcal{C}}-\mathcal{N}_{t}^{\mathcal{C}}\right)+\frac{\eta}{1-\eta}\left(\mathcal{N}_{t+1}^{\mathcal{S}}-\mathcal{N}_{t}^{\mathcal{S}}\right)+\frac{1}{1-\eta}\left(e_{t+1}-e_{t}\right) \\
& +\frac{\eta}{1-\eta}\left(\bar{\delta}_{t+1}^{\prime} \Psi_{t+1}-\bar{\delta}_{t}^{\prime} \Psi_{t}\right)(\nu-1)
\end{aligned}
$$

\section{E Stochastic Discount Factor Derivation}

In this section, I'll do the detailed derivation of the wealth consumption expenditure ratio,

the stochastic discount factor and the prices of risk. The unlevered consumption claim is fully described by

$$
\begin{aligned}
\log z_{t+1}-\log z_{t} & =\phi_{\mathcal{N}^{c}} \Delta \mathcal{N}_{t+1}^{\mathcal{C}}+\phi_{\mathcal{N}^{\mathcal{S}}} \Delta \mathcal{N}_{t+1}^{\mathcal{S}}+\phi_{e} \Delta e_{t+1}+x_{t} \\
\Delta \mathcal{N}_{t+1}^{\mathcal{C}} & =\sigma_{\mathcal{N}^{c}} \varepsilon_{\mathcal{N}^{c}, t+1} \\
\Delta \mathcal{N}_{t+1}^{\mathcal{S}} & =\sigma_{\mathcal{N}^{\mathcal{S}} \varepsilon_{\mathcal{N}^{\mathcal{S}}, t+1}} \\
\Delta e_{t+1} & =\sigma_{e} \varepsilon_{e, t+1} \\
x_{t+1} & =\rho_{x} x_{t}+\sigma_{x} \varepsilon_{x, t+1}
\end{aligned}
$$

where $\varepsilon_{e, t+1}, \varepsilon_{\mathcal{N}^{c}, t+1}, \varepsilon_{\mathcal{N}^{\mathcal{S}}, t+1}$ and $\varepsilon_{x, t+1}$ are i.i.d. standard normal random variables.

The return on total wealth can be approximated (1st order approximation) by

$$
r_{t+1}^{W}=\kappa_{0}^{c}+\Delta \omega_{t+1}+w c_{t+1}-\kappa_{1}^{c} w c_{t}
$$


where

$$
\begin{aligned}
\kappa_{0}^{c} & =\log \left(\exp \left(\mu_{w c}\right)-1\right)+\frac{\exp \left(\mu_{w c}\right)}{\exp \left(\mu_{w c}\right)-1} \mu_{w c} \\
\kappa_{1}^{c} & =\frac{\exp \left(\mu_{w c}\right)}{\exp \left(\mu_{w c}\right)-1}>1
\end{aligned}
$$

and $\mu_{w c}$ is the unconditional average of the wealth consumption expenditure ratio.

I'll start from the guess that the wealth-consumption expenditure ratio is a linear on $x_{t}$

$$
w c_{t}=\mu_{w c}+A x_{t}
$$

where $A$ is a constant. Substituting the wealth-consumption ratio expression in the return approximation yields:

$$
r_{t+1}^{W}=r_{0}^{c}+\beta_{x} x_{t}+\beta_{\varepsilon e} \sigma_{e} \varepsilon_{e, t+1}+\beta_{\varepsilon x} \sigma_{x} \varepsilon_{x, t+1}+\beta_{\varepsilon \mathcal{N}^{c}} \sigma_{\mathcal{N}^{c}} \varepsilon_{\mathcal{N}^{c}, t+1}+\beta_{\varepsilon \mathcal{N}^{\mathcal{S}}} \sigma_{\mathcal{N}^{\mathcal{S}} \varepsilon_{\mathcal{N}^{\mathcal{S}}, t+1}}
$$

where

$$
\begin{aligned}
r_{0}^{c} & =\kappa_{0}^{c}-\mu_{w c}\left(\kappa_{1}^{c}-1\right) \\
\beta_{x} & =\phi_{x} \phi_{e}-A\left(\kappa_{1}^{c}-\rho_{x}\right) \\
\beta_{\varepsilon e} & =\phi_{e} \\
\beta_{\varepsilon x} & =A \\
\beta_{\varepsilon \mathcal{N}^{c}} & =\phi_{\mathcal{N}^{c}} \\
\beta_{\varepsilon \mathcal{N}^{\mathcal{S}}} & =\phi_{\mathcal{N}^{\mathcal{S}}}
\end{aligned}
$$

Using lemma 1, the stochastic discount factor is given by

$$
\begin{aligned}
m_{t+1} & =\theta \log \beta-\rho \theta \Delta z_{t+1}+(\theta-1) r_{t+1}^{\mathcal{W}} \\
& =\mu_{s}+\lambda_{x} x_{t}-\lambda_{\varepsilon e} \sigma_{e} \varepsilon_{e, t+1}-\lambda_{\varepsilon x} \sigma_{x} \varepsilon_{x, t+1}-\lambda_{\varepsilon \mathcal{N}^{c}} \sigma_{\mathcal{N}^{c}} \varepsilon_{\mathcal{N}^{\mathcal{C}}, t+1}-\lambda_{\varepsilon \mathcal{N}^{\mathcal{S}}} \sigma_{\mathcal{N}^{\mathcal{S}}} \varepsilon_{\mathcal{N}^{\mathcal{S}}, t+1}
\end{aligned}
$$


where

$$
\begin{aligned}
\mu_{s} & =\theta \log \beta+(\theta-1)\left(\kappa_{0}^{c}-\mu_{w c}\left(\kappa_{1}^{c}-1\right)\right) \\
\lambda_{x} & =-\gamma \phi_{e} \phi_{x}-(\theta-1) A\left(\kappa_{1}^{c}-\rho_{x}\right) \\
\lambda_{\varepsilon e} & =\gamma \phi_{e} \\
\lambda_{\varepsilon x} & =-(\theta-1) A \\
\lambda_{\varepsilon \mathcal{N}^{c}} & =\gamma \phi_{\mathcal{N}^{c}} \\
\lambda_{\varepsilon \mathcal{N}^{\mathcal{S}}} & =\gamma \phi_{\mathcal{N}^{\mathcal{S}}}
\end{aligned}
$$

The euler equation for the return on total wealth is given by

$$
\begin{aligned}
0= & \mu_{s}+r_{0}^{c}+\left(\beta_{x}+\lambda_{x}\right) x_{t} \\
& +\frac{1}{2}\left[\left(\beta_{\varepsilon x}-\lambda_{\varepsilon x}\right)^{2} \sigma_{e}^{2}+\left(\beta_{\varepsilon e}-\lambda_{\varepsilon e}\right)^{2} \sigma_{e}^{2}+\left(\beta_{\varepsilon \mathcal{N}^{c}}-\lambda_{\varepsilon \mathcal{N}^{\mathcal{C}}}\right)^{2} \sigma_{\mathcal{N}^{\mathcal{c}}}^{2}+\left(\beta_{\varepsilon \mathcal{N}^{\mathcal{S}}}-\lambda_{\varepsilon \mathcal{N}^{\mathcal{S}}}\right)^{2} \sigma_{\mathcal{N}^{\mathcal{S}}}^{2}\right]
\end{aligned}
$$

Using method of undetermined coefficients, $A$ solves

$$
\begin{aligned}
0= & \beta_{x}+\lambda_{x} \\
= & \phi_{e} \phi_{x}-A\left(\kappa_{1}^{c}-\rho_{x}\right)-\gamma \phi_{e} \phi_{x}-(\theta-1) A\left(\kappa_{1}^{c}-\rho_{x}\right) \\
= & -\phi_{e} \phi_{x}(\gamma-1)-A \theta\left(\kappa_{1}^{c}-\rho_{x}\right) \\
& \therefore A=-\frac{\phi_{e} \phi_{x}(\gamma-1)}{\theta\left(\kappa_{1}^{c}-\rho_{e}\right)}=(1-\rho) \frac{\phi_{e} \phi_{x}}{\kappa_{1}^{c}-\rho_{x}}
\end{aligned}
$$

and $\mu_{w c}$ solves the following nonlinear equation:

$0=\mu_{s}+r_{0}^{c}+\frac{1}{2}\left[\left(\beta_{\varepsilon e}-\lambda_{\varepsilon e}\right)^{2} \sigma_{e}^{2}+\left(\beta_{\varepsilon x}-\lambda_{\varepsilon x}\right)^{2} \sigma_{x}^{2}+\left(\beta_{\varepsilon \mathcal{N}^{\mathcal{C}}}-\lambda_{\varepsilon \mathcal{N}^{\mathcal{C}}}\right)^{2} \sigma_{\mathcal{N}^{\mathcal{C}}}^{2}+\left(\beta_{\varepsilon \mathcal{N}^{\mathcal{S}}}-\lambda_{\varepsilon \mathcal{N}^{\mathcal{S}}}\right)^{2} \sigma_{\mathcal{N}^{\mathcal{S}}}^{2}\right]$

\section{E.1 Portfolio}

Let the portfolio $i$ dividend growth process be described by

$\Delta d_{i, t+1}=\mu_{i}+\phi_{x}^{i} x_{t}+\varphi_{e}^{i} \sigma_{e} \varepsilon_{e, t+1}+\varphi_{x}^{i} \sigma_{x} \varepsilon_{x, t+1}+\varphi_{\mathcal{N}^{\mathcal{c}}}^{i} \sigma_{\mathcal{N}^{\mathcal{c}}} \varepsilon_{\mathcal{N}^{\mathcal{c}}, t+1}+\varphi_{\mathcal{N}^{\mathcal{S}}}^{i} \sigma_{\mathcal{N}^{\mathcal{S}} \varepsilon_{\mathcal{N}^{\mathcal{S}}, t+1}+\zeta^{i} \sigma_{i} \varepsilon_{i, t+1}}$

We guess and verify that the log price-dividend ratio is linear on $x_{t}$ :

$$
p d_{i, t}=\mu_{i, p d}+A_{i} x_{t}
$$


As the wealth return, the stock returns are approximated by:

$$
r_{i, t+1}=\Delta d_{i, t+1}+\kappa_{0}^{i}+\kappa_{1}^{i} p d_{i, t+1}-p d_{i, t}
$$

where $\kappa_{1}^{i}=\frac{\exp \left(\mu_{i, p d}\right)}{1+\exp \left(\mu_{i, p d}\right)}$ and $\kappa_{0}^{i}=\log \left(1+\exp \left(\mu_{i, p d}\right)\right)-\kappa_{1}^{i} \mu_{i, p d}$ are the approximation constants. Substituting the price-dividend expression into the return approximation yields

$$
\begin{aligned}
& r_{i, t+1}=\Delta d_{i, t+1}+\kappa_{0}^{i}+\kappa_{1}^{i} p d_{i, t+1}-p d_{i, t}
\end{aligned}
$$

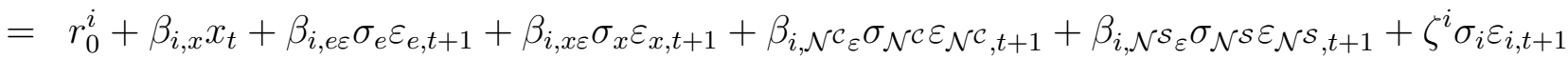

where

$$
\begin{aligned}
r_{0}^{i} & =\mu_{i}+\mu_{i, p d}\left(\kappa_{1}^{i}-1\right)+\kappa_{0}^{i} \\
\beta_{i, x} & =\phi_{x}^{i}-A_{i}\left(1-\kappa_{1}^{i} \rho_{x}\right) \\
\beta_{i, e \varepsilon} & =\varphi_{e}^{i} \\
\beta_{i, x \varepsilon} & =\varphi_{x}^{i}+\kappa_{1}^{i} A_{i} \\
\beta_{i, \mathcal{N}^{\mathcal{C}} \varepsilon} & =\varphi_{\mathcal{N}^{\mathcal{C}}}^{i} \\
\beta_{i, \mathcal{N}^{\mathcal{S}} \varepsilon} & =\varphi_{\mathcal{N}^{\mathcal{S}}}^{i}
\end{aligned}
$$

The euler equation for portfolios $i$ is given by:

$$
\begin{aligned}
0= & \mu_{s}+r_{i}^{c}+\left(\beta_{i, x}+\lambda_{x}\right) x_{t} \\
& +\frac{1}{2}\left[\left(\beta_{i, e \varepsilon}-\lambda_{\varepsilon e}\right)^{2} \sigma_{e}^{2}+\left(\beta_{i, x \varepsilon}-\lambda_{\varepsilon x}\right)^{2} \sigma_{x}^{2}+\left(\beta_{i, \mathcal{N}^{\mathcal{C}} \varepsilon}-\lambda_{\varepsilon \mathcal{N}^{\mathcal{C}}}\right)^{2} \sigma_{\mathcal{N}^{\mathcal{C}}}^{2}+\left(\beta_{i, \mathcal{N}^{\mathcal{S}} \varepsilon}-\lambda_{\varepsilon \mathcal{N}^{\mathcal{S}}}\right)^{2} \sigma_{\mathcal{N}^{\mathcal{S}}}^{2}+\zeta^{i^{2}} \sigma_{i}^{2}\right]
\end{aligned}
$$

Using method of undetermined coefficients, $A_{i}$ solves

$$
\begin{aligned}
0 & =\beta_{i, x}+\lambda_{x} \\
0 & =\phi_{x}^{i}-A_{i}\left(1-\kappa_{1}^{i} \rho_{x}\right)+\lambda_{x} \\
A_{i} & =\frac{\phi_{x}^{i}+\lambda_{x}}{1-\kappa_{1}^{i} \rho_{x}}
\end{aligned}
$$

and $\mu_{i, p d}$ solves the following nonlinear equation

$$
\begin{aligned}
0= & \mu_{s}+r_{i}^{c} \\
& +\frac{1}{2}\left[\left(\beta_{i, e \varepsilon}-\lambda_{\varepsilon e}\right)^{2} \sigma_{e}^{2}+\left(\beta_{i, x \varepsilon}-\lambda_{\varepsilon x}\right)^{2} \sigma_{x}^{2}+\left(\beta_{i, \mathcal{N}^{\mathcal{C}} \varepsilon}-\lambda_{\varepsilon \mathcal{N} \mathcal{c}}\right)^{2} \sigma_{\mathcal{N}^{\mathcal{C}}}^{2}+\left(\beta_{i, \mathcal{N}^{\mathcal{S}} \varepsilon}-\lambda_{\varepsilon \mathcal{N}^{\mathcal{S}}}\right)^{2} \sigma_{\mathcal{N}^{\mathcal{S}}}^{2}+\zeta^{i^{2}} \sigma_{i}^{2}\right]
\end{aligned}
$$




\section{F Data Construction}

\section{F.1 Network data}

The input-output network matrix is necessary to compute the asset pricing factors discussed in this paper. The main input-output data source are the BEA Input-Output tables, however this data set is only available from 1997 to 2012 on annual basis. Due to the short BEA sample, I compute an estimate of the IO table based on the Compustat segment customer data, which is on annual basis from 1979 to 2013. If a customer represent more than $10 \%$ of the seller's sales, then the customer's name is reported in the compustat customer segment data as well as the sales to that particular customer. Cohen and Frazzini (2008) located the CRSP permanent number, PERMNO, of the customer until the year of 2009 and I updated their data set by locating the customer identification number up to 2013. Therefore, from this data set, it's possible to get the some entries of the network matrix $W$, however the resulting network is truncated since all supplier-customers transaction are not observed. The model is at the sector level, so in order to make data and model compatible, compustat customer sales data is aggregate by firm's naics code at the 2-digit sector level.

In order to mitigate the truncation issue, I consider three alternatives ways to compute the the network matrix: (i) assume that all non observed entries are equal to zero and normalize each row to sum one, (ii) equally distributing the reaming weight across sectors, or (iii) assume that all non observed entries are equal to zero and compute the factors based on the truncated network. Besides the network calculation itself, we can compute the output shares in two distinct ways as well: (i) use the compustat reported total sales, or (ii) use only sales reported in the compustat customer segment data in order respect the network truncation. This will result in two distinct ways to compute the shares $\delta$. Hence, for each of $\delta$ type, we may compute the concentration factor directly from the $\delta$, and the sparsity factor may be computed for each of the three alternative networks considered.

To choose which network to use, I compare each one of them to factor copmuted from the the BEA input-output tables. The BEA data is from 1997 to 2012, so I chose the factors calculation based on their correlations with the BEA factors. Figures 7 plots all the factors and their respective innovations using the $\delta$ based on the compustat customer segment data and Figure 8 plots the factors based on the compustat total sales. The calculations that result in the highest correlation with the BEA data is using compustat customer segment sales and equally distributing the remaining network weights across sectors (solid blue line in both Figures).

Figure ?? shows the average fraction of sales to a particular customer type: Foreign Gov-

ernments, US Government (Federal, State and Local), Foreign Countries (not US market), 
Figure 7: BEA amd Compustat Network Factors: using total customer segment sales
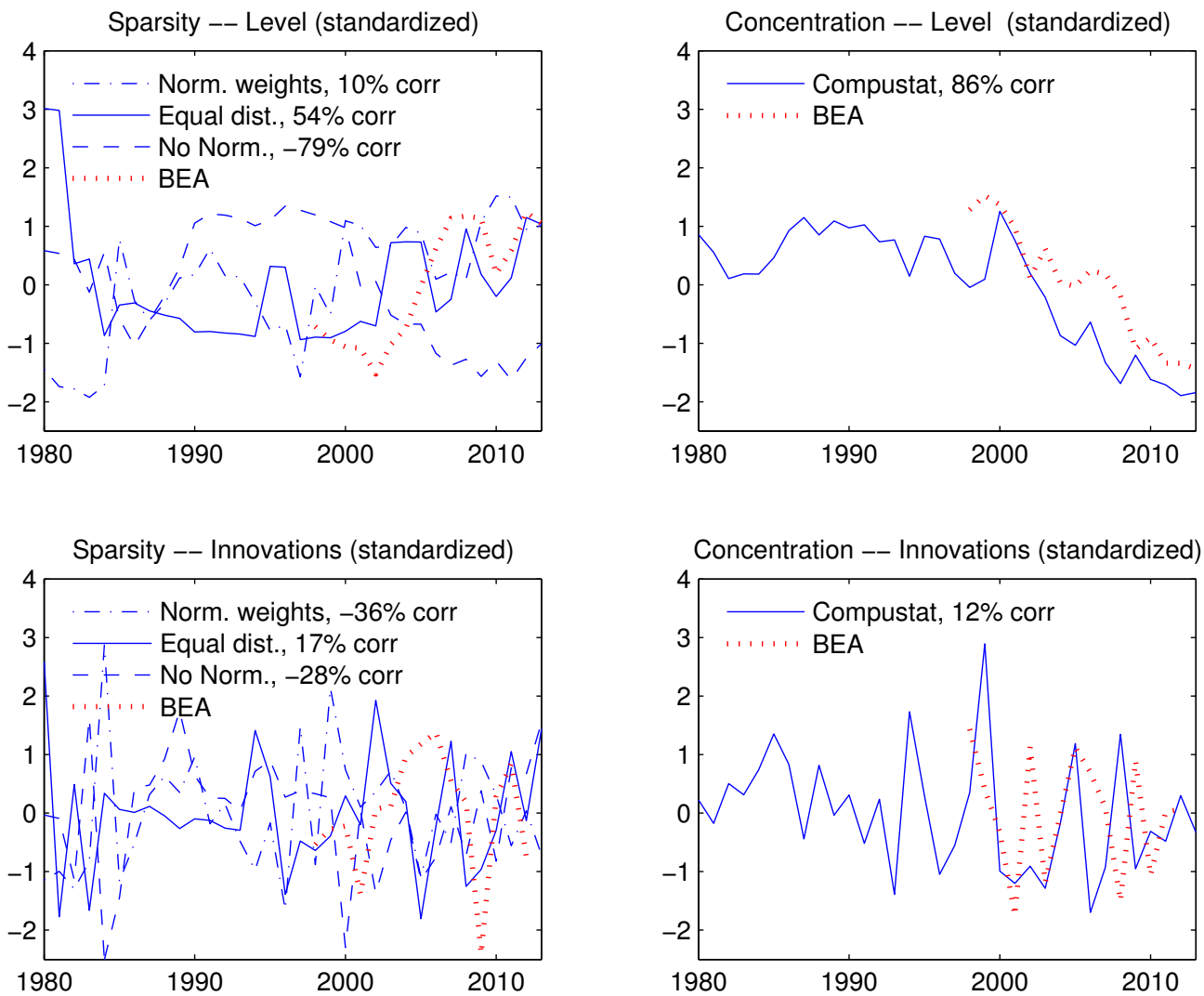

other Companies and companies that had their CRSP identification located. The fraction of customers with CRSP PERMNO identified are roughly constant over time.

The output shares also depend both on the network and on the preference weights. The BEA input-output table report the consumption of sector by the final consumer. Using the consumption of the final consumer to compute the preference weights, we may compare the concentration factor implied by the model (i.e. using the network and preference weights) with and without keeping the preference weights constant. Figure 9 reports the concentration factor using the BEA output shares directly (solid blue line), using model implied shares keeping preferences weights constant (avg. consumption expenditure shares), and using model implied shares with consumption expenditure shares $(\alpha)$ varying over time as well. The resulting time series suggests that the concentration is almost entirely driven by changes in the network rather than changes in the household preferences. 
Figure 8: BEA amd Compustat Network Factor: using total sales
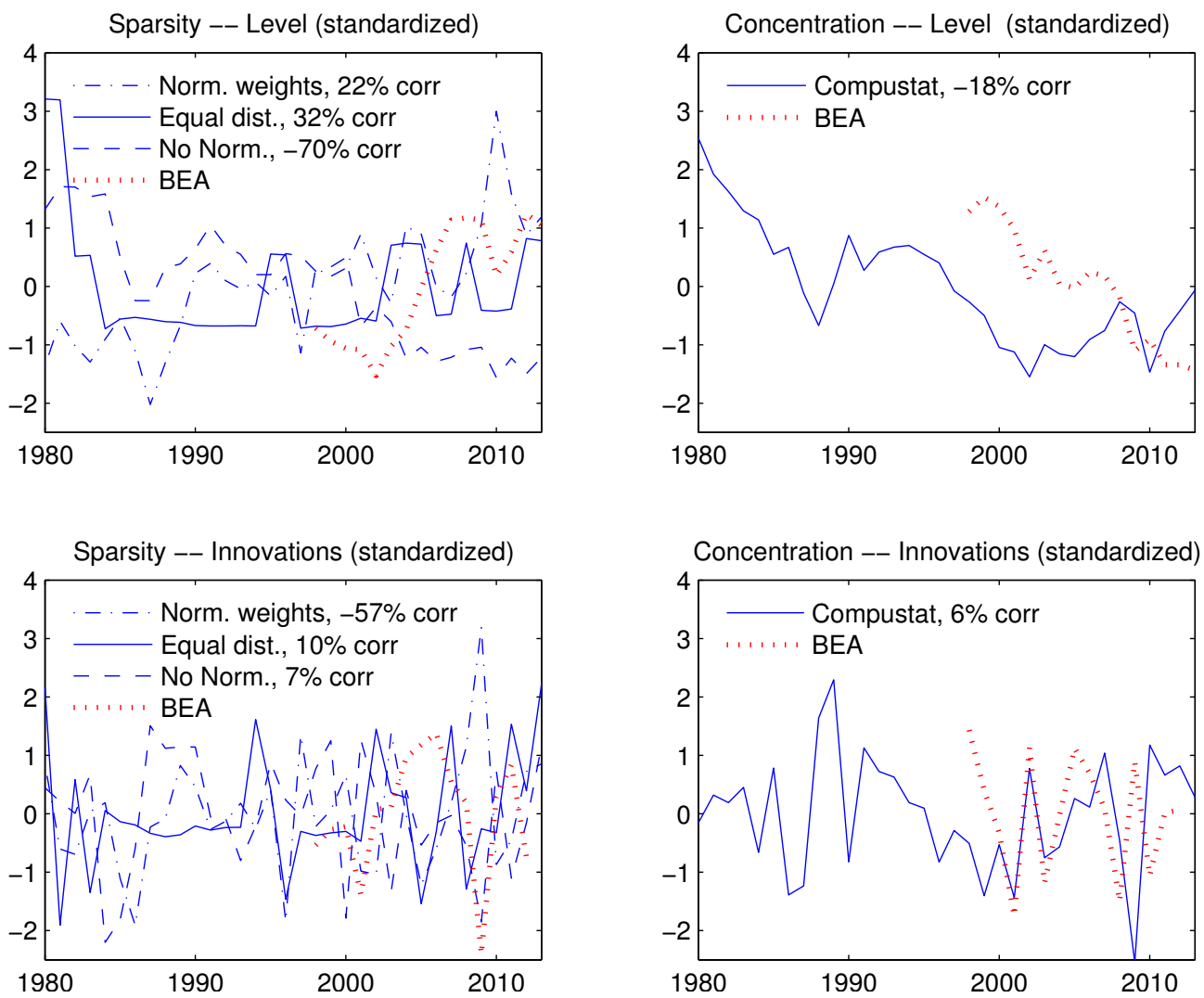
Figure 9: Concentration Factors: using model implied shares and directly using shares from BEA

This Figure reports the concentration factor using the BEA output shares directly (solid blue line), using model implied shares keeping alpha constant (avg. consumption expenditure shares), and using model implied shares with consumption expenditure shares (alphas) varying over time as well. All series are standardized.

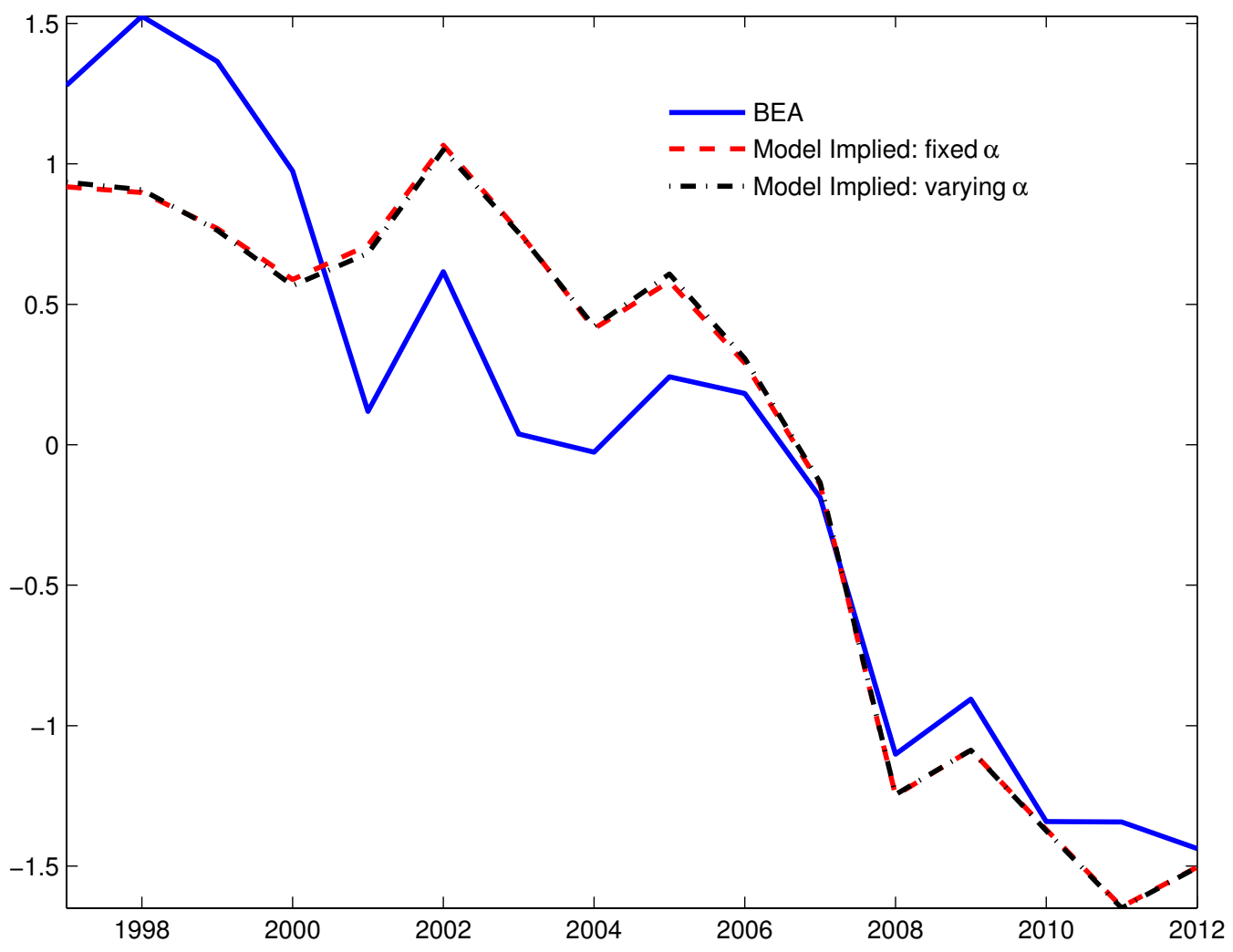


Table 6: Double Sort

This Table reports average excess return in panel A, post sample CAPM alphas in panel B and post sample Fama French alphas in panel C. Stocks are independently double sorted on sparsity-beta and concentration-beta, and double-sorted portfolios are formed by terciles.

\begin{tabular}{lccccc}
\hline \multicolumn{5}{c}{ Panel A: returns } \\
Concentration \\
Sparsity & $(1)$ & $(2)$ & $(3)$ & $(3)-(1)$ & t-stat \\
$(1)$ & 10.38 & 7.95 & 4.56 & -5.82 & -1.58 \\
$(2)$ & 12.79 & 7.56 & 8.54 & -4.25 & -1.29 \\
$(3)$ & 10.95 & 10.71 & 15.82 & 4.87 & 1.27 \\
$(3)-(1)$ & 0.57 & 2.76 & 11.26 & - & - \\
t-stat & 0.13 & 0.72 & 3.00 & - & - \\
\hline \multicolumn{5}{c}{ Panel B: $\alpha_{C A P M}$} \\
Sparsity & $(1)$ & $(2)$ & $(3)$ & $(3)-(1)$ & t-stat \\
$(1)$ & 1.18 & 0.07 & -4.06 & -5.24 & -1.41 \\
$(2)$ & 4.62 & 1.74 & 2.40 & -2.22 & -0.69 \\
$(3)$ & 4.11 & 4.72 & 8.73 & 4.61 & 1.18 \\
$(3)-(1)$ & 2.93 & 4.66 & 12.79 & - & - \\
t-stat & 0.68 & 1.23 & 3.42 & - & - \\
\hline \multicolumn{7}{c}{ Panel C: $\alpha_{F F}$} \\
Sparsity & $(1)$ & $(2)$ & $(3)$ & $(3)-(1)$ & t-stat \\
(1) & 0.47 & -0.10 & -4.56 & -5.03 & -1.40 \\
$(2)$ & 5.00 & 0.82 & 0.84 & -4.16 & -1.42 \\
(3) & 2.99 & 3.52 & 8.87 & 5.88 & 1.63 \\
(3)-(1) & 2.52 & 3.63 & 13.43 & - & - \\
t-stat & 0.63 & 0.99 & 3.68 & - & - \\
\hline \hline
\end{tabular}




\section{Table 7: Robustness}

This Table reports average excess return in panel A, post sample CAPM alphas in panel B and post sample Fama French alphas in panel C. Stocks are sorted on sparsity-beta and concentration-beta, and one-way sorted portfolios are formed by terciles. Each row corresponds to a different specification: (1) is benhmark estimation, (2) excludes network factors in level from the exposure estimation in regression 18. (3) considers all CRSP stocks, i.e. stocks with and without network data, (4) considers only CRSP stocks without network data, (5) uses innovations in residual TFP as consumption growth orthogonalized to network factors' innovations, (6) excludes residual TFP from beta estimations, (7) uses equation 16 to directly compute the residual TFP, but using aggregate consumption growth rather than TFP growth, and (8) uses equation 16 to directly compute the residual TFP using reasonable returns to scale $\eta=.35$, (9) uses 16 years as trailing window, (10) uses 17 years as trailing window, (11) uses 18 years as trailing window, (12) uses 19 years as trailing window, and (13) uses 20 years as trailing window.

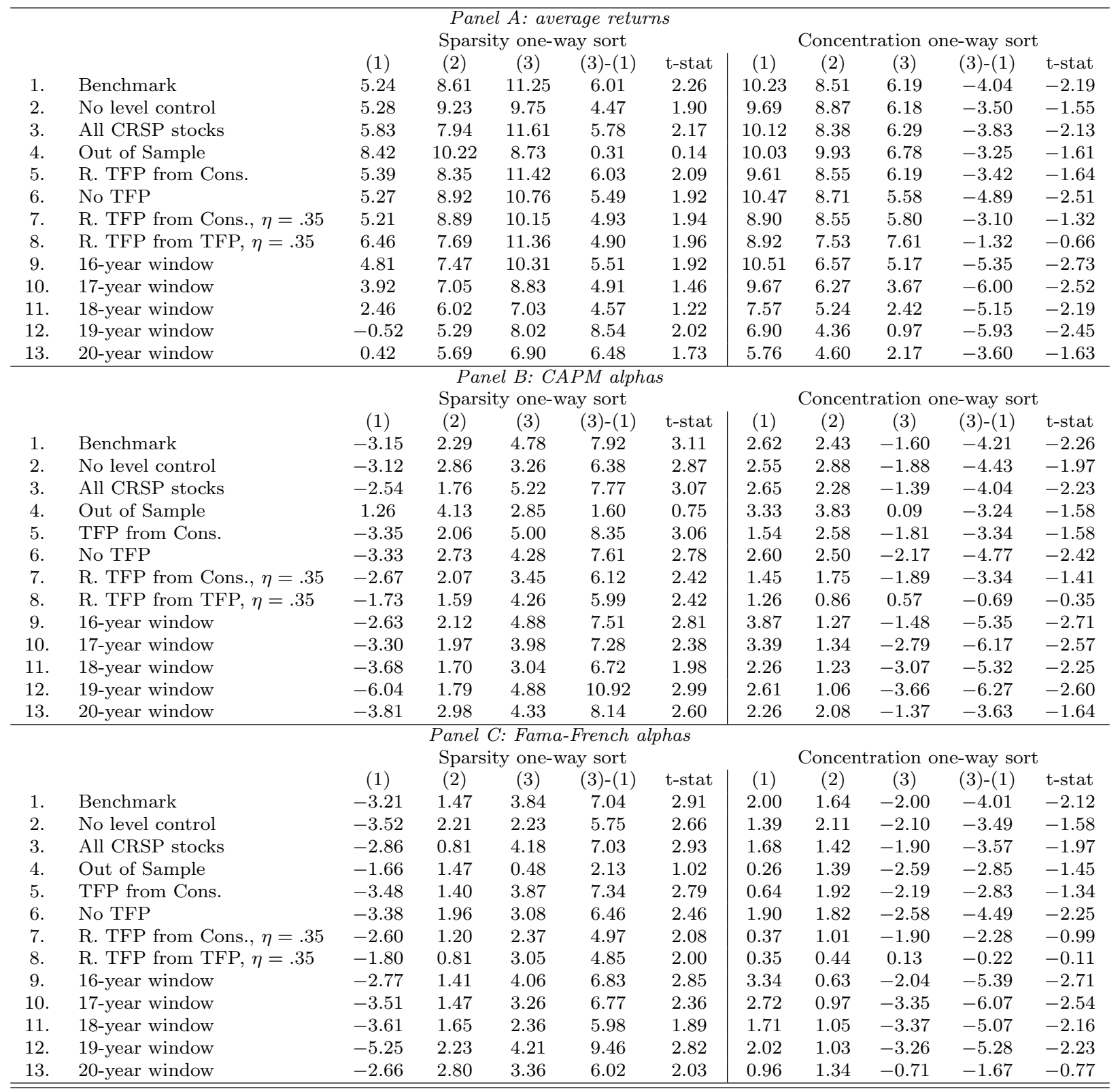


Table 8: Average $\beta$ correlations

This Table reports the time series average of the cross section correlation between the $\beta$ s estimates for all 13 specifications of Table 7

\begin{tabular}{|c|c|c|c|c|}
\hline & & Avg. $\operatorname{Corr}_{i}\left(\beta_{\mathcal{N}^{\mathcal{S}}, t}, \beta_{\mathcal{N}^{\mathcal{c}}, t}\right)$ & $\operatorname{Avg} . \operatorname{Corr}_{i}\left(\beta_{\mathcal{N}^{\mathcal{S}}, t}, \beta_{e, t}\right)$ & Avg. $\operatorname{Corr}_{i}\left(\beta_{\mathcal{N}^{c}, t}, \beta_{e, t}\right)$ \\
\hline 1. & Benchmark & -0.14 & 0.14 & -0.20 \\
\hline 2 . & No level control & -0.22 & 0.38 & -0.09 \\
\hline 3. & All CRSP stocks & -0.13 & 0.21 & -0.19 \\
\hline 4. & Out of Sample & -0.10 & 0.29 & -0.24 \\
\hline 5. & TFP from Cons. & -0.09 & 0.36 & 0.18 \\
\hline 6. & No TFP & -0.14 & - & - \\
\hline 7. & TFP from Cons., $\eta=.35$ & -0.79 & 0.78 & -0.98 \\
\hline 8. & TFP from GDP, $\eta=.35$ & -0.82 & 0.80 & -0.98 \\
\hline 9. & 16-year window & -0.14 & 0.11 & -0.16 \\
\hline 10 . & 17-year window & -0.14 & 0.08 & -0.12 \\
\hline 11. & 18-year window & -0.13 & 0.04 & -0.07 \\
\hline 12 . & 19-year window & -0.11 & -0.01 & -0.02 \\
\hline 13. & 19-year window & -0.07 & -0.09 & -0.02 \\
\hline
\end{tabular}

Table 9: Double Sort on Sparsity and Other Factors

I construct double sorted portfolios using stocks sorted on sparsity-beta and on another factor. This Table reports average excess return, post sample CAPM alphas and post sample Fama French alphas for the portfolio long on the high sparsity-beta and short on the low sparsity-beta. I consider 7 different factors for the double sort: (1) market value, (2) book to market ratio, (3) total volatility, (4) idiosyncratic volatility from CAPM model (std over 1 year of daily data), (5) idiosyncratic volatility from Fama French three-factor model (std over 1 year of daily data), (6) volume, and (7) turnover.

\begin{tabular}{llccc|cc|cc}
\hline & & \multicolumn{3}{c}{ Returns } & \multicolumn{2}{c}{$\alpha_{C A P M}$} & \multicolumn{2}{c}{$\alpha_{F F}$} \\
& & & $(3)-(1)$ & t-stat & $(3)-(1)$ & t-stat & $(3)-(1)$ & t-stat \\
\hline 1. & Market Value & $\mathrm{L}$ & 0.61 & 0.15 & 0.25 & 0.06 & 0.68 & 0.17 \\
& & $\mathrm{M}$ & 1.90 & 0.74 & 1.29 & 0.50 & 2.07 & 0.81 \\
& & $\mathrm{H}$ & 6.04 & 2.23 & 8.01 & 3.10 & 7.14 & 2.90 \\
\hline 2. & Book to Market & $\mathrm{L}$ & 5.02 & 1.66 & 6.98 & 2.39 & 5.78 & 2.07 \\
& & $\mathrm{M}$ & 4.87 & 1.58 & 6.33 & 2.07 & 6.44 & 2.16 \\
& & $\mathrm{H}$ & 1.90 & 0.45 & 4.00 & 0.95 & 3.88 & 0.92 \\
\hline 3. & Total Vol & $\mathrm{L}$ & 5.33 & 2.05 & 7.00 & 2.78 & 6.15 & 2.52 \\
& & $\mathrm{M}$ & 6.97 & 1.86 & 7.89 & 2.09 & 7.50 & 1.99 \\
& & $\mathrm{H}$ & 6.63 & 0.88 & 8.84 & 1.17 & 10.64 & 1.40 \\
\hline 4. & Idiosyncratic Vol (CAPM) & $\mathrm{L}$ & 5.59 & 2.07 & 7.39 & 2.83 & 6.37 & 2.58 \\
& & $\mathrm{M}$ & 3.70 & 0.96 & 5.18 & 1.35 & 5.69 & 1.48 \\
& & $\mathrm{H}$ & 2.95 & 0.38 & 4.68 & 0.61 & 5.96 & 0.77 \\
\hline 5. & Idiosyncratic Vol (FF) & $\mathrm{L}$ & 5.93 & 2.19 & 7.70 & 2.94 & 6.73 & 2.70 \\
& & $\mathrm{M}$ & 0.85 & 0.21 & 2.60 & 0.65 & 2.83 & 0.70 \\
& & $\mathrm{H}$ & 1.37 & 0.19 & 3.13 & 0.43 & 4.05 & 0.55 \\
\hline 6. & Volume & $\mathrm{L}$ & 0.27 & 0.09 & 0.13 & 0.04 & 0.45 & 0.15 \\
& & $\mathrm{M}$ & 3.04 & 1.30 & 2.93 & 1.23 & 3.66 & 1.58 \\
& & $\mathrm{H}$ & 5.93 & 2.17 & 7.91 & 3.03 & 7.03 & 2.83 \\
\hline 7. & Turnover & $\mathrm{L}$ & 8.74 & 2.71 & 10.43 & 3.29 & 9.48 & 3.00 \\
& & $\mathrm{M}$ & 0.97 & 0.32 & 2.74 & 0.93 & 1.50 & 0.55 \\
& & $\mathrm{H}$ & 6.74 & 1.75 & 7.56 & 1.95 & 7.44 & 1.93 \\
\hline \hline
\end{tabular}




\section{Table 10: Double Sort on Concentration and Other Factors}

I construct double sorted portfolios using stocks sorted on concentration-beta and on another factor. This Table reports average excess return, post sample CAPM alphas and post sample Fama French alphas for the portfolio long on the high concentrationbeta and short on the low concentration-beta. I consider 7 different factors for the double sort: (1) market value, (2) book to market ratio, (3) total volatility, (4) idiosyncratic volatility from CAPM model (std over 1 year of daily data), (5) idiosyncratic volatility from Fama French three-factor model (std over 1 year of daily data), (6) volume, and (7) turnover.

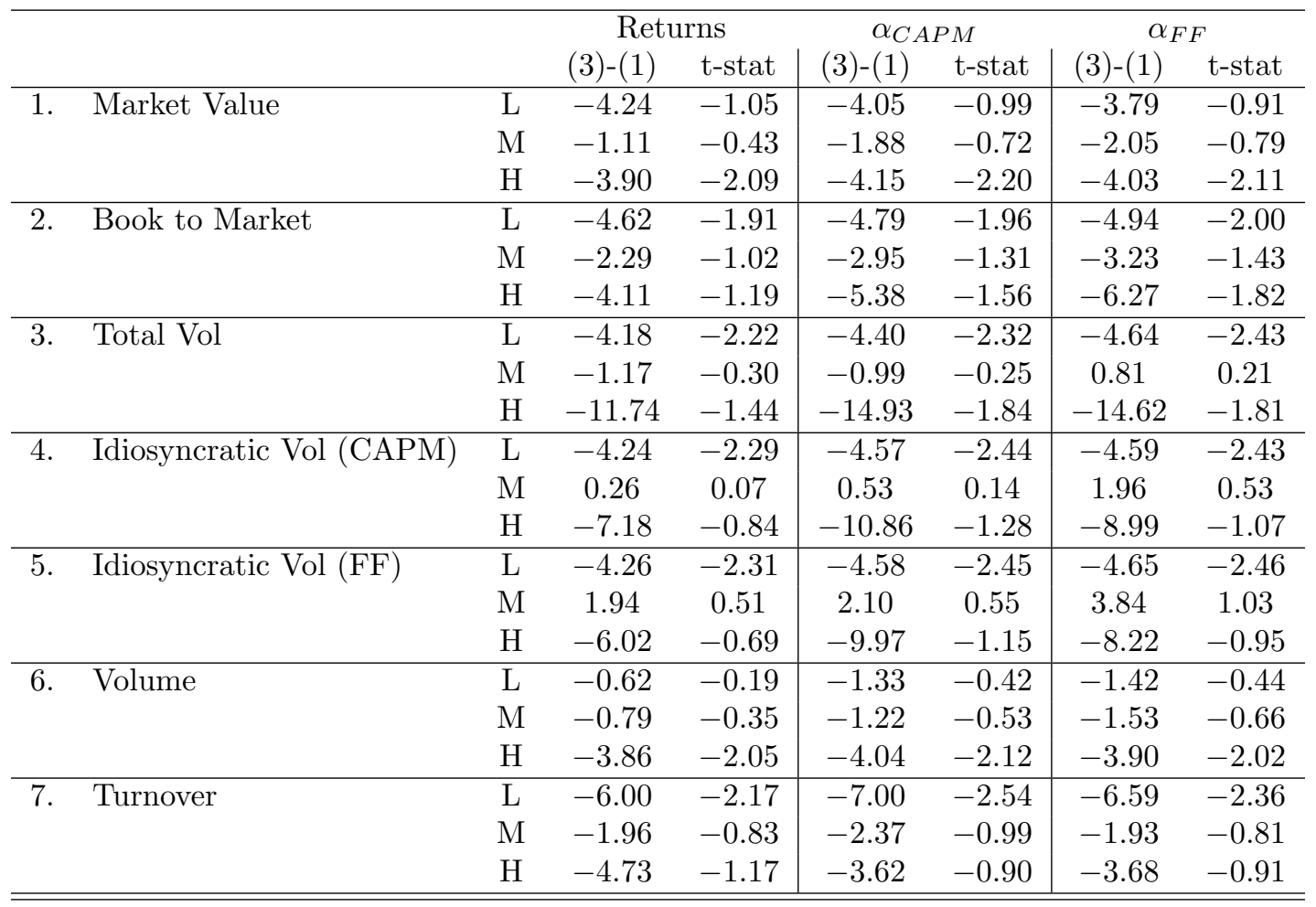

